\title{
Donor-Acceptor Triazenes: Synthesis, Characterization, and Study of Their Electronic and Thermal Properties
}

\author{
Dimitri M. Khramov and Christopher W. Bielawski* \\ Department of Chemistry and Biochemistry, University of Texas at Austin, Austin, Texas 78712 \\ bielawski@cm.utexas.edu
}

Supporting Information

\section{Table of Contents}

General considerations and additional synthetic procedures $\ldots \ldots \ldots \ldots \ldots \ldots$ S2

NMR kinetics of N-heterocyclic carbene - azide coupling reactions

S7

Tables of absolute energies, calculated dipole moments, and coordinates for

triazenes 3 (cf. Table 3 ) at B3LYP/6-31G*-level of theory

References

$\mathrm{S} 16$

NMR spectra

$\mathrm{S} 17$ 
General Considerations. All reactions were conducted under an atmosphere of nitrogen using standard Schlenk techniques or in a nitrogen filled glove-box. $\mathrm{CH}_{2} \mathrm{Cl}_{2}$ was distilled from $\mathrm{CaH}_{2}$ and degassed by two freeze-pump-thaw cycles. THF and toluene were distilled from $\mathrm{Na}$ /benzophenone and degassed by two freeze-pump-thaw cycles. 1,3-Bis(2,6-di-isopropylphenyl)imidazolium chloride was purchased from Strem Chemicals and used without further purification. 1,2-Di-iso-butylaminobenzene, ${ }^{1}$ 1,2-di-neo-pentylaminobenzene, ${ }^{2}$ and 1,3-di-methyl-benzimidazolium iodide ${ }^{3}$ were prepared as previously described. All other reagents were used without further purification, unless otherwise noted. ${ }^{1} \mathrm{H}$ NMR spectra were recorded using a $300 \mathrm{MHz}$ or $400 \mathrm{MHz}$ spectrometer. Chemical shifts are reported in delta $(\delta)$ units, expressed in parts per million (ppm) downfield from tetramethylsilane using residual protonated solvent as an internal standard $\left(\mathrm{CDCl}_{3}, 7.24 \mathrm{ppm} ; \mathrm{C}_{6} \mathrm{D}_{6}, 7.15 \mathrm{ppm} ; \mathrm{CD}_{2} \mathrm{Cl}_{2}, 5.32\right.$ ppm; DMSO-d $6,2.49$ ppm). ${ }^{13} \mathrm{C}$ NMR spectra were recorded using a $75 \mathrm{MHz}$ or $100 \mathrm{MHz}$ spectrometer. Chemical shifts are reported in delta $(\delta)$ units, expressed in parts per million (ppm) downfield from tetramethylsilane using the solvent as an internal standard $\left(\mathrm{CDCl}_{3}, 77.0 \mathrm{ppm} ; \mathrm{C}_{6} \mathrm{D}_{6}\right.$, 128.0 ppm; $\left.\mathrm{CD}_{2} \mathrm{Cl}_{2}, 53.8 \mathrm{ppm} ; \mathrm{DMSO}-d_{6}, 39.5 \mathrm{ppm}\right) .{ }^{13} \mathrm{C} \mathrm{NMR}$ spectra were routinely run with broadband decoupling. High-resolution mass spectra (HRMS) are reported as m/z (relative intensity). Melting points were determined under ambient atmosphere and are uncorrected. Decomposition temperatures $\left(\mathrm{T}_{\mathrm{d}} \mathrm{s}\right)$ were determined under nitrogen atmosphere and were reported as the temperature at which 5\% weight loss occurred using a proprietary high resolution dynamic heating method. X-ray

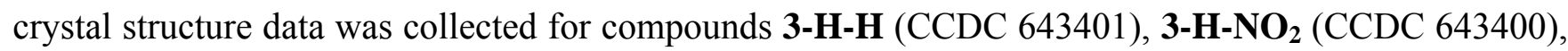
3-OCH $\mathbf{- H}\left(\mathrm{CCDC}\right.$ 643398) and 3-OCH$-\mathbf{O C O}_{2}(\mathrm{CCDC}$ 643399), and deposited with the Cambridge Crystallographic Data Centre, 12 Union Road, Cambridge CB2 1EZ, UK.

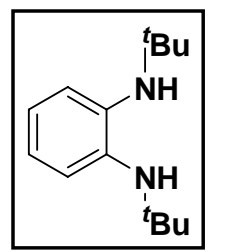

1,2-Di-tert-butylaminobenzene (A). Following a previously reported procedure ${ }^{4}{ }^{1,2-}$ dibromobenzene $(1.12 \mathrm{~g}, 4.75 \mathrm{mmol})$ was coupled to tert-butylamine $(0.73 \mathrm{~g}, 10 \mathrm{mmol})$ to afford $1.04 \mathrm{~g}$ (99\% yield) of the titled compound as a brown oil. ${ }^{1} \mathrm{H} \mathrm{NMR}\left(\mathrm{CDCl}_{3}\right): \delta 6.82$ $(\mathrm{dd}, \mathrm{J}=3.6,11.6 \mathrm{~Hz}, 2 \mathrm{H}), 6.75(\mathrm{dd}, \mathrm{J}=3.6,11.6 \mathrm{~Hz}, 2 \mathrm{H}), 3.7(\mathrm{br}, 2 \mathrm{H}), 1.28(\mathrm{~s}, 18 \mathrm{H}) .{ }^{13} \mathrm{C} \mathrm{NMR}$ 
$\left(\mathrm{CDCl}_{3}\right): \delta 138.5,120.5,120.1,51.9,29.9$. HRMS: $[\mathrm{M}+\mathrm{H}]^{+}$calcd for $\mathrm{C}_{14} \mathrm{H}_{25} \mathrm{~N}_{2}: 221.2018$; found, 221.2014

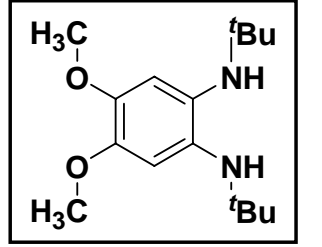

1,2-Di-tert-butylamino-4,5-dimethoxybenzene (B). Following a previously reported procedure $,{ }^{4} 4,5$-dibromoveratrole $(1.48 \mathrm{~g}, 5.0 \mathrm{mmol})$ was coupled to tertbutylamine $(0.80 \mathrm{~g}, 11 \mathrm{mmol})$ to afford $1.4 \mathrm{~g}$ (99\% yield) of the titled compound as a brown oil. ${ }^{1} \mathrm{H}$ NMR $\left(\mathrm{CDCl}_{3}\right): \delta 6.55(\mathrm{~s}, 2 \mathrm{H}), 3.78(\mathrm{~s}, 6 \mathrm{H}), 3.41(\mathrm{br}, 2 \mathrm{H}), 1.20(\mathrm{~s}, 18 \mathrm{H}) .{ }^{13} \mathrm{C}$ NMR $\left(\mathrm{CDCl}_{3}\right): \delta 143.4,132.6,108.0,56.3,52.4,30.1$. HRMS: $[\mathrm{M}+\mathrm{H}]^{+}$calcd for $\mathrm{C}_{16} \mathrm{H}_{29} \mathrm{~N}_{2} \mathrm{O}_{2}:$ 281.2229; found, 281.2229 .

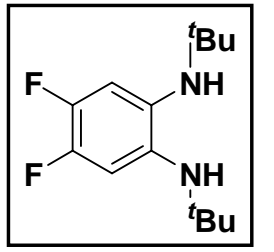

1,2-Di-tert-butylamino-4,5-difluorobenzene (C). Following a previously reported procedure ${ }^{4}{ }^{1,2}$-difluoro-4,5-dibromobenzene $(1.36 \mathrm{~g}, 5.0 \mathrm{mmol})$ was coupled to tertbutylamine $(0.80 \mathrm{~g}, 11 \mathrm{mmol})$ to afford $1.4 \mathrm{~g}(99 \%$ yield $)$ of the titled compound as a brown oil, which was used without additional purification. ${ }^{1} \mathrm{H} \mathrm{NMR}\left(\mathrm{CDCl}_{3}\right): \delta 6.67\left(\mathrm{t}, \mathrm{J}_{\mathrm{F}-\mathrm{H}}=10.4 \mathrm{~Hz}\right.$, 2H), $3.55(\mathrm{br}, 2 \mathrm{H}), 1.23(\mathrm{~s}, 18 \mathrm{H}) .{ }^{13} \mathrm{C} \mathrm{NMR}\left(\mathrm{CDCl}_{3}\right): \delta 144.1\left(\mathrm{dd}, \mathrm{J}_{\mathrm{C}-\mathrm{F}}=14.6,240 \mathrm{~Hz}\right), 134.6(\mathrm{~m})$, $108.9(\mathrm{~m})$, 52.0, 29.7. HRMS: $[\mathrm{M}+\mathrm{H}]^{+}$calcd for $\mathrm{C}_{14} \mathrm{H}_{23} \mathrm{~F}_{2} \mathrm{~N}_{2}$ : 257.1829; found, 257.1826.

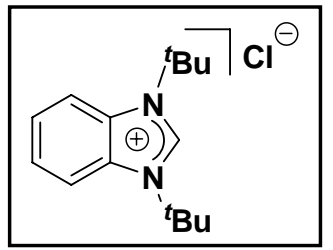

1,3-Di-tert-butyl-benzimidazolium chloride (D). Crude 1,2-di-tertbutylaminobenzene (A) $(1.10 \mathrm{~g}, 5 \mathrm{mmol})$ was treated with aq. $\mathrm{HCl}(5.1 \mathrm{mmol}, 0.47$ $\mathrm{mL})$ in $20 \mathrm{~mL}$ of $\mathrm{HC}(\mathrm{OEt})_{3}$. The resulting reaction mixture was stirred for $10 \mathrm{~h} \mathrm{at}$ $50{ }^{\circ} \mathrm{C}$ and then concentrated to one-half of its original volume, which caused solids to precipitate. These solids were then collected by filtration and washed with diethyl ether $(20 \mathrm{~mL})$ to afford $1.2 \mathrm{~g}$ of the titled compound as a tan powder (90\% yield). ${ }^{1} \mathrm{H}$ NMR (DMSO-d6): $\delta 8.93(\mathrm{~s}, 1 \mathrm{H}), 8.32(\mathrm{dd}, \mathrm{J}=$ 3.2, $6.2 \mathrm{~Hz}, 2 \mathrm{H}), 7.63(\mathrm{dd}, \mathrm{J}=3.2,6.2 \mathrm{~Hz}, 2 \mathrm{H}), 1.82(\mathrm{~s}, 18 \mathrm{H}) .{ }^{13} \mathrm{C}$ NMR (DMSO-d 6$): \delta 139.1,130.4$, 125.7, 116.6, 60.9, 28.0. HRMS: [M] $]^{+}$calcd for $\mathrm{C}_{15} \mathrm{H}_{23} \mathrm{~N}_{2}$ : 231.1861; found, 231.1860. 


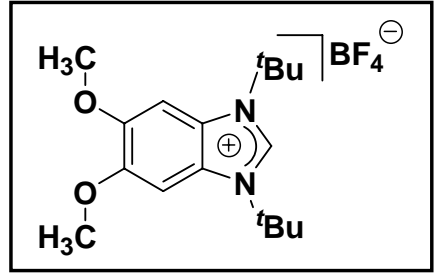

1,3-Di-tert-butyl-4,5-dimethoxy-benzimidazolium tetrafluoroborate (E).

Crude 1,2-di-tert-butylamino-4,5-dimethoxybenzene (B) $(1.40 \mathrm{~g}, 5 \mathrm{mmol})$ was treated with aq. $\mathrm{HBF}_{4}(5.1 \mathrm{mmol}, 0.65 \mathrm{~mL})$ in $20 \mathrm{~mL}$ of $\mathrm{HC}(\mathrm{OEt})_{3}$. The

resulting reaction mixture was stirred for $10 \mathrm{~h}$ at $50{ }^{\circ} \mathrm{C}$ and then concentrated to one-half of its original volume, which caused solids to precipitate. These solids were then collected by filtration and washed with diethyl ether $(20 \mathrm{~mL})$ to afford $1.8 \mathrm{~g}$ of the titled compound as a white powder ( $94 \%$ yield). ${ }^{1} \mathrm{H}$ NMR (DMSO-d $\left.d_{6}\right): \delta 8.66(\mathrm{~s}, 1 \mathrm{H}), 7.48(\mathrm{~s}, 2 \mathrm{H}), 3.96(\mathrm{~s}, 6 \mathrm{H}), 1.81(\mathrm{~s}, 18 \mathrm{H}),{ }^{13} \mathrm{C}$ NMR (DMSO-d 6 ): $\delta$ 148.6, 136.1, 124.9, 98.0, 60.6, 56.6, 28.1. HRMS: [M] $]^{+}$calcd for $\mathrm{C}_{17} \mathrm{H}_{27} \mathrm{~N}_{2} \mathrm{O}_{2}$ : 291.2067; found, 291.2073.

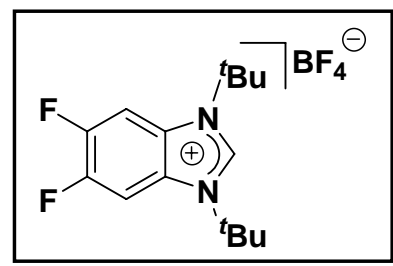

\section{1,3-Di-tert-butyl-4,5-difluoro-benzimidazolium tetrafluoroborate (F). Crude 1,2-di-tert-butylamino-4,5-difluorobenzene (C) (1.3 g, $5 \mathrm{mmol})$ was treated with a $6.8 \mathrm{M}$ diethyl ether solution of $\mathrm{HBF}_{4}(5.1 \mathrm{mmol}, 0.75 \mathrm{~mL})$ in 20}

$\mathrm{mL}$ of $\mathrm{HC}(\mathrm{OEt})_{3}$. The resulting reaction mixture was stirred for $10 \mathrm{~h}$ at $50{ }^{\circ} \mathrm{C}$ and then concentrated to one-half of its original volume, which caused solids to precipitate. These solids was then collected by filtration and washed with diethyl ether $(20 \mathrm{~mL})$ to afford $1.56 \mathrm{~g}$ of the titled compound as a grey powder (87\% yield). ${ }^{1} \mathrm{H}$ NMR (DMSO-d 6 ): $\delta 8.87(\mathrm{~s}, 1 \mathrm{H}), 8.62\left(\mathrm{t}, \mathrm{J}_{\mathrm{H}-\mathrm{F}}=9.2 \mathrm{~Hz}, 2 \mathrm{H}\right), 1.79(\mathrm{~s}, 18 \mathrm{H})$. ${ }^{13} \mathrm{C}$ NMR (DMSO-d6): $\delta 148.3\left(\mathrm{~d}, \mathrm{~J}_{\mathrm{C}-\mathrm{F}}=249 \mathrm{~Hz}\right), 140.6,126.8\left(\mathrm{t}, \mathrm{J}_{\mathrm{C}-\mathrm{F}}=6.1 \mathrm{~Hz}\right), 105.3(\mathrm{~m}), 61.6,27.8$. HRMS: $[\mathrm{M}]^{+}$calcd for $\mathrm{C}_{15} \mathrm{H}_{21} \mathrm{~F}_{2} \mathrm{~N}_{2}: 267.1673$; found, 267.1676.

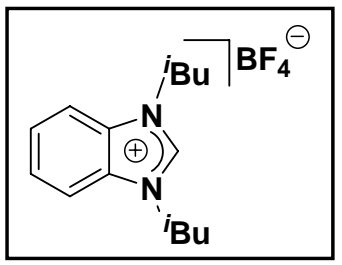

\section{1,3-Di-iso-butyl-benzimidazolium tetrafluoroborate (G). 1,2-Di-iso-} butylaminobenzene $\mathrm{e}^{1}(5.14 \mathrm{~g}, 23.4 \mathrm{mmol})$ was treated with aq. $\mathrm{HBF}_{4}(25 \mathrm{mmol}, 3.2$ $\mathrm{mL})$ in $50 \mathrm{~mL} \mathrm{HC}(\mathrm{OEt})_{3}$. The resulting reaction mixture was stirred at $60{ }^{\circ} \mathrm{C}$ for

$10 \mathrm{~h}$ and then concentrated to one-half of its original volume, which caused solids to precipitate. After washing the solids with diethyl ether $(30 \mathrm{~mL})$, these solids were dried under high vacuum to give $3.13 \mathrm{~g}$ 
of the titled compound as a light pink solid (42\% yield). Spectral data were in accord with literature reports. $^{5}$

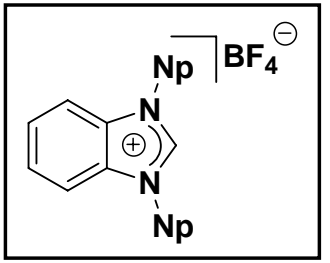

1,3-Di-neo-pentyl-benzimidazolium tetrafluoroborate $\mathbf{( H )}$, 1,2-Di-neopentylaminobenzene ${ }^{2}(3.5 \mathrm{~g}, 14.1 \mathrm{mmol})$ was treated with aq. $\mathrm{HBF}_{4}(16 \mathrm{mmol}, 2.1$ $\mathrm{mL})$ in $30 \mathrm{~mL} \mathrm{HC}(\mathrm{OEt})_{3}$. The resulting reaction mixture was stirred at $60{ }^{\circ} \mathrm{C}$ for $10 \mathrm{~h}$ and then concentrated to one-half of its original volume, which caused solids to precipitate. After washing the solids with diethyl ether $(50 \mathrm{~mL})$, they were dried under high vacuum to give $4.3 \mathrm{~g}$ of the titled compound as a white solid (88\% yield). ${ }^{1} \mathrm{H}$ NMR (DMSO- $\left.d_{6}\right): \delta 9.68(\mathrm{~s}, 1 \mathrm{H}), 8.16(\mathrm{dd}, \mathrm{J}=3.0$, 6.4, Hz, 2H), $7.68(\mathrm{dd}, \mathrm{J}=3.0,6.4 \mathrm{~Hz}, 2 \mathrm{H}), 4.39(\mathrm{~s}, 4 \mathrm{H}), 0.99$ (s, 18H). ${ }^{13} \mathrm{C}$ NMR (DMSO-d6): $\delta$ 143.6, 132.0, 126.4, 114.2, 56.8, 33.1, 27.0. HRMS: $[\mathrm{M}]^{+}$calcd for $\mathrm{C}_{17} \mathrm{H}_{27} \mathrm{~N}_{2}$ : 259.2174; found, 259.2142 .

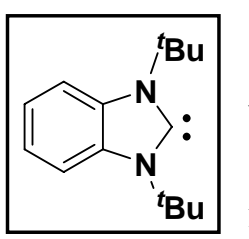

1,3-Di-tert-butyl-benzimidazol-2-ylidene (I). A mixture of 1,3-di-tert-butylbenzimidazolium chloride (D) (1.55 g, $5.8 \mathrm{mmol})$, potassium tert-butoxide $(0.65 \mathrm{~g}, 5.8$ $\mathrm{mmol}$ ), and $15 \mathrm{~mL}$ THF were stirred at ambient temperature for $2 \mathrm{~h}$. After removing inorganic salts which precipitated from the reaction mixture by filtration, the resulting solution was concentrated to afford $1.3 \mathrm{~g}$ of the titled compound as a brown powder $(97 \%$ yield $) .{ }^{1} \mathrm{H}$ NMR $\left(\mathrm{C}_{6} \mathrm{D}_{6}\right): \delta$ $7.41(\mathrm{dd}, \mathrm{J}=3.6,6.2 \mathrm{~Hz}, 2 \mathrm{H}), 7.00(\mathrm{dd}, \mathrm{J}=3.6,6.2 \mathrm{~Hz}, 2 \mathrm{H}), 1.69(\mathrm{~s}, 18 \mathrm{H}) .{ }^{13} \mathrm{C}$ NMR $\left(\mathrm{C}_{6} \mathrm{D}_{6}\right): \delta 224.7$, 135.6, 120.2, 113.9, 57.3, 30.5. HRMS: $[\mathrm{M}+\mathrm{H}]^{+}$calcd for $\mathrm{C}_{15} \mathrm{H}_{23} \mathrm{~N}_{2}$ : 231.1861; found, 231.1865.

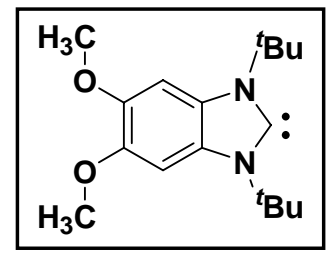

1,3-Di-tert-butyl-4,5-dimethoxy-benzimidazol-2-ylidene (J). A mixture of 1,3-ditert-butyl-4,5-dimethoxy-benzimidazolium tetrafluoroborate $(\mathbf{E})$ (1.13 g, $3 \mathrm{mmol})$, potassium tert-butoxide $(0.34 \mathrm{~g}, 3 \mathrm{mmol})$, and $17 \mathrm{~mL}$ THF were stirred at ambient temperature for $2 \mathrm{~h}$. After removing inorganic salts which precipitated from the reaction mixture by filtration, the resulting solution was concentrated to afford $0.83 \mathrm{~g}$ of the titled compound as a white 
powder (95\% yield). ${ }^{1} \mathrm{H}$ NMR $\left(\mathrm{C}_{6} \mathrm{D}_{6}\right): \delta 7.12(\mathrm{~s}, 2 \mathrm{H}), 3.52(\mathrm{~s}, 6 \mathrm{H}), 1.74(\mathrm{~s}, 18 \mathrm{H}) .{ }^{13} \mathrm{C}$ NMR $\left(\mathrm{C}_{6} \mathrm{D}_{6}\right): \delta$ 223.1, 145.7, 129.8, 99.8, 57.0, 56.8, 30.6. HRMS: $[\mathrm{M}+\mathrm{H}]^{+}$calcd for $\mathrm{C}_{17} \mathrm{H}_{27} \mathrm{~N}_{2} \mathrm{O}_{2}$ : 291.2067; found, 291.2073.

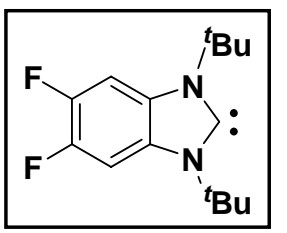

1,3-Di-tert-butyl-4,5-difluoro-benzimidazol-2-ylidene (K). A mixture of 1,3-di-tertbutyl-4,5-difluoro-benzimidazolium tetrafluoroborate (F) (1.0 $\mathrm{g}$, $2.83 \mathrm{mmol})$, potassium tert-butoxide $(0.32 \mathrm{~g}, 2.83 \mathrm{mmol})$, and $10 \mathrm{~mL}$ THF were stirred at ambient temperature for $2 \mathrm{~h}$. After removing inorganic salts which precipitated from the reaction mixture by filtration, the resulting solution was concentrated to afford $0.72 \mathrm{~g}$ of the titled compound as a brown powder $\left(96 \%\right.$ yield). ${ }^{1} \mathrm{H}$ NMR $\left(\mathrm{C}_{6} \mathrm{D}_{6}\right): \delta 7.16\left(\mathrm{t}, \mathrm{J}_{\mathrm{F}-\mathrm{C}}=8.8 \mathrm{~Hz}, 2 \mathrm{H}\right), 1.51(\mathrm{~s}, 18 \mathrm{H}) .{ }^{13} \mathrm{C}$ NMR $\left(\mathrm{C}_{6} \mathrm{D}_{6}\right): \delta$ 228.5, $145.8\left(\mathrm{dd}, \mathrm{J}_{\mathrm{F}-\mathrm{C}}=17,234 \mathrm{~Hz}\right), 130.6\left(\mathrm{t}, \mathrm{J}_{\mathrm{F}-\mathrm{C}}=4.1 \mathrm{~Hz}\right), 50.8\left(\mathrm{dd}, \mathrm{J}_{\mathrm{F}-\mathrm{C}}=8.8,14.3 \mathrm{~Hz}\right), 57.5,30.3$. HRMS: $[\mathrm{M}+\mathrm{H}]^{+}$calcd for $\mathrm{C}_{15} \mathrm{H}_{21} \mathrm{~F}_{2} \mathrm{~N}_{2}: 267.1673$; found, 267.1677.

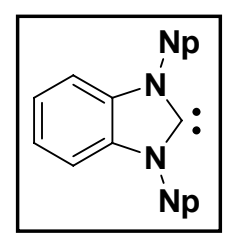

1,3-Di-neo-pentyl-benzimidazol-2-ylidene (L). A mixture of 1,3-di-neo-pentylbenzimidazolium tetrafluoroborate $(\mathbf{H})(1.0 \mathrm{~g}, 2.89 \mathrm{mmol})$, potassium tert-butoxide $(0.32$ $\mathrm{g}, 2.89 \mathrm{mmol}$ ), and $10 \mathrm{~mL}$ THF were stirred at ambient temperature for $30 \mathrm{~min}$. After removing inorganic salts which precipitated from the reaction mixture by filtration, the resulting solution was concentrated to afford $0.64 \mathrm{~g}$ of the titled compound ( $96 \%$ yield) as a light brown solid. Spectral data were in accord with literature reports. ${ }^{6}$ 
NMR Kinetics of N-Heterocyclic Carbene - Azide Coupling Reactions. Three separate kinetics experiments were performed by independently studying the rate of reaction between a free Nheterocyclic carbene (NHC), 1,3-di-tert-butyl-benzimidazol-2-ylidene, and three different azides: phenyl azide, para-methoxyphenyl azide, and para-nitrophenyl azide. In these experiments, stock solutions of the NHC and various azides were first prepared and utilized. A vial containing 1,3-di-tertbutyl-benzimidazol-2-ylidene $(115 \mathrm{mg}, 0.50 \mathrm{mmol})$ was dissolved in $2.5 \mathrm{~mL}$ of THF- $d_{8}$ to create a 0.2 M stock solution of this compound. Three separate vials were then independently charged with phenyl azide (30 mg, $0.25 \mathrm{mmol}$ ), para-methoxyphenyl azide (38 $\mathrm{mg}, 0.25 \mathrm{mmol}$ ), and para-nitrophenyl azide (41 mg, $0.25 \mathrm{mmol}$ ), respectively. To each vial containing an azide, $1.25 \mathrm{~mL}$ of THF- $d_{8}$ was added to create $0.2 \mathrm{M}$ stock solutions of these compounds. Three separate NMR tubes were then independently charged with $1 \mathrm{~mL}$ of THF- $d_{8}$ and $0.3 \mathrm{~mL}(0.06 \mathrm{mmol})$ of the NHC stock solution. After recording an initial spectrum, $0.3 \mathrm{~mL}(0.06 \mathrm{mmol})$ of an organic azide stock solution was added to generate solutions with initial substrate concentrations of $37.5 \mathrm{mM}\left([\mathrm{NHC}]_{0}=[\text { azide }]_{0}=[\text { substrate }]_{0}\right)$. Using ${ }^{1} \mathrm{H}$ NMR spectroscopy, the coupling reactions were monitored over time by integrating peak areas of diagnostic signals attributed to starting materials relative to analogous signals attributed to their respective triazene products. To determine rate constants $(k)$, the data was fitted to Eq. 1, the integrated rate law of the bimolecular reaction: $2 \cdot[$ substrate $] \rightarrow$ [product $]^{7}$

$$
1 /[\text { substrate }]=k t+1 /[\text { substrate }]_{0}(1)
$$

The observed rate constants are summarized in Table S1; experimental data (with a linear fit corresponding to Eq. 1) are shown in Figures S1 - S3. 
Table S1. Rate constants for a variety of triazene forming reactions.

Entry

${ }^{\mathrm{a}}$ General reaction conditions: $[\mathrm{NHC}]_{0}=[\text { azide }]_{0}=37.5 \mathrm{mM}$, solvent $=$ THF- $d_{8}$, temperature $=23{ }^{\circ} \mathrm{C}$.

${ }^{\mathrm{b}}$ Rate constants $(k)$ were determined by fitting the concentrations of the substrates, as determined by integrating the areas of diagnostic ${ }^{1} \mathrm{H}$ NMR signals, to Eq. 1 as a function of time (see Figures $\mathrm{S} 1$ - S3 for experimental data).

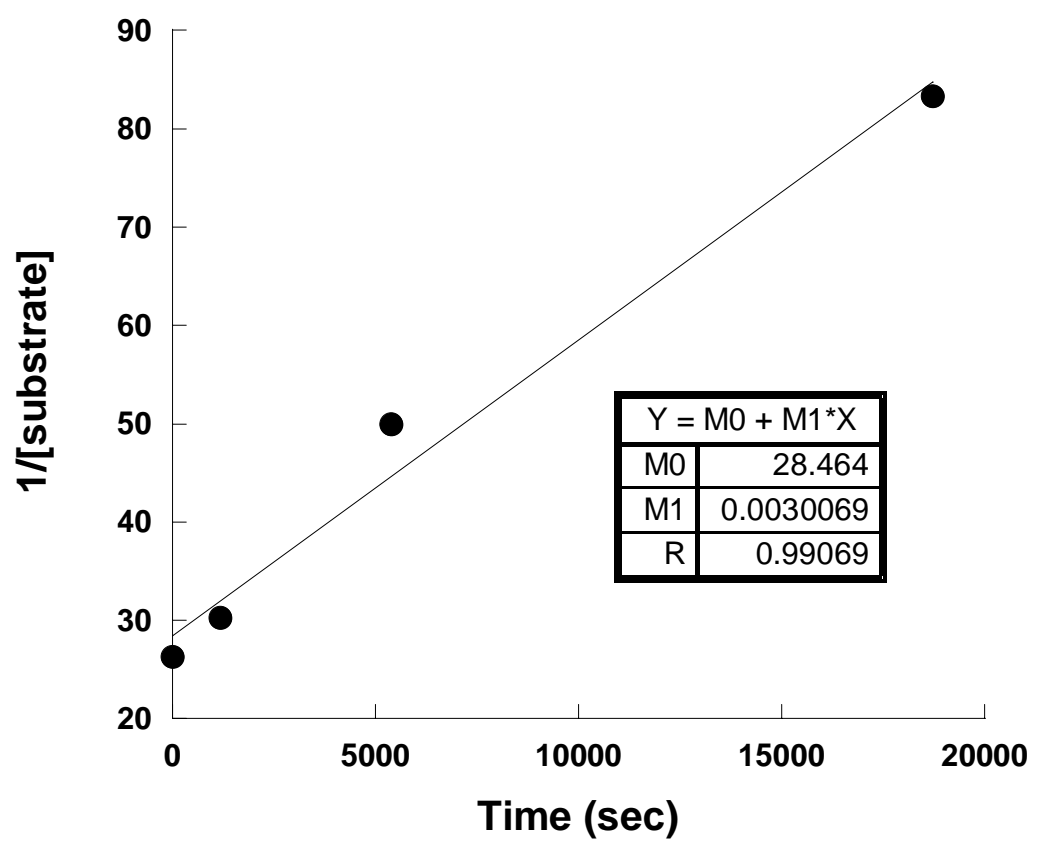

Figure S1. Plot of 1/[substrate] versus time for the reaction of 1,3-di-tert-butylbenzimidazol-2-ylidene with phenylazide (Table S1, Entry 1). 


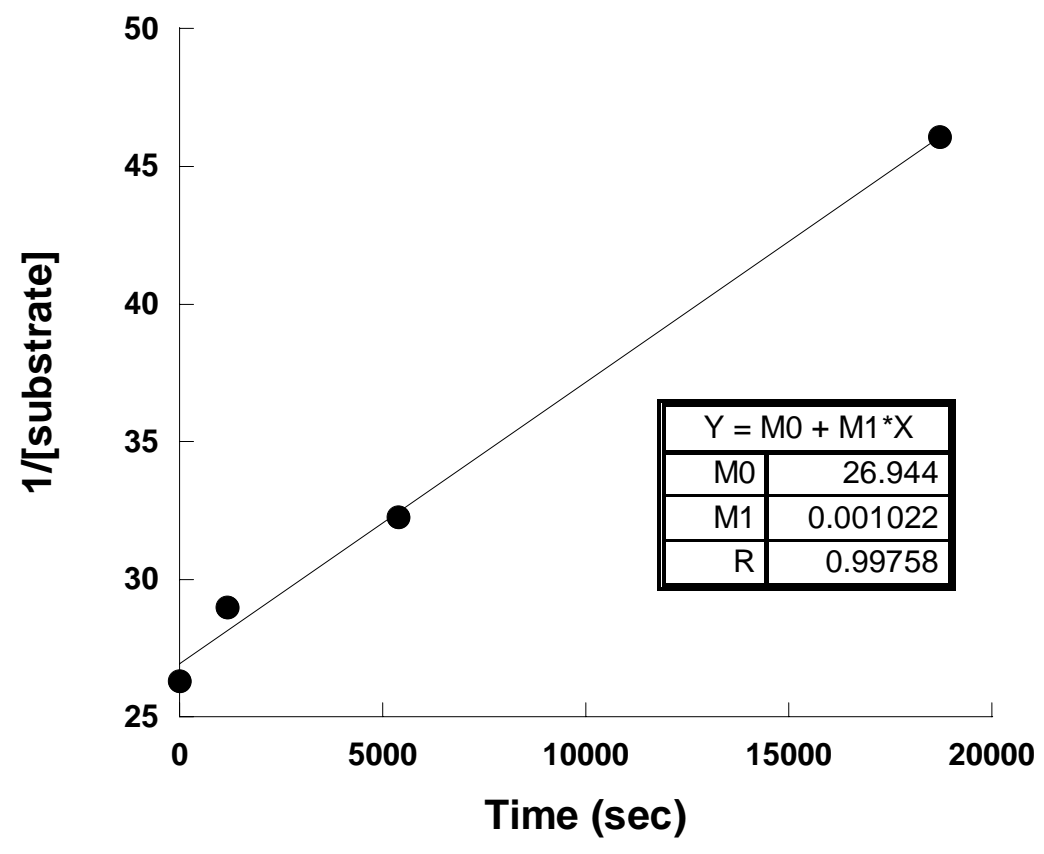

Figure S2. Plot of 1/[substrate] versus time for the reaction of 1,3-di-tert-butylbenzimidazol-2-ylidene with para-methoxyphenyl azide (Table S1, Entry 2).

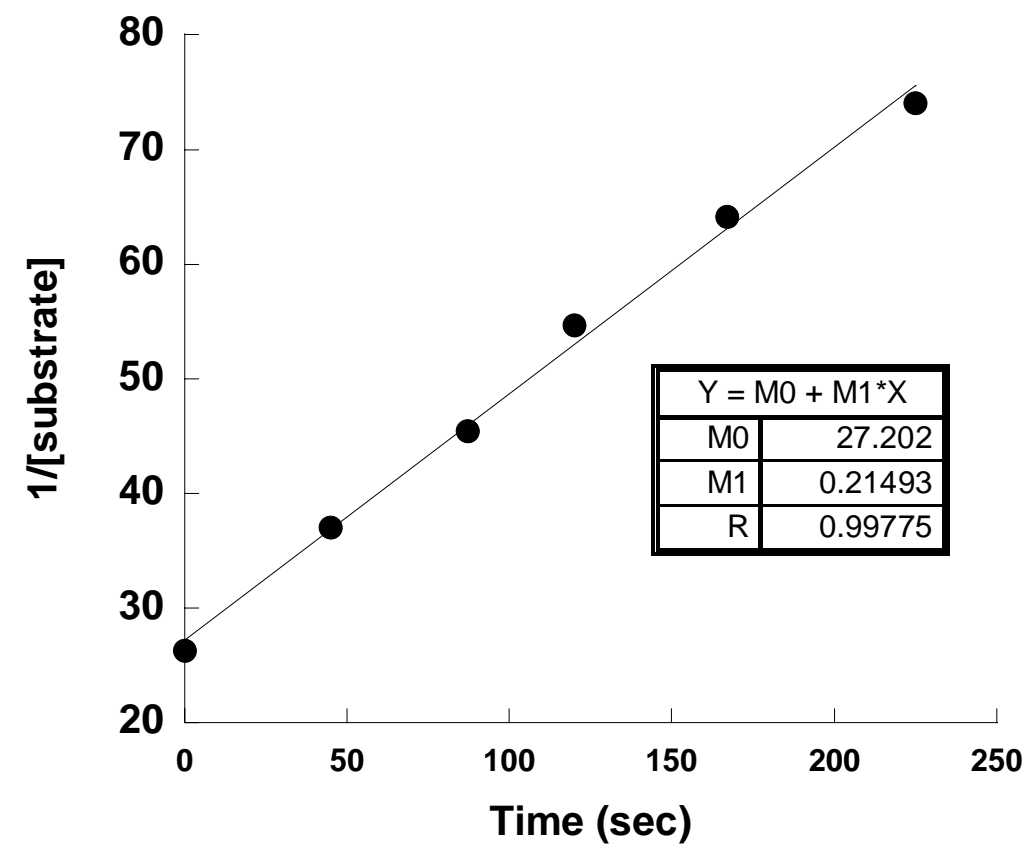

Figure S3. Plot of 1/[substrate] versus time for the reaction of 1,3-di-tert-butylbenzimidazol-2-ylidene with para-nitrophenyl azide (Table S1, Entry 3). 
Table S2. Absolute energy, calculated dipole moment, and coordinates for triazene 3-H-H (cf. Table 3, Entry 1) at B3LYP/6-31G*-level of theory. ${ }^{8}$

$E(H F)=-854.3416596 \mathrm{a} \cdot \mathrm{u}$.

$\mu_{\text {calc }}=4.29$ debye

\begin{tabular}{rll}
\multicolumn{3}{c}{ Atom } \\
\hline 1 & $-\cdot-\cdot-$ \\
2 & $\mathrm{H}$ & $\mathrm{H} 1$ \\
3 & $\mathrm{C}$ & $\mathrm{C} 1$ \\
3 & $\mathrm{C}$ & $\mathrm{C} 4$ \\
4 & $\mathrm{C}$ & $\mathrm{C} 2$ \\
5 & $\mathrm{C}$ & $\mathrm{C} 6$ \\
6 & $\mathrm{C}$ & $\mathrm{C} 5$ \\
7 & $\mathrm{C}$ & $\mathrm{C} 3$ \\
8 & $\mathrm{H}$ & $\mathrm{H} 6$ \\
9 & $\mathrm{H}$ & $\mathrm{H} 5$ \\
10 & $\mathrm{H}$ & $\mathrm{H} 4$ \\
11 & $\mathrm{~N}$ & $\mathrm{~N} 1$ \\
12 & $\mathrm{~N}$ & $\mathrm{~N} 2$ \\
13 & $\mathrm{C}$ & $\mathrm{C} 7$ \\
14 & $\mathrm{C}$ & $\mathrm{C} 8$ \\
15 & $\mathrm{H}$ & $\mathrm{H} 7$ \\
16 & $\mathrm{H}$ & $\mathrm{H} 9$ \\
17 & $\mathrm{H}$ & $\mathrm{H} 10$ \\
18 & $\mathrm{C}$ & $\mathrm{C} 9$ \\
19 & $\mathrm{H}$ & $\mathrm{H} 8$ \\
20 & $\mathrm{H}$ & $\mathrm{H} 11$ \\
21 & $\mathrm{H}$ & $\mathrm{H} 12$ \\
22 & $\mathrm{~N}$ & $\mathrm{~N} 3$ \\
23 & $\mathrm{~N}$ & $\mathrm{~N} 4$ \\
24 & $\mathrm{~N}$ & $\mathrm{~N} 5$ \\
25 & $\mathrm{C}$ & $\mathrm{C} 10$ \\
26 & $\mathrm{C}$ & $\mathrm{C} 11$ \\
27 & $\mathrm{C}$ & $\mathrm{C} 12$ \\
28 & $\mathrm{C}$ & $\mathrm{C} 13$ \\
29 & $\mathrm{C}$ & $\mathrm{C} 14$ \\
30 & $\mathrm{C}$ & $\mathrm{C} 15$ \\
31 & $\mathrm{H}$ & $\mathrm{H} 3$ \\
32 & $\mathrm{H}$ & $\mathrm{H} 13$ \\
33 & $\mathrm{H}$ & $\mathrm{H} 14$ \\
34 & $\mathrm{H}$ & $\mathrm{H} 15$ \\
35 & $\mathrm{H}$ & $\mathrm{H} 16$ \\
& & \\
13 &
\end{tabular}

$\underset{\mathrm{X}}{\operatorname{Cartesian}} \underset{\mathrm{Y}}{\mathrm{C}} \mathrm{Z}$

\begin{tabular}{|c|c|c|}
\hline 0465456 & $\ldots$ & \\
\hline . 0465456 & -2.9404470 & -0 \\
\hline .4724851 & -1.9477675 & $-\odot$ \\
\hline-4.6045964 & 0. 6537080 & 595 \\
\hline-2.6708728 & - ๑. 8142976 & -0.1058249 \\
\hline-4.8584759 & -1.7634029 & -0.079341 \\
\hline - 3.2294208 & $\odot .4673606$ & $\odot .042347$ \\
\hline - 5.5108982 & -2.6303626 & -0.121977 \\
\hline - 6.4915337 & -0.3767028 & 0.133899 \\
\hline-5.0361664 & 1.6429421 & 0.243606 \\
\hline-1.2848699 & $-\odot .6596482$ & -0.170133 \\
\hline-2.1729539 & 1.3636700 & ๑.076659 \\
\hline - ०. 9709028 & ○. 6846481 & -0.03 \\
\hline-0.3764244 & -1.7765217 & -0.395180 \\
\hline ๑. 5719631 & -1.4001808 & -0.768056 \\
\hline-0.8303430 & -2.4537118 & -1.125752 \\
\hline-0.1931244 & -2.3246233 & 0.536 \\
\hline-2.2815587 & 2.8022014 & 0.238519 \\
\hline - 2.8725008 & 3.2312743 & -0.578239 \\
\hline-1.2722016 & 3. 2125142 & ๑. 217788 \\
\hline-2.7607001 & 3.0432228 & 1.194129 \\
\hline ๑. 1531270 & 1.3666611 & -0.029262 \\
\hline 1.2867787 & 0.6174254 & 0.020678 \\
\hline 2.3183361 & 1.3421372 & -0.110202 \\
\hline 3.5329709 & ○. 6242634 & ๑. . 001924 \\
\hline 6.0611299 & - ๑. 5891295 & 0.145165 \\
\hline 3.6752438 & $-\odot .6560523$ & $\odot .567501$ \\
\hline 4.6740482 & 1.2926145 & -0.466798 \\
\hline 5.9272246 & ๑. 6875849 & -0.404074 \\
\hline 4.9307927 & -1.2527717 & 0.633607 \\
\hline 2.7996926 & -1.1597746 & 0.962383 \\
\hline 4.5465914 & 2.2873408 & -0.883327 \\
\hline 6.8005938 & 1. 2147842 & -0.7790816 \\
\hline 5.0330820 & -2.2403341 & 1.077480 \\
\hline 7.0383524 & -1.0609778 & 0.203715 \\
\hline
\end{tabular}


Table S3. Absolute energy, calculated dipole moment, and coordinates for triazene 3-H-NO $\mathbf{N}_{2}$ (cf. Table 3, Entry 2) at B3LYP/6-31G*-level of theory. ${ }^{8}$

$E(H F)=-1058.8466354$ a.u.

$\mu_{\text {calc }}=10.6$ debye

\begin{tabular}{rll}
\multicolumn{4}{c}{ Atom } \\
- & $--\cdot-\cdot-$ \\
1 & $\mathrm{H}$ & $\mathrm{H} 1$ \\
2 & $\mathrm{C}$ & $\mathrm{C} 1$ \\
3 & $\mathrm{C}$ & $\mathrm{C} 4$ \\
4 & $\mathrm{C}$ & $\mathrm{C} 2$ \\
5 & $\mathrm{C}$ & $\mathrm{C} 6$ \\
6 & $\mathrm{C}$ & $\mathrm{C} 5$ \\
7 & $\mathrm{C}$ & $\mathrm{C} 3$ \\
8 & $\mathrm{H}$ & $\mathrm{H} 6$ \\
9 & $\mathrm{H}$ & $\mathrm{H} 5$ \\
10 & $\mathrm{H}$ & $\mathrm{H} 4$ \\
11 & $\mathrm{~N}$ & $\mathrm{~N} 1$ \\
12 & $\mathrm{~N}$ & $\mathrm{~N} 2$ \\
13 & $\mathrm{C}$ & $\mathrm{C} 7$ \\
14 & $\mathrm{C}$ & $\mathrm{C} 8$ \\
15 & $\mathrm{H}$ & $\mathrm{H} 7$ \\
16 & $\mathrm{H}$ & $\mathrm{H} 9$ \\
17 & $\mathrm{H}$ & $\mathrm{H} 10$ \\
18 & $\mathrm{C}$ & $\mathrm{C} 9$ \\
19 & $\mathrm{H}$ & $\mathrm{H} 8$ \\
20 & $\mathrm{H}$ & $\mathrm{H} 11$ \\
21 & $\mathrm{H}$ & $\mathrm{H} 12$ \\
22 & $\mathrm{~N}$ & $\mathrm{~N} 3$ \\
23 & $\mathrm{~N}$ & $\mathrm{~N} 4$ \\
24 & $\mathrm{~N}$ & $\mathrm{~N} 5$ \\
25 & $\mathrm{C}$ & $\mathrm{C} 10$ \\
26 & $\mathrm{C}$ & $\mathrm{C} 11$ \\
27 & $\mathrm{C}$ & $\mathrm{C} 12$ \\
28 & $\mathrm{C}$ & $\mathrm{C} 13$ \\
29 & $\mathrm{C}$ & $\mathrm{C} 14$ \\
30 & $\mathrm{C}$ & $\mathrm{C} 15$ \\
31 & $\mathrm{H}$ & $\mathrm{H} 3$ \\
32 & $\mathrm{H}$ & $\mathrm{H} 13$ \\
33 & $\mathrm{H}$ & $\mathrm{H} 14$ \\
34 & $\mathrm{H}$ & $\mathrm{H} 15$ \\
35 & $\mathrm{~N}$ & $\mathrm{~N} 6$ \\
36 & $\mathrm{O}$ & 01 \\
37 & $\mathrm{O}$ & $\mathrm{O} 2$ \\
& &
\end{tabular}

\begin{tabular}{|c|c|c|}
\hline$\underset{X}{\operatorname{artesian}}$ & $\underset{Y}{\operatorname{coordinates}}$ & $\begin{array}{c}\text { (Angstroms) } \\
Z\end{array}$ \\
\hline 3.3576505 & -2.9681504 & ๑. 3566272 \\
\hline 3.8194678 & -1.9961930 & $\odot .2180702$ \\
\hline 5.0501493 & 0.5478461 & $-\odot .1769766$ \\
\hline 3.0628207 & -0.8320947 & $\odot .1400132$ \\
\hline 5.2079987 & -1.8704973 & $\odot .0992529$ \\
\hline 5.8117092 & -0.6223068 & $-\odot .0947716$ \\
\hline 3.6700845 & ๑.4199226 & -0.0571073 \\
\hline 5.8258159 & -2.7615511 & $\odot .1544141$ \\
\hline 6.8917587 & -0.5584512 & $-\odot .1864959$ \\
\hline 5.5196357 & 1.5141417 & $-\odot .3303039$ \\
\hline 1.6852464 & -0.6154401 & 0.2258488 \\
\hline 2.6502934 & 1.3588060 & -0.0984335 \\
\hline 1.4309680 & 0.7327189 & ๑. 0584990 \\
\hline 0.7244098 & -1.6748971 & $\odot .5096735$ \\
\hline-0.1304543 & -1.2624601 & 1.0398844 \\
\hline 1.2185701 & -2.4275596 & 1.1301309 \\
\hline ๑.3689193 & -2.1436795 & -0.4139135 \\
\hline 2.8157866 & 2.7869949 & $-\odot .3099235$ \\
\hline 3.4521162 & 3.2111585 & $\odot .4739553$ \\
\hline 1.8278892 & 3.2450037 & $-\odot .2701510$ \\
\hline 3.2725744 & 2.9752596 & -1.2876380 \\
\hline$\odot .3268278$ & 1.4588110 & 0.0545665 \\
\hline$-\odot .8189302$ & $\odot .7500744$ & ๑.0102758 \\
\hline-1.8348544 & 1.5072856 & $\odot .1409192$ \\
\hline-3.0582624 & $\odot .8213245$ & $\odot .0244505$ \\
\hline-5.5919938 & -0.3202520 & -0.1344839 \\
\hline-3.2268220 & $-\odot .4664593$ & $-\odot .5266534$ \\
\hline-4.1912632 & 1.5262426 & 0.4700790 \\
\hline-5.4568012 & ๑. 9612615 & ๑. 4017416 \\
\hline-4.4879218 & -1.0372947 & -0.6038026 \\
\hline-2.3604568 & $-\odot .9992376$ & $-\odot .9003602$ \\
\hline-4.0453015 & 2.5224813 & $\odot .8746031$ \\
\hline-6.3353941 & 1.4901031 & $\odot .7498849$ \\
\hline-4.6390668 & -2.0235124 & -1.0261124 \\
\hline-6.9217006 & $-\odot .9241048$ & $-\odot .2172362$ \\
\hline-7.8789720 & $-\odot .2701569$ & 0.2033139 \\
\hline-7.0124970 & -2.0551378 & -0.7018405 \\
\hline
\end{tabular}


Table S4. Absolute energy, calculated dipole moment, and coordinates for triazene 3-OCCH$-\mathbf{H}$ (cf.

Table 3, Entry 3) at B3LYP/6-31G*-level of theory. ${ }^{8}$

$E(H F)=-1083.3757044$ a.u.

$\mu_{\text {calc }}=5.39$ debye

\begin{tabular}{rll}
\multicolumn{3}{c}{ Atom } \\
- & $-\cdot-\cdot-$ \\
1 & $\mathrm{H}$ & $\mathrm{H} 1$ \\
2 & $\mathrm{C}$ & $\mathrm{C} 1$ \\
3 & $\mathrm{C}$ & $\mathrm{C} 4$ \\
4 & $\mathrm{C}$ & $\mathrm{C} 2$ \\
5 & $\mathrm{C}$ & $\mathrm{C} 6$ \\
6 & $\mathrm{C}$ & $\mathrm{C} 5$ \\
7 & $\mathrm{C}$ & $\mathrm{C} 3$ \\
8 & $\mathrm{H}$ & $\mathrm{H} 4$ \\
9 & $\mathrm{~N}$ & $\mathrm{~N} 1$ \\
10 & $\mathrm{~N}$ & $\mathrm{~N} 2$ \\
11 & $\mathrm{C}$ & $\mathrm{C} 7$ \\
12 & $\mathrm{C}$ & $\mathrm{C} 8$ \\
13 & $\mathrm{H}$ & $\mathrm{H} 7$ \\
14 & $\mathrm{H}$ & $\mathrm{H} 9$ \\
15 & $\mathrm{H}$ & $\mathrm{H} 10$ \\
16 & $\mathrm{C}$ & $\mathrm{C} 9$ \\
17 & $\mathrm{H}$ & $\mathrm{H} 8$ \\
18 & $\mathrm{H}$ & $\mathrm{H} 11$ \\
19 & $\mathrm{H}$ & $\mathrm{H} 12$ \\
20 & $\mathrm{~N}$ & $\mathrm{~N} 3$ \\
21 & $\mathrm{~N}$ & $\mathrm{~N} 4$ \\
22 & $\mathrm{~N}$ & $\mathrm{~N} 5$ \\
23 & $\mathrm{C}$ & $\mathrm{C} 10$ \\
24 & $\mathrm{C}$ & $\mathrm{C} 11$ \\
25 & $\mathrm{C}$ & $\mathrm{C} 12$ \\
26 & $\mathrm{C}$ & $\mathrm{C} 13$ \\
27 & $\mathrm{C}$ & $\mathrm{C} 14$ \\
28 & $\mathrm{C}$ & $\mathrm{C} 15$ \\
29 & $\mathrm{H}$ & $\mathrm{H} 3$ \\
30 & $\mathrm{H}$ & $\mathrm{H} 13$ \\
31 & $\mathrm{H}$ & $\mathrm{H} 14$ \\
32 & $\mathrm{H}$ & $\mathrm{H} 15$ \\
33 & $\mathrm{O}$ & 01 \\
34 & $\mathrm{O}$ & 02 \\
35 & $\mathrm{C}$ & $\mathrm{C} 16$ \\
36 & $\mathrm{H}$ & $\mathrm{H} 5$ \\
& &
\end{tabular}

$\underset{\mathrm{X}}{\mathrm{Cartesian}} \underset{\mathrm{Y}}{\mathrm{C}} \mathrm{Z}_{\mathrm{Z}}$

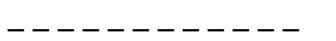

$-2.1465577$

$-2.1946880$

$-1.1609012$

1.5647907

$-0.1427751$

$-\odot .7999637$

0.5590017

1.1968204

2. 5923431

$-0.1903019$

1.9366034

1. 0965888

$-1.4316768$

$-1.1989855$

$-1.9664853$

$-2.0668189$

3. 3753956

3. 8835129

3.6447574

3.6772110

1. 6187761

0.7290054

1. 3140578

0.4391941

$-1.1124791$

$-0.8325893$

0.9299219

0.1563776

$-1.5978270$

$-1.1967709$

1. 9215929

0.5466016

$-2.5768820$

$-1.6950299$

0.9123942

$-3.0750427$

$-3.3112569$
$-0.0566186$

๑. 0592547

๑. 3856499

0.1120741

$\odot .1773617$

$\odot .3332742$

0.2788491

0.5186357

0.0204193

0.2920635

$\odot .1379039$

$-0.2135050$

$-0.4409156$

$-1.0494209$

0.6798204

0.4549671

$-0.3255320$

0.3697803

1.4373254

$\odot .1040505$

$\odot .0829285$

$-0.0962637$

$-0.0598916$

$-0.0788944$

0.5413382

$-0.6437258$

$-0.6622506$

0.5269559

1. 0286633

$-1.0855445$

$-1.1271699$

1. 0003959

$\odot .1632236$

$\odot .5077418$

0.0659987

$-\odot .8781274$ 


$\begin{array}{lll}37 & \mathrm{H} & \mathrm{H} 17 \\ 38 & \mathrm{H} & \mathrm{H} 18 \\ 39 & \mathrm{C} & \mathrm{C} 17 \\ 40 & \mathrm{H} & \mathrm{H} 2 \\ 41 & \mathrm{H} & \mathrm{H} 19 \\ 42 & \mathrm{H} & \mathrm{H} 20 \\ 43 & \mathrm{H} & \mathrm{H} 21\end{array}$

$-5.4753605$

$-3.8942046$

$-6.3077205$

$-5.9197960$

$-7.2952874$

$-6.3850041$

8. 1026367
$-3.5991416$

- 3.4073013

$\odot .8054621$

1. 4346240

1.1666705

$-0.2309504$

$-1.7138355$ $\odot .0986394$

$\odot .9062777$

$-0.6555766$

$-1.4683406$

$-0.3590284$

$-0.9990283$

$-0.0837591$ 
Table S5. Absolute energy, calculated dipole moment, and coordinates for triazene $\mathbf{3}-\mathbf{O C H}_{3}-\mathbf{N O}_{2}$ (cf.

Table 3, Entry 4) at B3LYP/6-31G*-level of theory. ${ }^{8}$

$E(H F)=-1287.8812702$ a.u.

$\mu_{\text {calc }}=12.2$ debye

\begin{tabular}{rll}
\multicolumn{3}{c}{ Atom } \\
-1 & $\mathrm{H}$ & $\mathrm{H} 1$ \\
2 & $\mathrm{C}$ & $\mathrm{C} 1$ \\
3 & $\mathrm{C}$ & $\mathrm{C} 4$ \\
4 & $\mathrm{C}$ & $\mathrm{C} 2$ \\
5 & $\mathrm{C}$ & $\mathrm{C} 6$ \\
6 & $\mathrm{C}$ & $\mathrm{C} 5$ \\
7 & $\mathrm{C}$ & $\mathrm{C} 3$ \\
8 & $\mathrm{H}$ & $\mathrm{H} 4$ \\
9 & $\mathrm{~N}$ & $\mathrm{~N} 1$ \\
10 & $\mathrm{~N}$ & $\mathrm{~N} 2$ \\
11 & $\mathrm{C}$ & $\mathrm{C} 7$ \\
12 & $\mathrm{C}$ & $\mathrm{C} 8$ \\
13 & $\mathrm{H}$ & $\mathrm{H} 7$ \\
14 & $\mathrm{H}$ & $\mathrm{H} 9$ \\
15 & $\mathrm{H}$ & $\mathrm{H} 10$ \\
16 & $\mathrm{C}$ & $\mathrm{C} 9$ \\
17 & $\mathrm{H}$ & $\mathrm{H} 8$ \\
18 & $\mathrm{H}$ & $\mathrm{H} 11$ \\
19 & $\mathrm{H}$ & $\mathrm{H} 12$ \\
20 & $\mathrm{~N}$ & $\mathrm{~N} 3$ \\
21 & $\mathrm{~N}$ & $\mathrm{~N} 4$ \\
22 & $\mathrm{~N}$ & $\mathrm{~N} 5$ \\
23 & $\mathrm{C}$ & $\mathrm{C} 10$ \\
24 & $\mathrm{C}$ & $\mathrm{C} 11$ \\
25 & $\mathrm{C}$ & $\mathrm{C} 12$ \\
26 & $\mathrm{C}$ & $\mathrm{C} 13$ \\
27 & $\mathrm{C}$ & $\mathrm{C} 14$ \\
28 & $\mathrm{C}$ & $\mathrm{C} 15$ \\
29 & $\mathrm{H}$ & $\mathrm{H} 3$ \\
30 & $\mathrm{H}$ & $\mathrm{H} 13$ \\
31 & $\mathrm{H}$ & $\mathrm{H} 14$ \\
32 & $\mathrm{H}$ & $\mathrm{H} 15$ \\
33 & $\mathrm{O}$ & 01 \\
34 & $\mathrm{O}$ & 02 \\
35 & $\mathrm{C}$ & $\mathrm{C} 16$ \\
36 & $\mathrm{H}$ & $\mathrm{H} 5$ \\
37 & $\mathrm{H}$ & $\mathrm{H} 17$
\end{tabular}

Cartesian Coordinates (Angstroms)

\begin{tabular}{|c|c|c|}
\hline & & \\
\hline X & Y & Z \\
\hline-------- & ---------- & ----------- \\
\hline-2.3732828 & -2.2561783 & $-\odot .2404470$ \\
\hline-2.7468512 & -1.2576665 & -0.0537524 \\
\hline-3.6997837 & 1.3743054 & ๑. 4781917 \\
\hline-1.8774353 & -0.1728978 & 0.0475372 \\
\hline-4.1149261 & -1.0113250 & ๑. 1185581 \\
\hline-4.5864689 & 0.3033934 & 0.3758962 \\
\hline-2.3409556 & 1.1181698 & 0.3175217 \\
\hline - 4.0923980 & 2.3624502 & $\odot .6904770$ \\
\hline$-\odot .4879950$ & - ๑. . 0993453 & -0.0763258 \\
\hline-1.2276392 & 1.9463152 & ๑. 3655086 \\
\hline$-\odot .0876021$ & 1.2071755 & $\odot .1364731$ \\
\hline 0.3400396 & -1.2460719 & $-\odot .4282964$ \\
\hline 1.2786287 & $-\odot .8991728$ & $-\odot .8513934$ \\
\hline-0.2014114 & -1.8537807 & -1.1599374 \\
\hline 0.5589741 & -1.8556631 & 0.4557998 \\
\hline-1.2373494 & 3.3721033 & 0.6458085 \\
\hline-1.8593058 & 3.8944203 & $-\odot .0889806$ \\
\hline$-\odot .2095957$ & 3.7275105 & ๑. 5799297 \\
\hline-1.6322460 & 3.5583655 & 1.6504862 \\
\hline 1.0859817 & 1.8206389 & ๑. 1279319 \\
\hline 2.1574400 & 1.0094426 & 0.0881346 \\
\hline 3.2378287 & 1.6749798 & -0.0440801 \\
\hline 4.3910262 & 0.8713498 & -0.0156682 \\
\hline 6.8047153 & - ०. 5171764 & - ๑. .032540๑ \\
\hline 4.4571414 & -0.4512565 & $\odot .4724960$ \\
\hline 5.5680027 & 1.4836175 & $-\odot .4856825$ \\
\hline 6.7728042 & ○. 7962839 & -0.5047839 \\
\hline 5.6574430 & -1.1446522 & 0.4612787 \\
\hline 3.5607149 & -0.9137900 & ๑. 8683571 \\
\hline 5.5028593 & 2.5071972 & $-\odot .8399699$ \\
\hline 7.6829739 & 1.2534695 & $-\odot .8726820$ \\
\hline 5.7294714 & -2.1596100 & ๑. 8333397 \\
\hline-5.0774944 & -1.9771945 & $\odot .0720376$ \\
\hline-5.9185220 & ๑. 5383259 & ๑. 6050899 \\
\hline-4.6715191 & -3.3253669 & -0.1052727 \\
\hline-4.1718464 & -3.4749436 & -1.0721497 \\
\hline-5.5876862 & -3.9175689 & -0.0779890 \\
\hline
\end{tabular}




$\begin{array}{lll}38 & \mathrm{H} & \mathrm{H} 18 \\ 39 & \mathrm{C} & \mathrm{C} 17 \\ 40 & \mathrm{H} & \mathrm{H} 2 \\ 41 & \mathrm{H} & \mathrm{H} 19 \\ 42 & \mathrm{H} & \mathrm{H} 20 \\ 43 & \mathrm{~N} & \mathrm{~N} 6 \\ 44 & 0 & 03 \\ 45 & 0 & 04\end{array}$

$-4.0036939$

$-3.6523360$

$\odot .7027542$

$-6.7713458$

0.4372344

$-0.5397999$

$-6.4641186$

1.1489944

$-1.3179476$

$-7.7738592$

๑. 6936965

$-0.1902350$

$-6.7742186$

$-0.5775576$

$-0.9493582$

8.0686496

$-1.2514720$

$-0.0448529$

9.0669070

$-0.6750564$

$-0.4839777$

8. 0679489

$-2.4093575$

$\odot .3825148$ 


\section{References:}

1 Gehrhus, B.; Hitchcock, P. B.; Pongtavornpinyo, R.; Zhang, L. Dalton Trans. 2006, 1847.

2 Daniele, S.; Drost, C.; Gehrhus, B.; Hawkins, S. M.; Hitchcock, P. B.; Lappert, M. F.; Merle, P. G.; Bott, S. G. J. Chem. Soc., Dalton Trans. 2001, 21, 3179.

3 Myes, T. L.; Diver, S. T.; Richard, J. P.; Rivas, F. M.; Toth, K. J. Am. Chem. Soc. 2004, 126, 4366.

4 Hadei, N.; Kantchev, E. A. B.; O’Brien, C. J.; Organ, M. G. Org. Lett. 2005, 7, 1991.

5 Starikova, O. V.; Dolgushin, G. V.; Larina, L. I.; Ushakov, P. E.; Komarova, T. N.; Lopyrev, V. A. Russ. J. Org. Chem. 2003, 39, 1467.

6 Gehrhus, B.; Hitchcock, P. B.; Lappert, M. F. J. Chem. Soc., Dalton Trans. 2000, 3094.

7 Anslyn, E. V.; Dougherty, D. A. Modern Physical Organic Chemistry; University Science Books: Sausalito, CA, 2006; pp 382-390.

8 Calculations were performed at the B3LYP/6-31G* level of density functional theory, as implemented in the Spartan 2004 software package (Wavefunction, Irvine, CA 92612). 


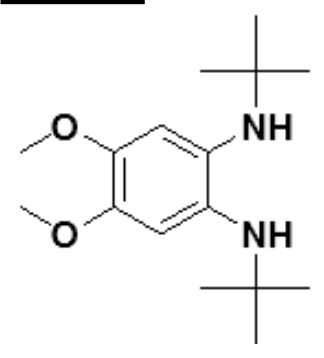

B

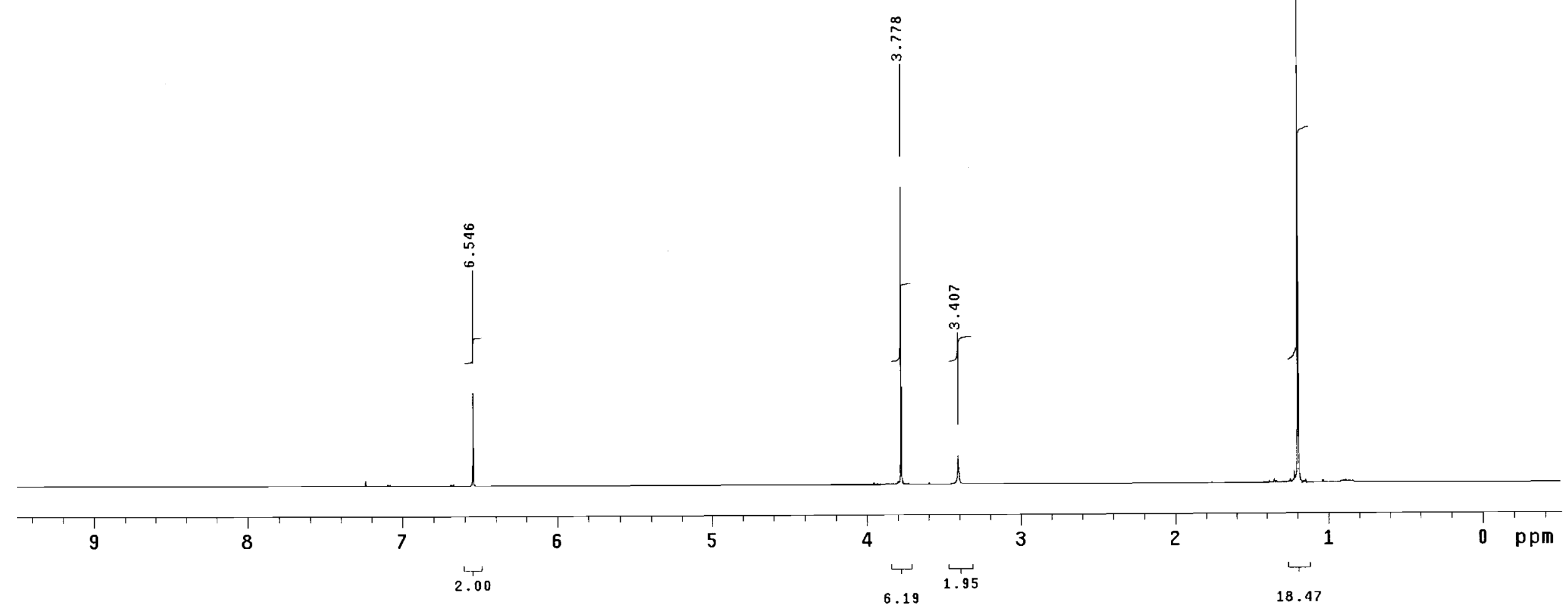

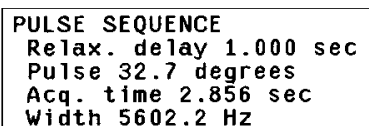

Acq. $t$ ime $2.856 \mathrm{sec}$

width $5602.2 \mathrm{~Hz}$
13 repetitions

OBSERVE

400.2669856

DATA PROCESSING

tine broadening $0.1 \mathrm{~Hz}$

Total time 1 minute 

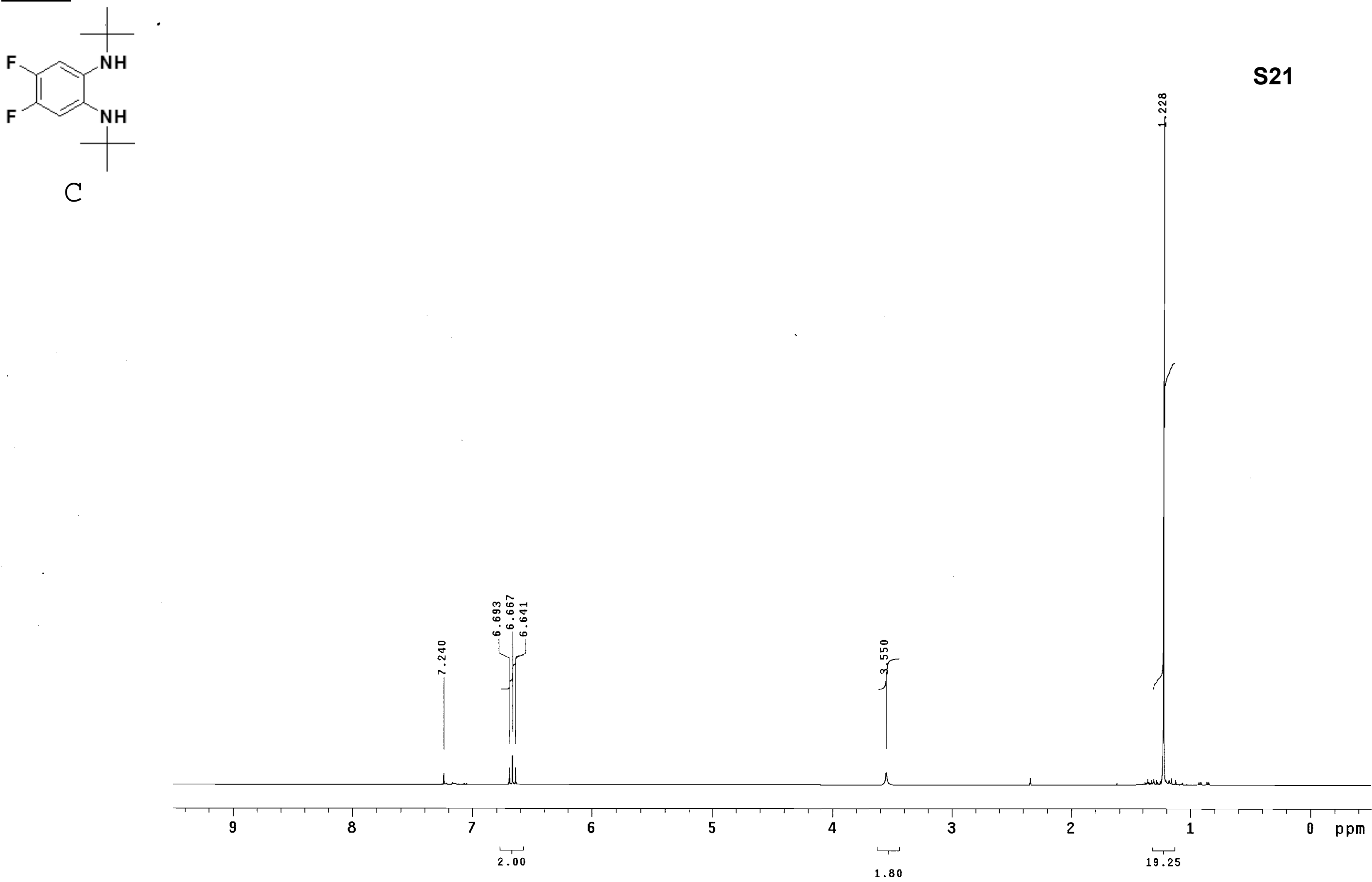

\begin{tabular}{|c|c|c|c|}
\hline $\begin{array}{l}\text { PULSE SEQUENCE } \\
\text { Relax. delay } 1.000 \text { sec } \\
\text { Pulse. } 32.7 \text { degrees } \\
\text { Acq. time } 2.856 \text { sec } \\
\text { Width } 5602.2 \text { Hz } \\
6 \text { repetitions }\end{array}$ & OBSERVE H1, 400.2669859 & $\begin{array}{l}\text { DATA PROCESSING } \\
\text { Line broadening } 0.1 \mathrm{~Hz} \\
\text { FT size } 32768 \\
\text { Total time } 1 \text { minute }\end{array}$ & $\begin{array}{l}\text { dmk092106-3 } \\
1,2-d i(\text { NHtBu) } \\
4,5-d i f \text { ituorobenzene } \\
\text { crude } \\
\text { Pulse Sequence: s2pul } \\
\text { Solvent: CDC13 } \\
\text { Ambient temperature } \\
\end{array}$ \\
\hline
\end{tabular}




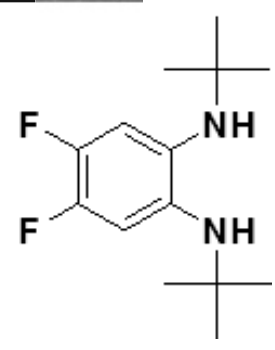

C

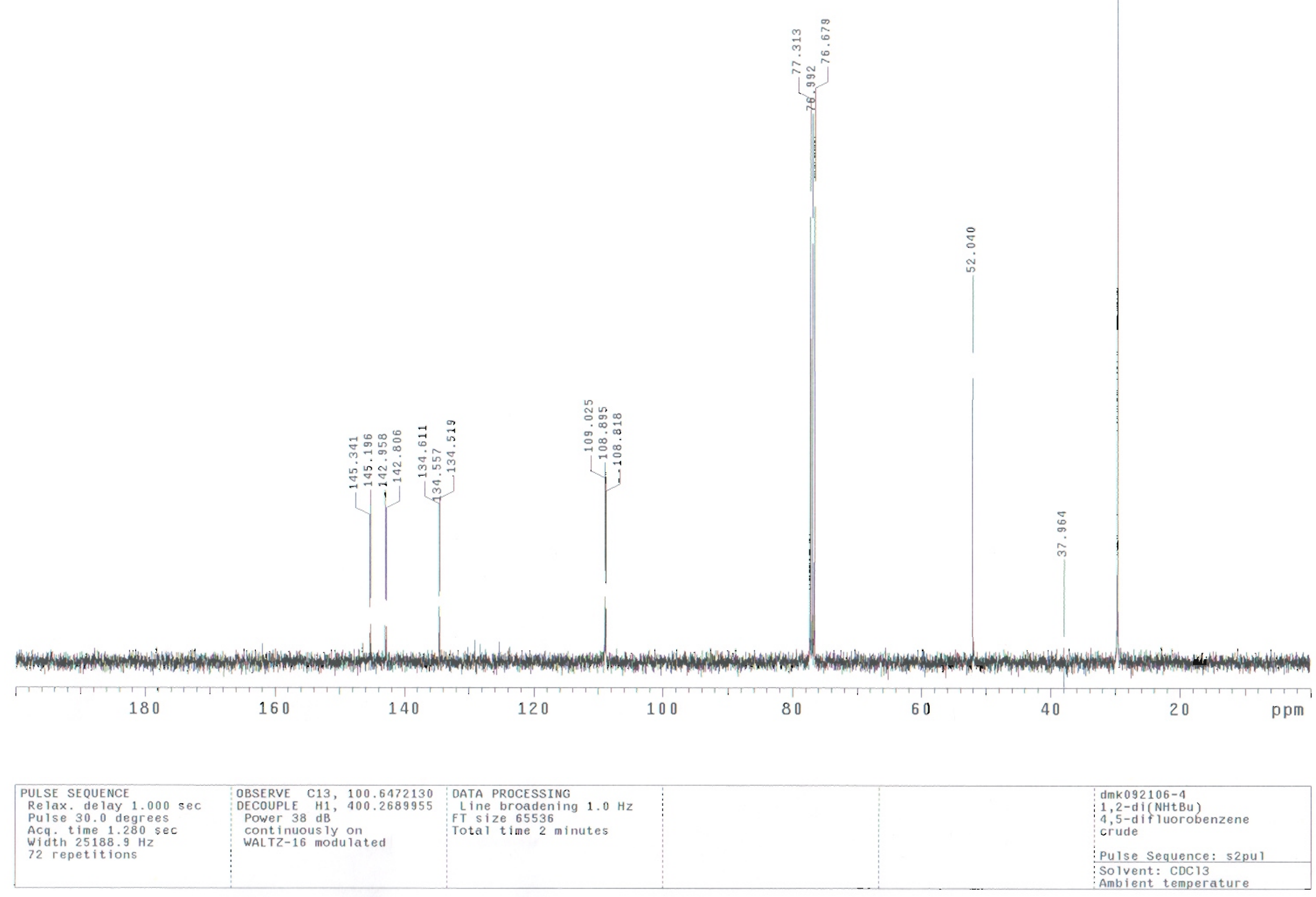



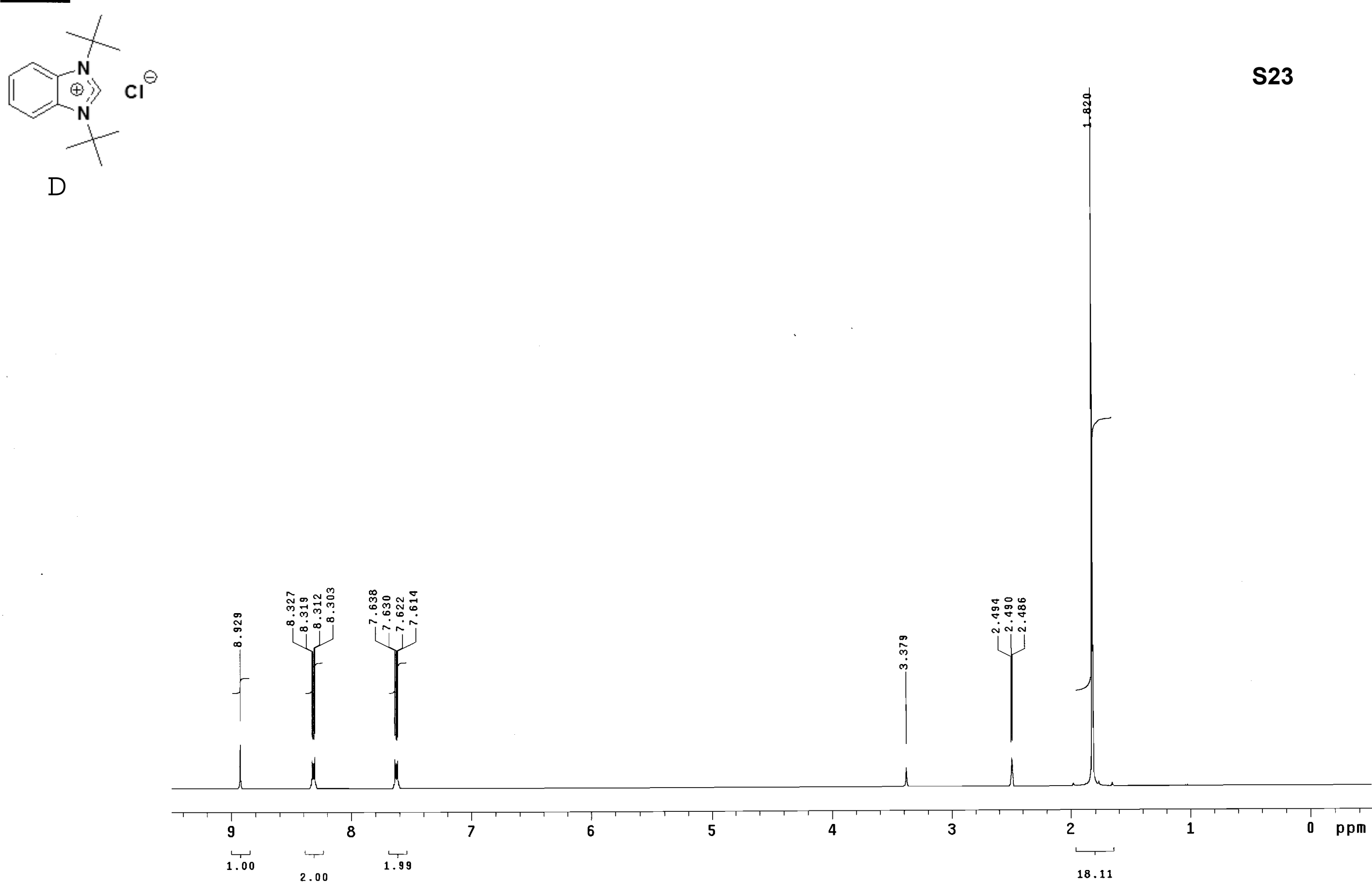

PULSE SEQUENCE

OBSERVE

H1, 400.2688760 DATA PROCESSING

Line broadening $0.1 \mathrm{~Hz}$

Pulse 32.7 degrees

Acq. time $2.856 \mathrm{sec}$

width $5602.2 \mathrm{~Hz}$
repetitions

Total time 1 minute
Tol

dmk091206-5
$\mathrm{N}, \mathrm{N}-\mathrm{tBubenzimdazole} \mathrm{Cl}$

Pulse Sequence: s2pur

Solvent: DMSO

Ambient temperature 


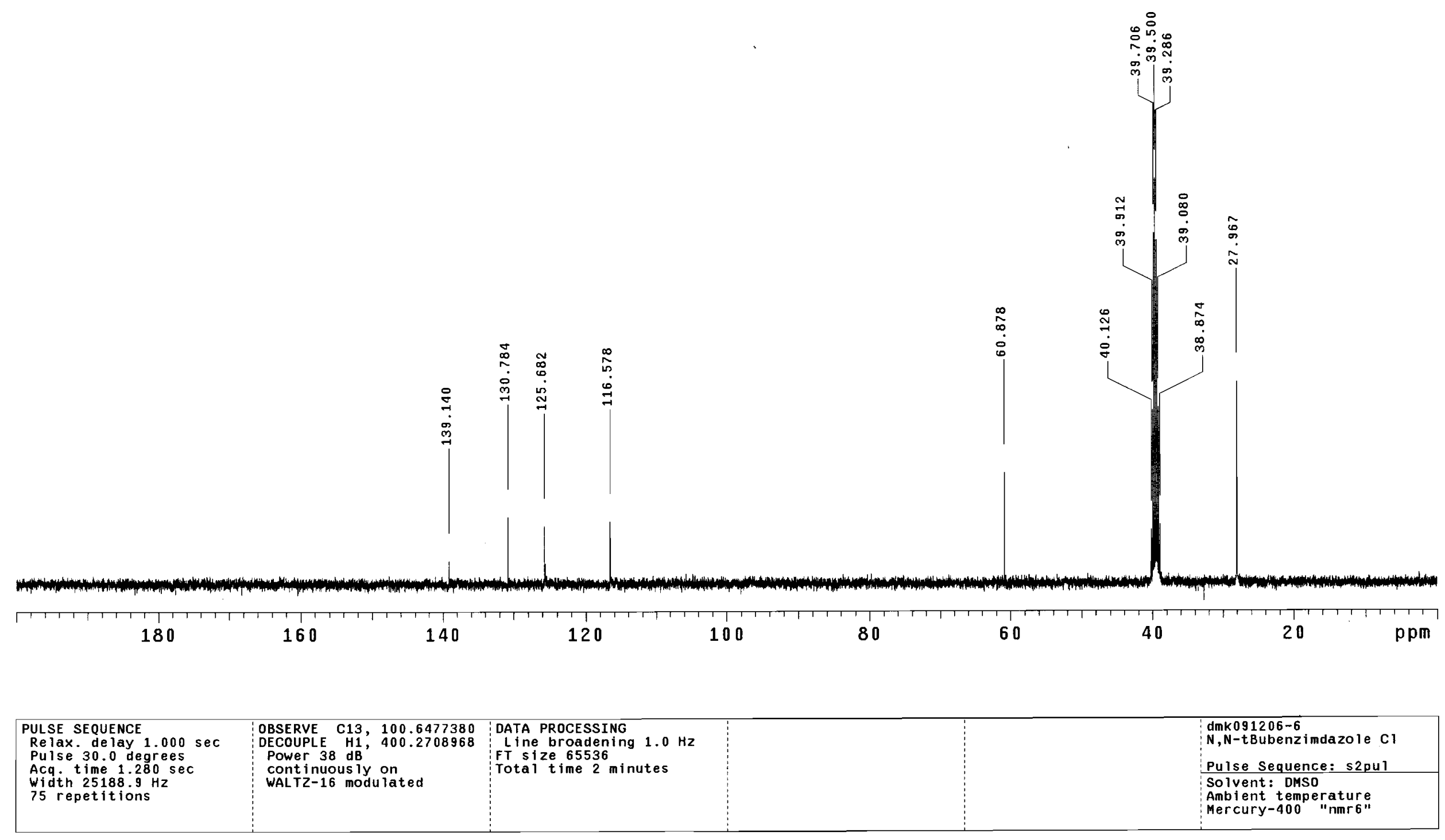




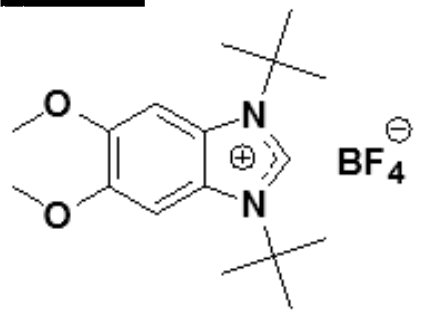

E

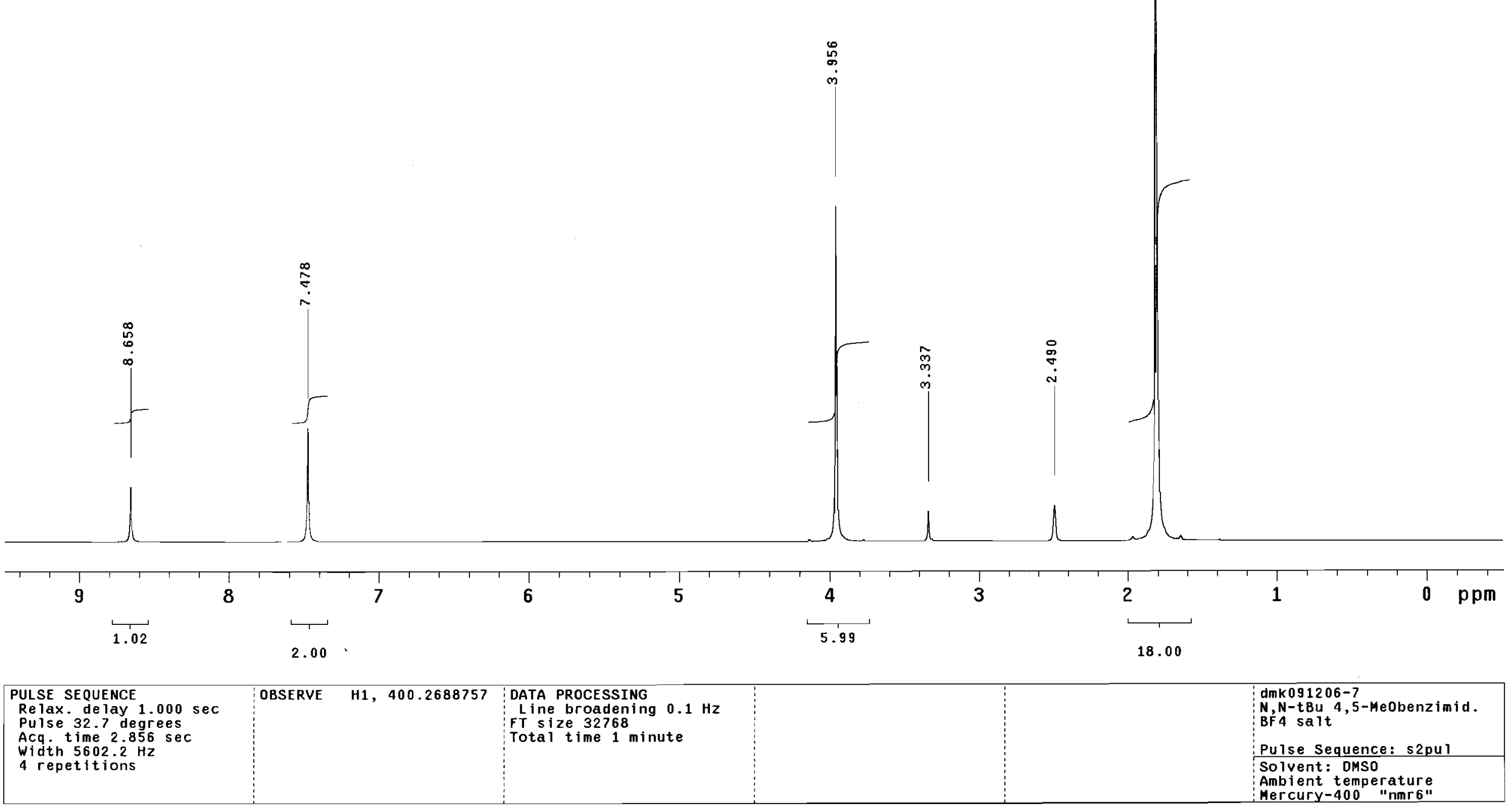


$\overbrace{0}^{N}$

E

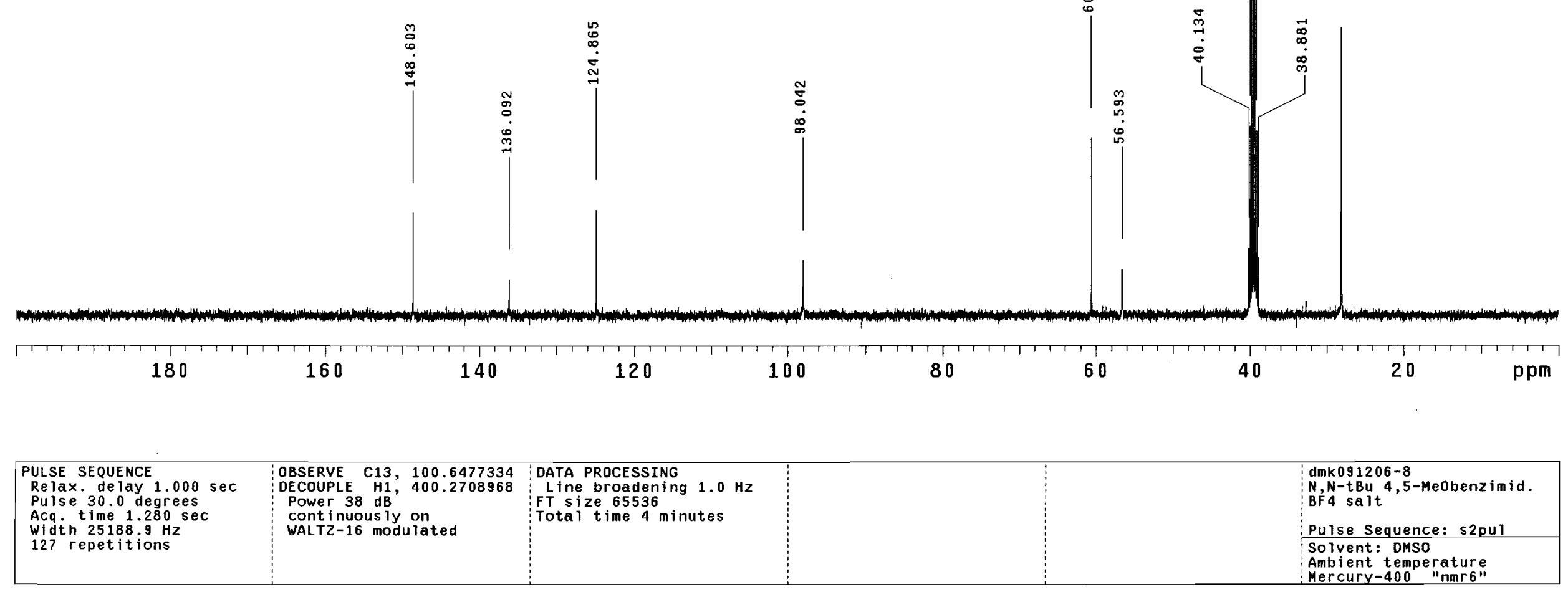



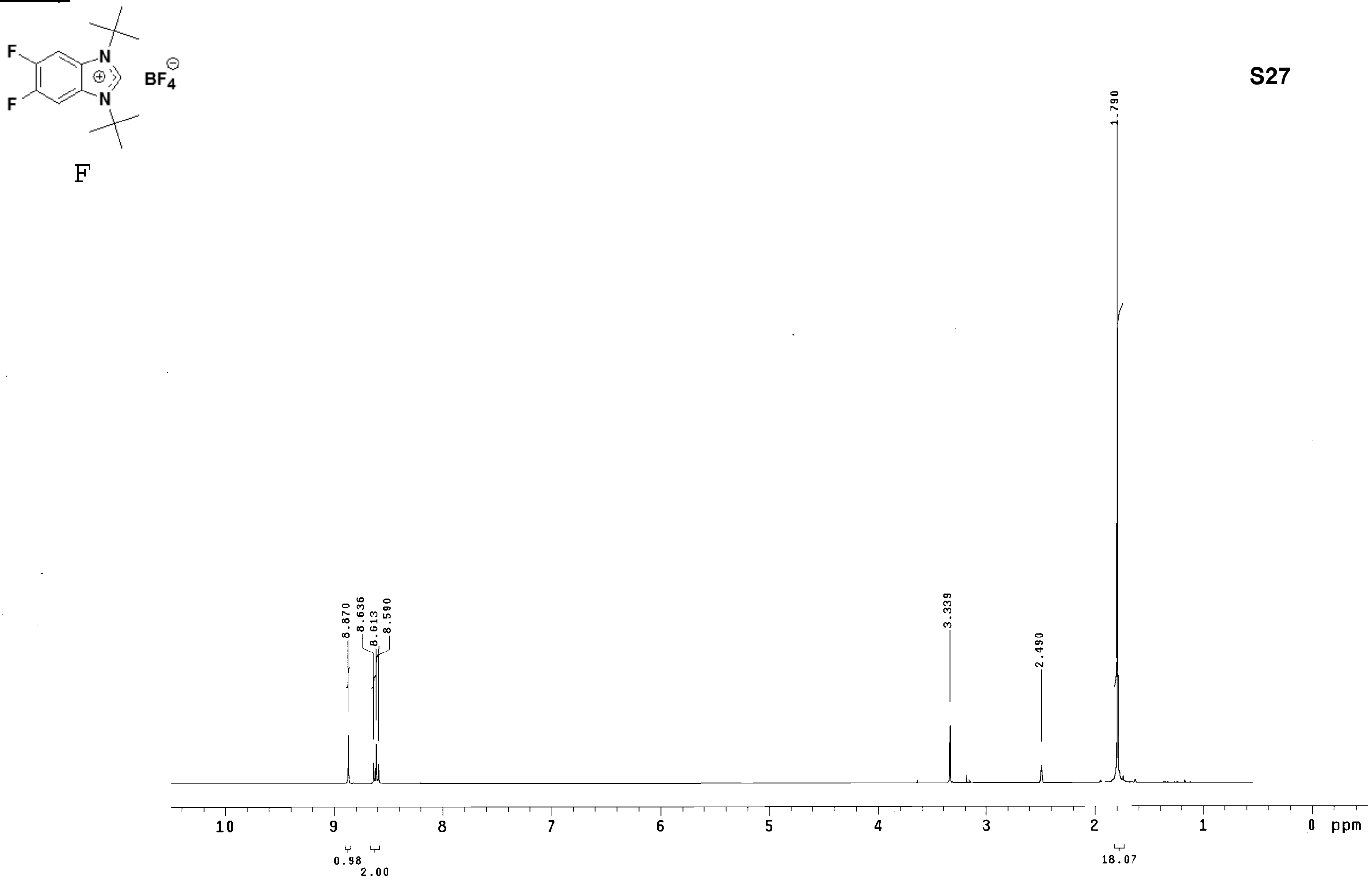

\begin{tabular}{|l|l|l|l} 
PULSE SEQUENCE & OBSERVE H1, 400.2688764 & DATA PROCESSING
\end{tabular}

Relax. delay $1.000 \mathrm{sec}$
Pulse 32.7 degrees

Acq time 2.856 se

Line broadening $0.1 \mathrm{~Hz}$

FT size 32768
Total time 1 minute

dmk092306-1
difluoro

Width 5602.2

dif luor

$R=t B u$

Pulse Sequence: s2pu Solvent: DMSO 

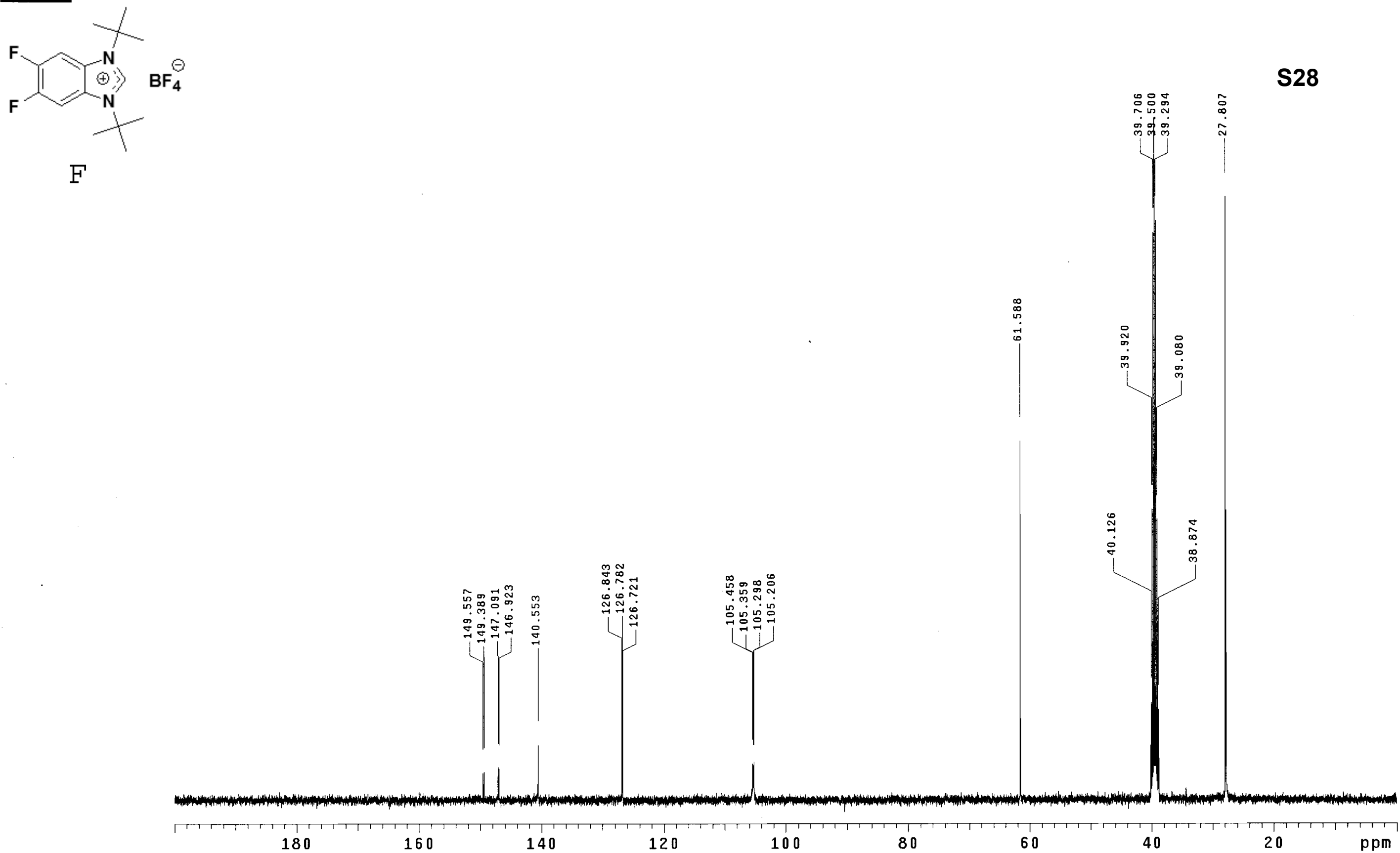

PULSE SEQUENCE

Relax delay $1.000 \mathrm{sec}$

OBSERVE C13, 100.6477326 DATA PROCESSING

400.2708968 Dine broadening $1.0 \mathrm{~Hz}$

Pulse 30.0 degrees

cont inuous iy on
WALT2-16 modulated

Width 25188.9 Hz

Total time 11 minutes

dmk092306
difluoro

benzimidazoli um BF 4

$R=t B u$

Pulse sequence: s2pu Solvent: DMSO
Ambient temperature 


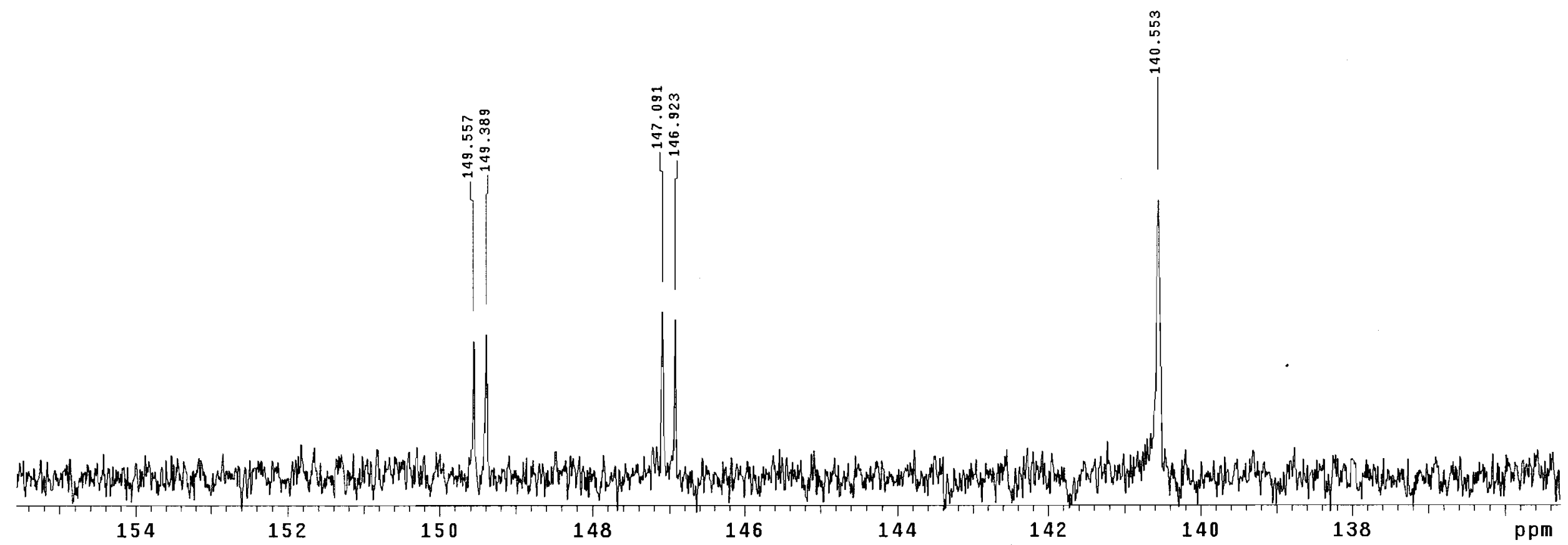

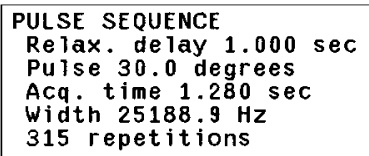

$\begin{array}{ll}\text { OBSERVE } & \text { C13, } \\ \text { DECOUPLE } & 100.6477326 \\ \text { H1 } & 400.2708968\end{array}$

Power $38 \mathrm{~d}$

continuous ly on
WAL $Z-16$ modulated
DATA PROCESSING $1.0 \mathrm{~Hz}$
FT 512 ize 65336
Total time 12 minutes

Width $25188.9 \mathrm{~Hz}$
315 repetitions dmk092306-2

benzimidazolium BF 4

$R=t B u$

Pulse Sequence: s2pu Solvent: DMSO
Ambient temperatur 

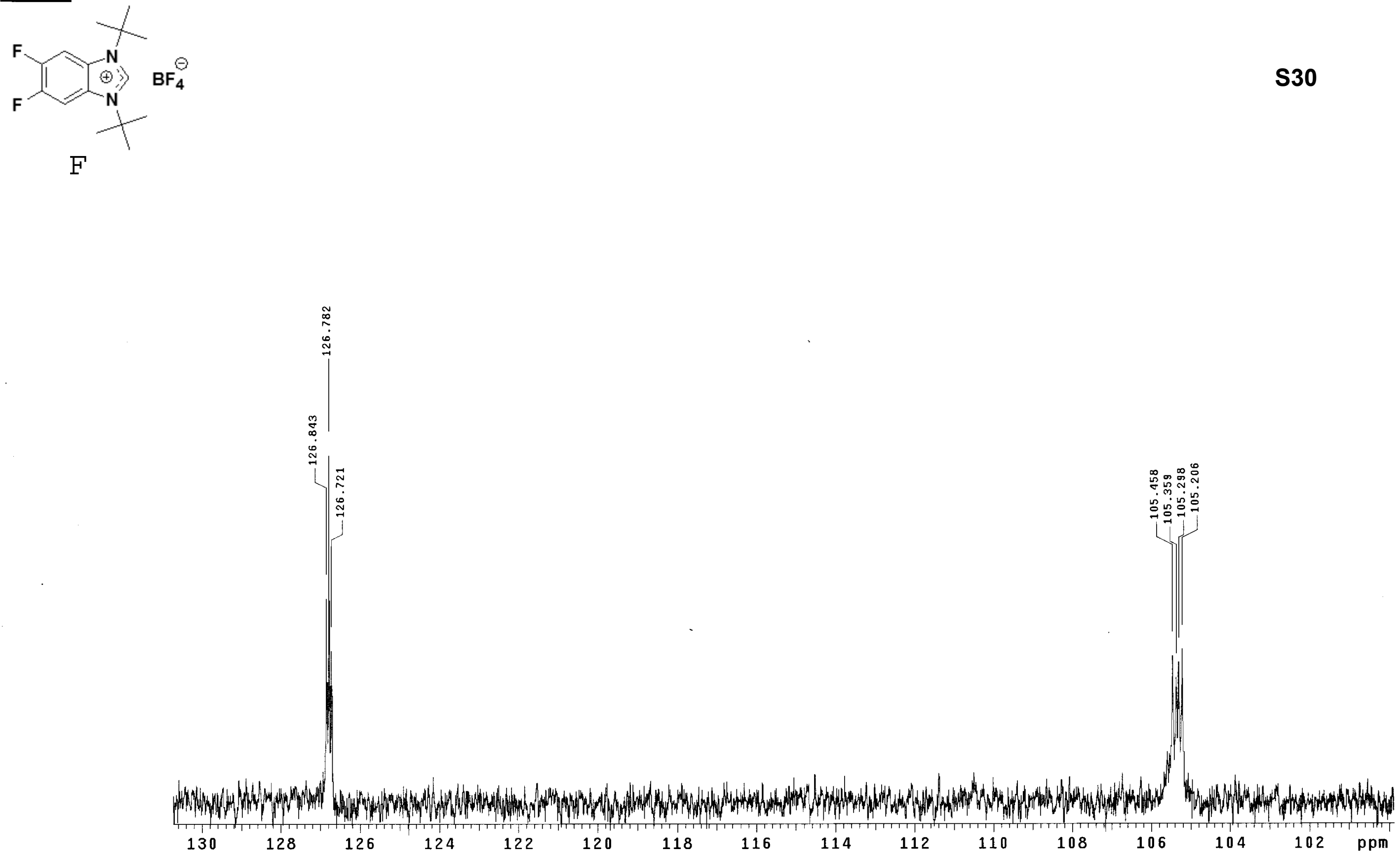

\begin{tabular}{|c|c|c|c|}
\hline $\begin{array}{l}\text { PULSE SEQUENCE } \\
\text { Relax. delay } 1.000 \text { sec } \\
\text { Pulse } 30.0 \text { degrees } \\
\text { Aqq. time } 1.280 \mathrm{sec} \\
\text { Width } 25188.9 \mathrm{~Hz} \\
318 \text { repetitions }\end{array}$ & $\begin{array}{l}\text { OBSERVE C13, 100.6477326 } \\
\text { DECOUPLE H1, } 400.2708968 \\
\text { POWer } 38 \mathrm{~dB} \\
\text { Cont inuous } 1 y \text { on } \\
\text { WALTZ-16 modulated }\end{array}$ & $\begin{array}{l}\text { DATA PROCESSING } \\
\text { Line broadening } 1.0 \mathrm{~Hz} \\
\text { FT size } 65536 \\
\text { Total time } 12 \text { minutes }\end{array}$ & $\begin{array}{l}\text { dmk092306-2 } \\
\text { difluoro } \\
\text { benzimidazolium BF } 4 \\
R=\text { tBu } \\
\text { Pulse Sequence: s2pul } \\
\text { Solvent: DMSO } \\
\text { Ambient temperature }\end{array}$ \\
\hline
\end{tabular}




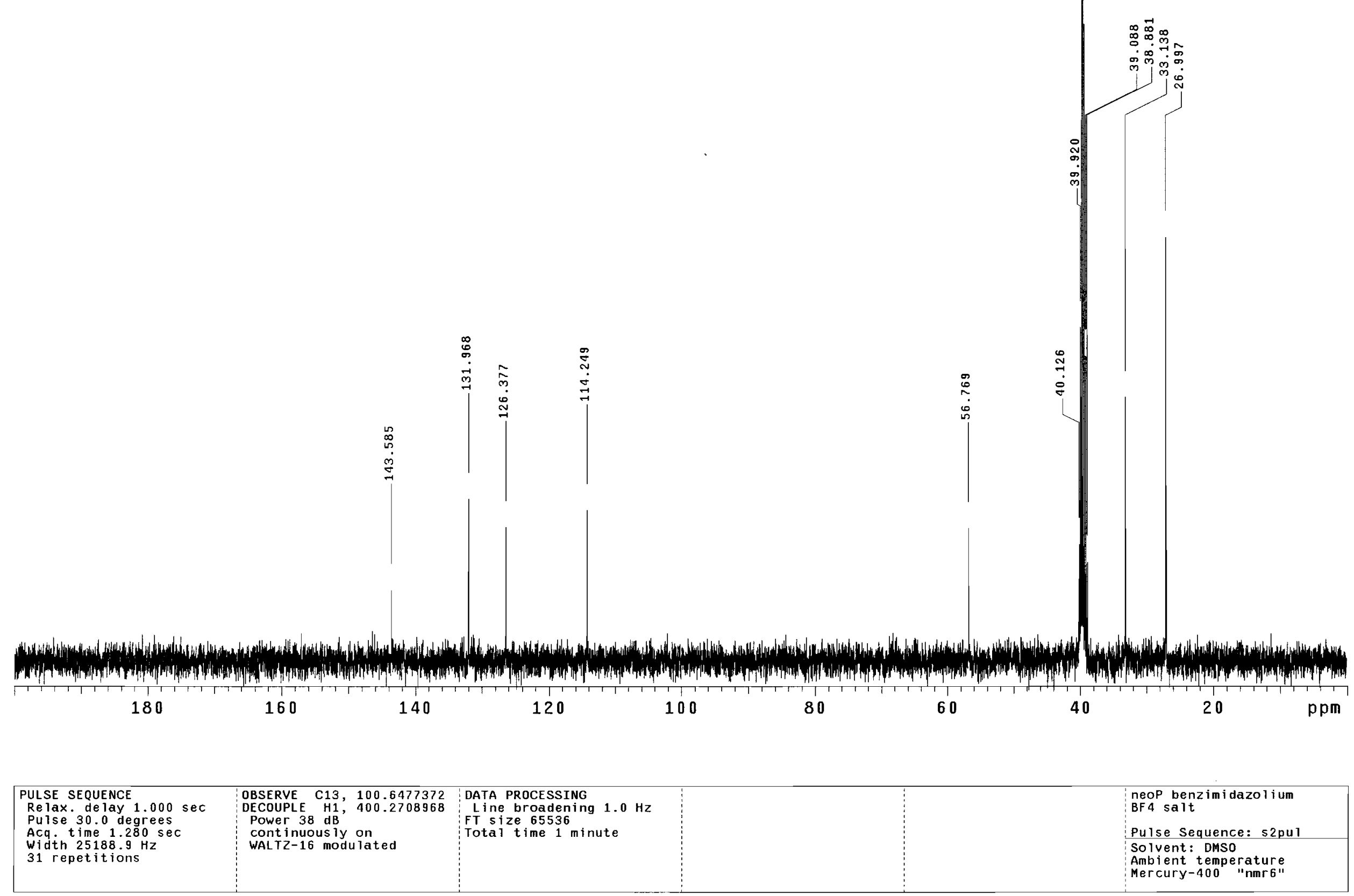



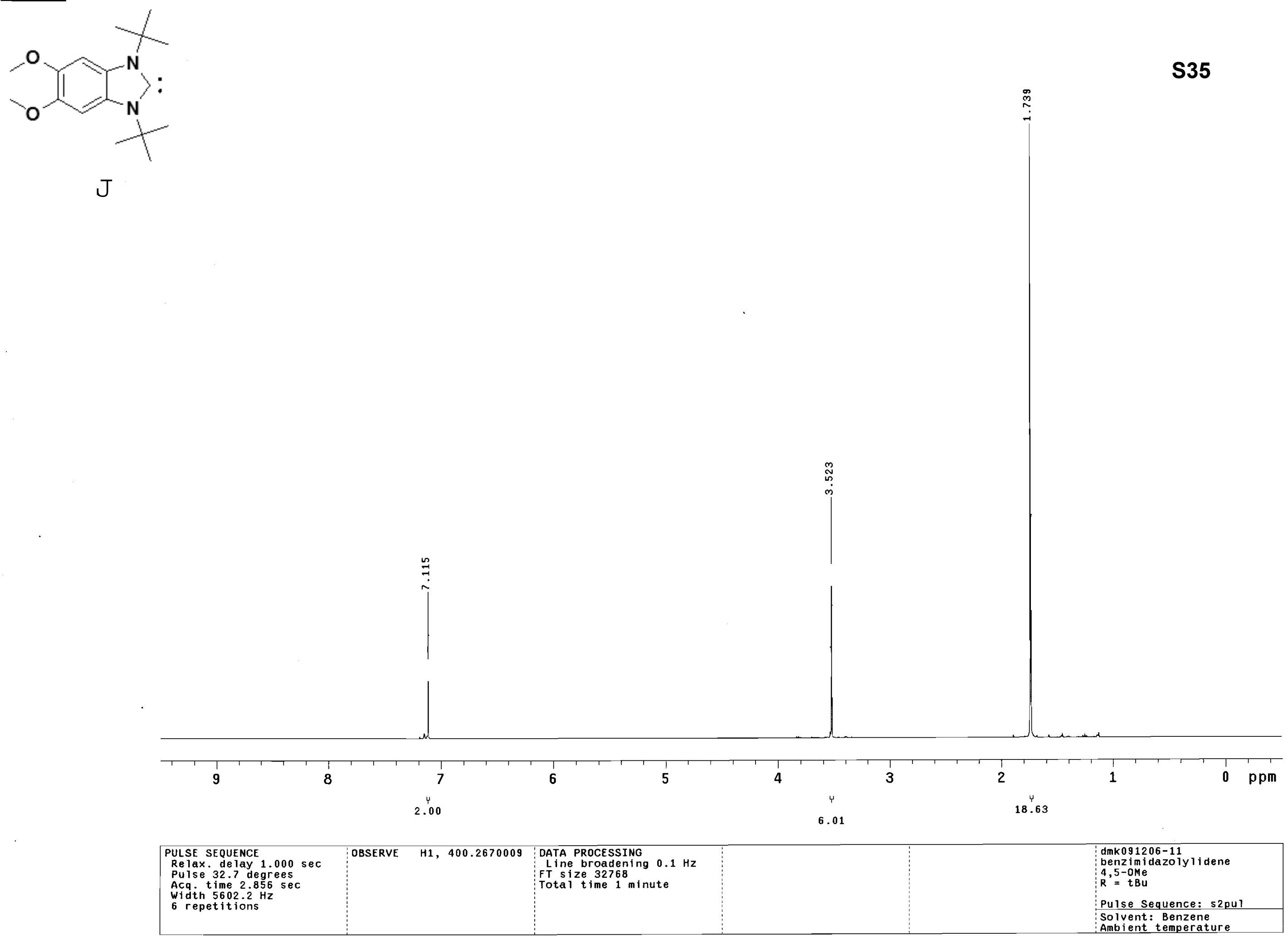
$\overbrace{N}^{\mathrm{N}}$

K

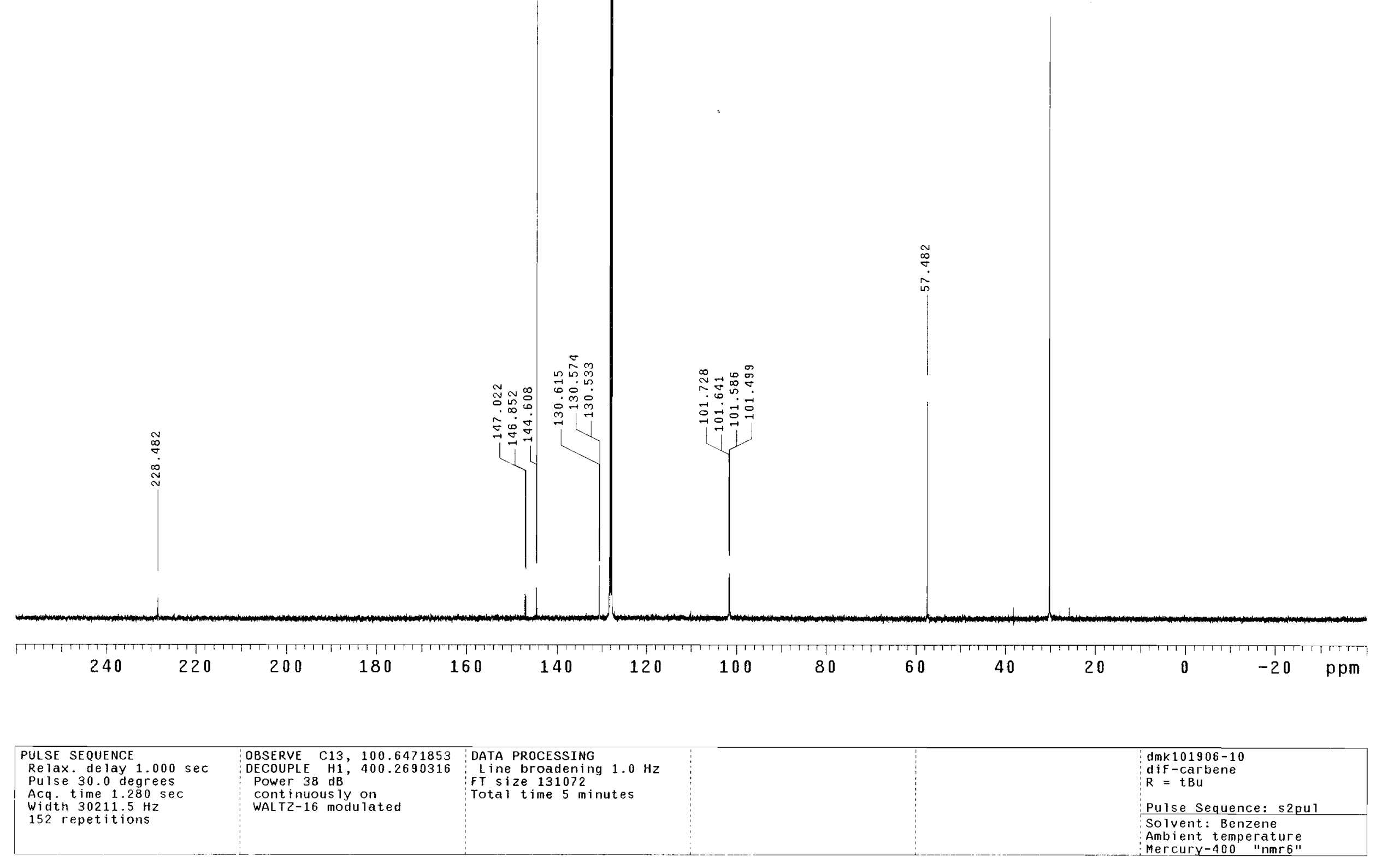




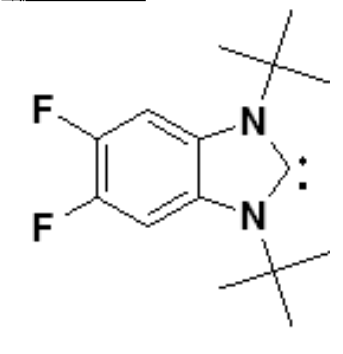

$\mathrm{K}$

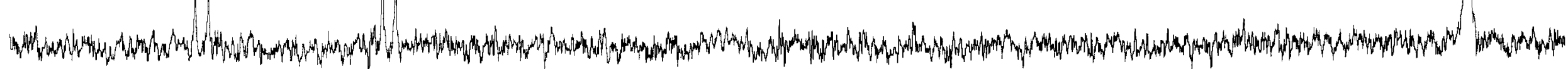

\begin{tabular}{llllllllllllllll}
\hline 148 & 146 & 144 & 142 & 140 & 138 & 136 & 134 & 132 & $\mathrm{ppm}$
\end{tabular}

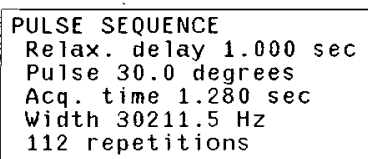

dmk101906-10 dmk $101906-10$
diF-carbene $R=\mathrm{tBu}$

Purse Sequence: s2pu Solvent: Benzene Ambient temperature
Mercury-400 "nmrs" 


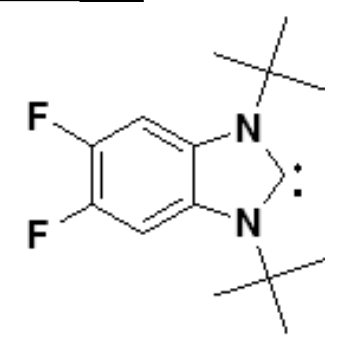

K

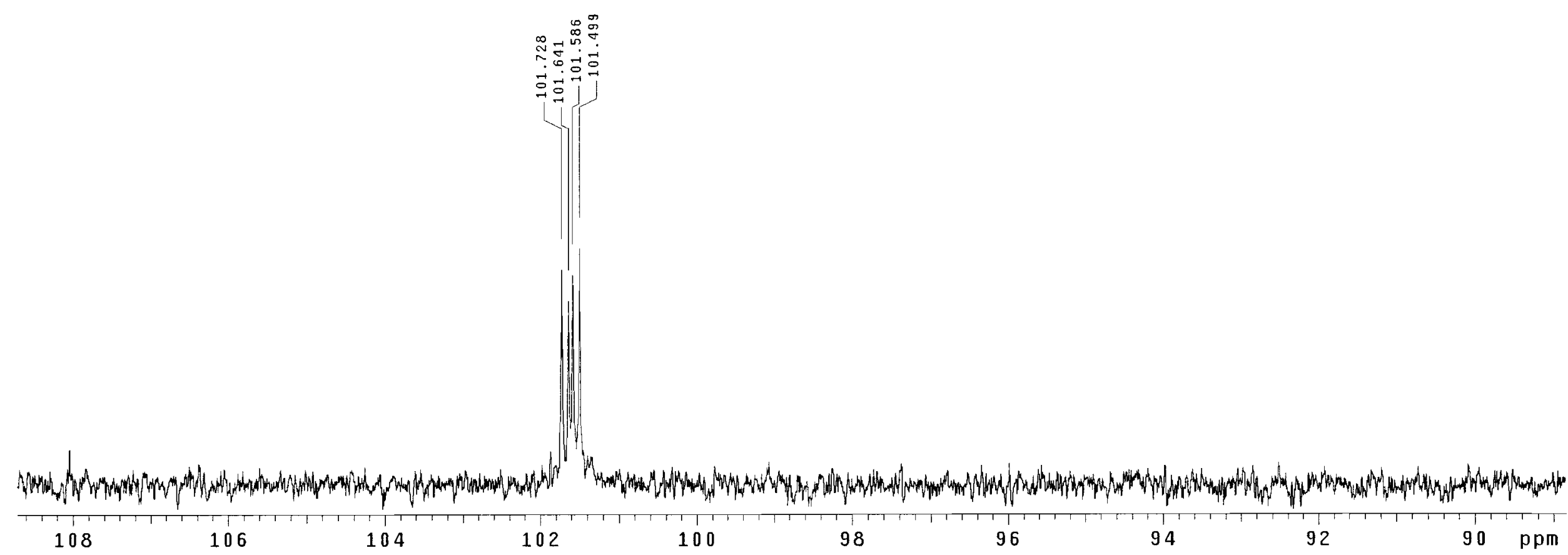

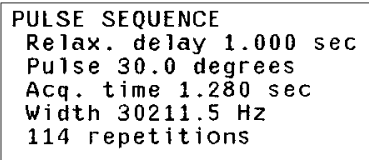

OBSERVE $\mathrm{C} 13, \quad 100.6471853$

Power $38 \mathrm{~d}$

cont inuous $1 y$ on

DATA PROCESSING
Line broadening $1.0 \mathrm{~Hz}$
ST size 131072 m
Total time 4 minutes

dmk101906-10 dif-carben
$R=t B u$

Pulse Sequence: s2pu Solvent: Benzene Ambient temperatur
Mercury-400 "nmra" 


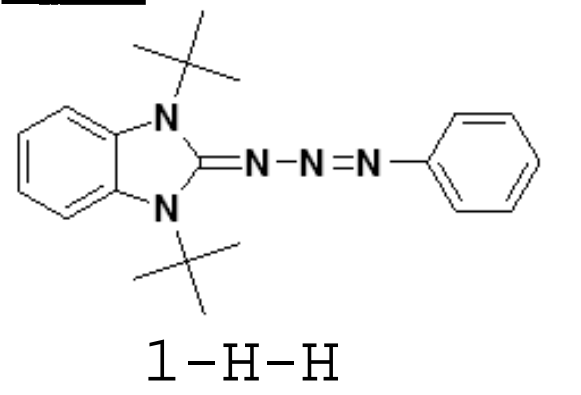

$1-\mathrm{H}-\mathrm{H}$

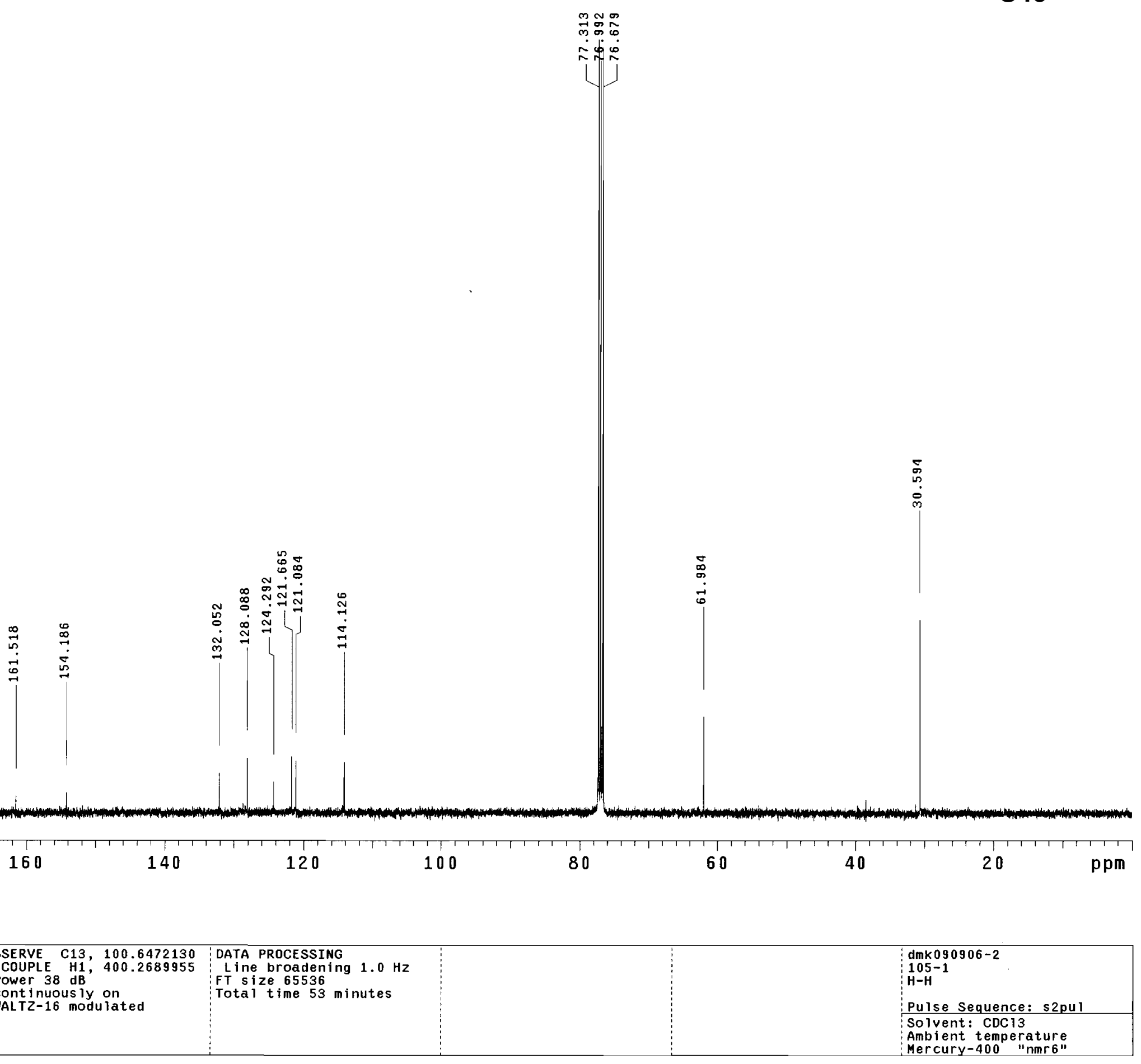
PULSE SEQUENCE
Re lax. de lay 1.000 sec
Pulse. 30.0 degrees

Acq time $1.280 \mathrm{sec}$ Width $25188.9 \mathrm{~Hz}$
1400 repetitions
OBSERVE C13, 100.6472130
OECOUPLE H11, 400.2689955

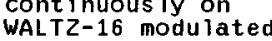

Line broadening $1.0 \mathrm{H}$ al time 53 minutes

$$
\begin{aligned}
& 105-1 \\
& \mathrm{H}-\mathrm{H} \\
& \text { Pulse Sequence } \\
& \text { Solvent: CDC13 }
\end{aligned}
$$

Ambient temperature 

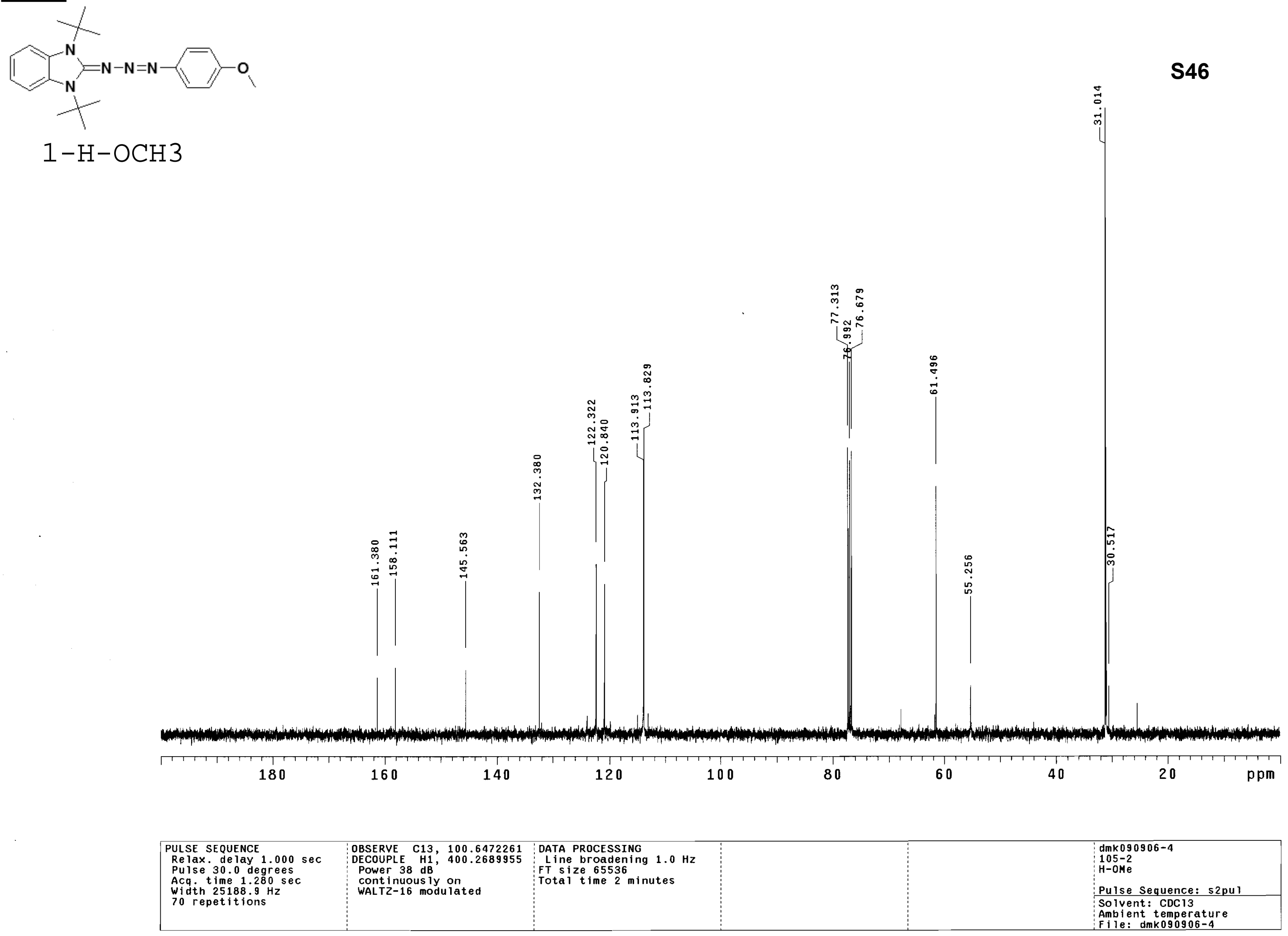
$\left[{ }_{\mathrm{N}}^{\mathrm{N}}=\mathrm{N}-\mathrm{N}=\mathrm{N} \longrightarrow \mathrm{O}\right.$

\section{$1-\mathrm{H}-\mathrm{OCH} 3$}

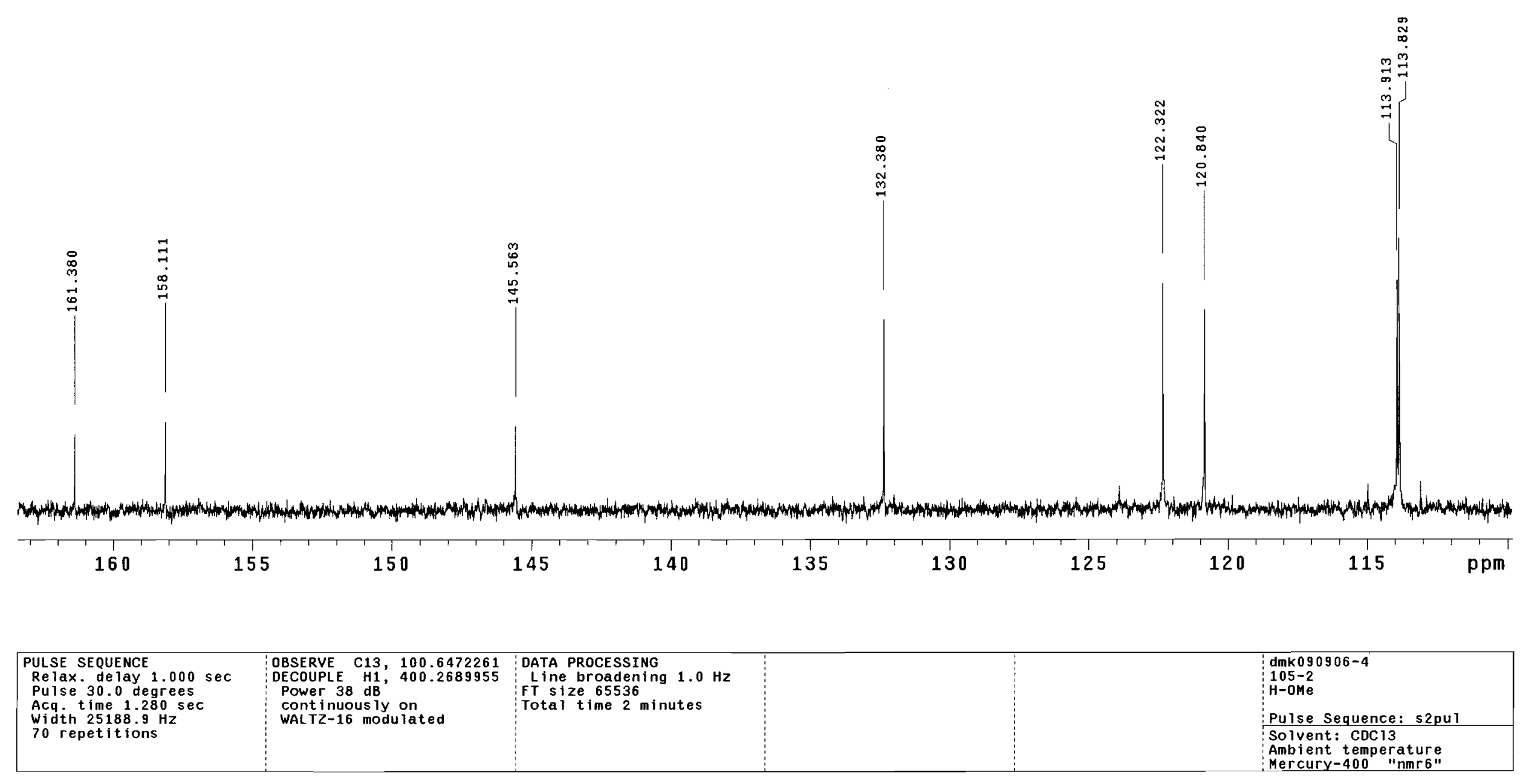



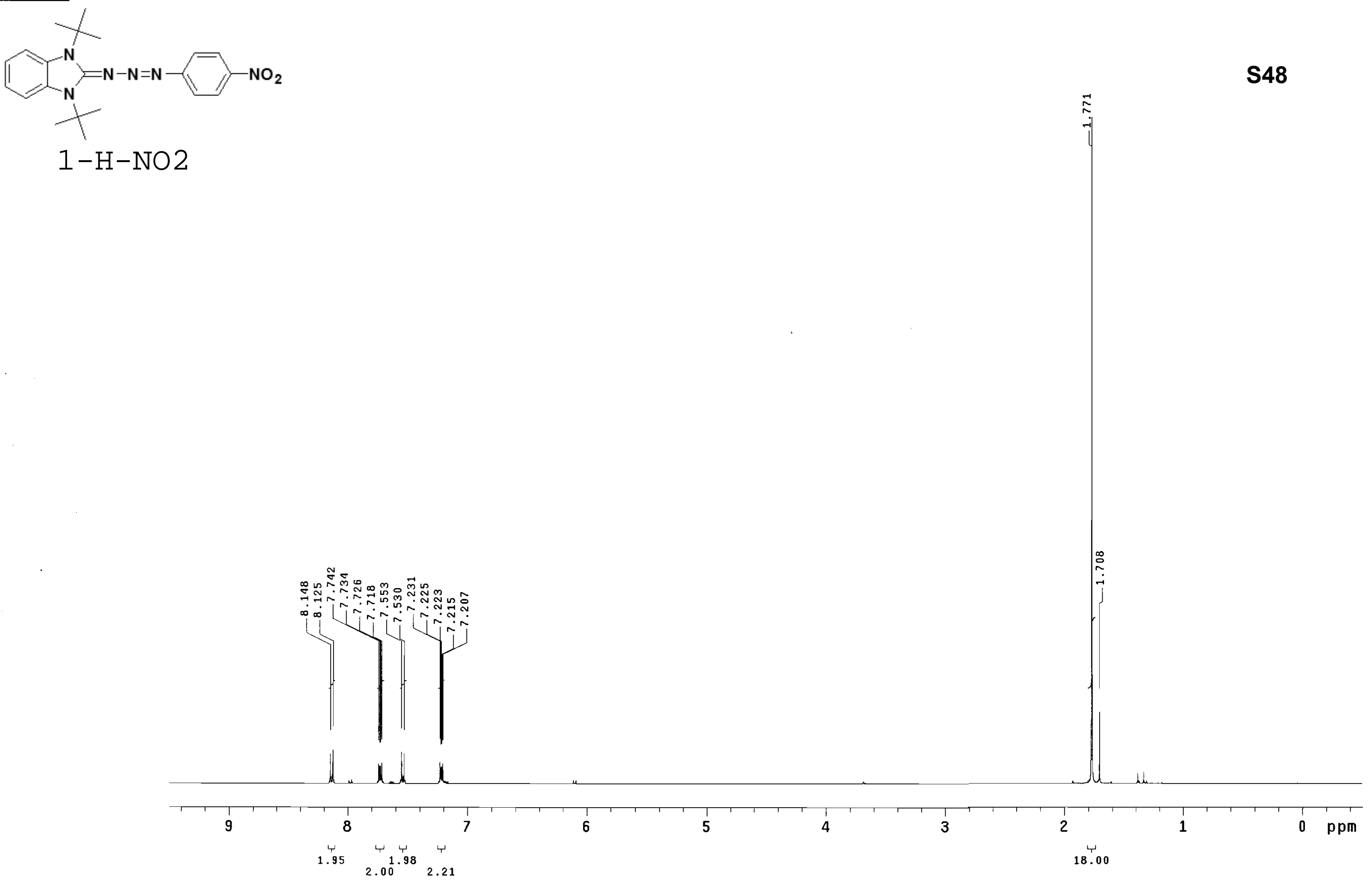

PULSE SEQUENCE
Relax. delay 1.000 sec
Pulse 24.5 degrees
Acq. time 2.856 sec

Acq. t1me 2.856
Width $5602.2 \mathrm{~Hz}$
8 repetitions

$\begin{array}{lll}\text { OBSERVE H1, 400.2669921 DATA PROCESSING } & \end{array}$

Line broadening $0.1 \mathrm{~Hz}$

FT size 32768
Total time 1 minute

$105-3$
$\mathrm{H}-\mathrm{NO2}$

Pulse Sequence: s2pul

Solvent: $\operatorname{CDC} 13$

Ambient temperature 

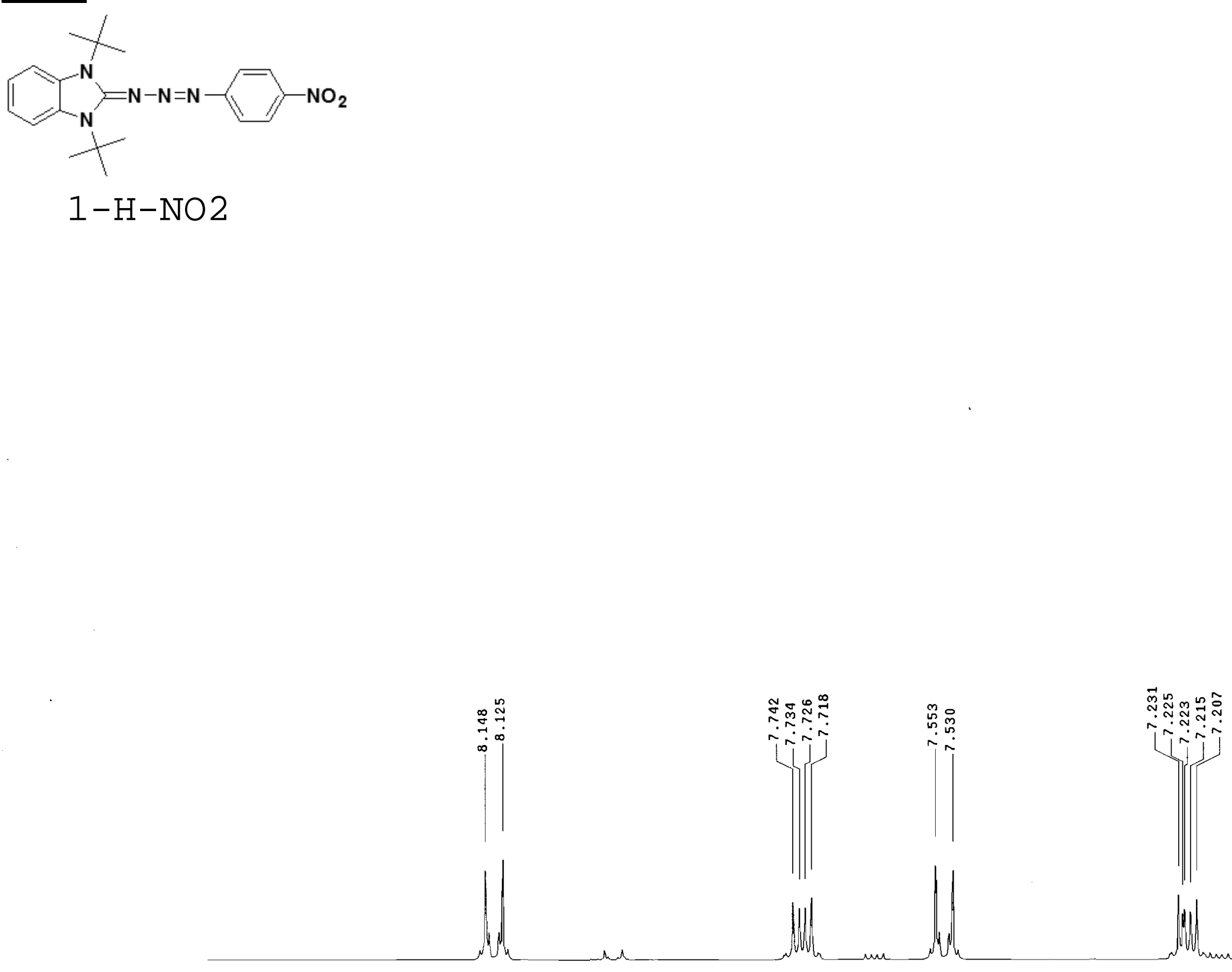

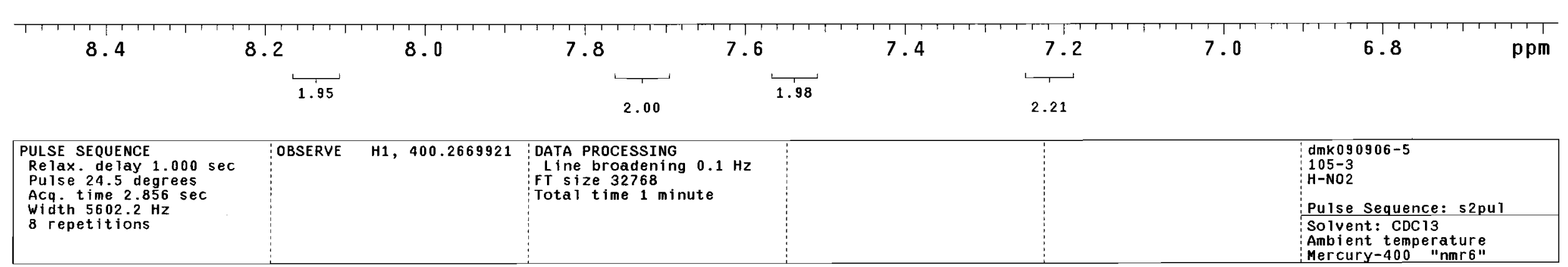




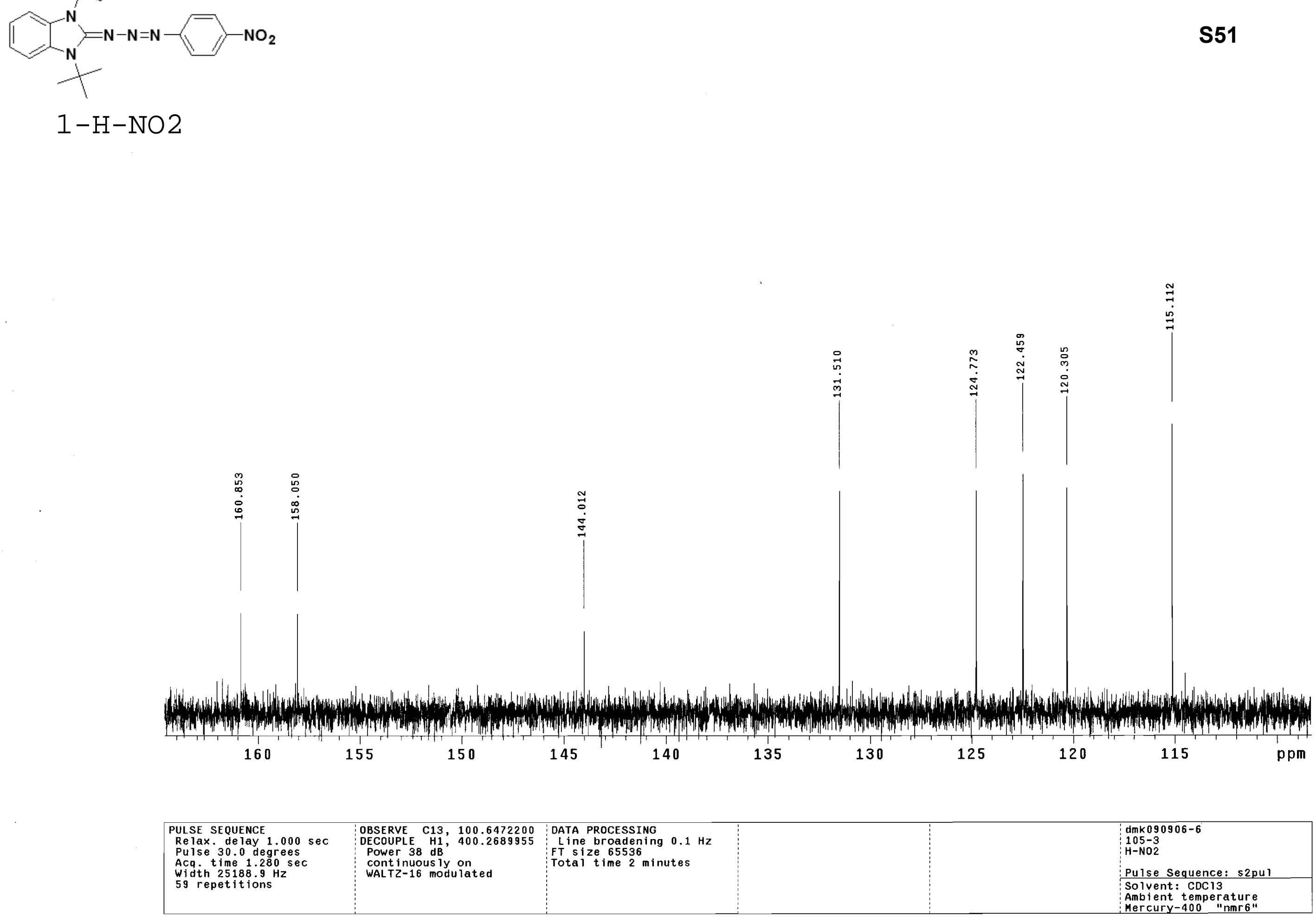

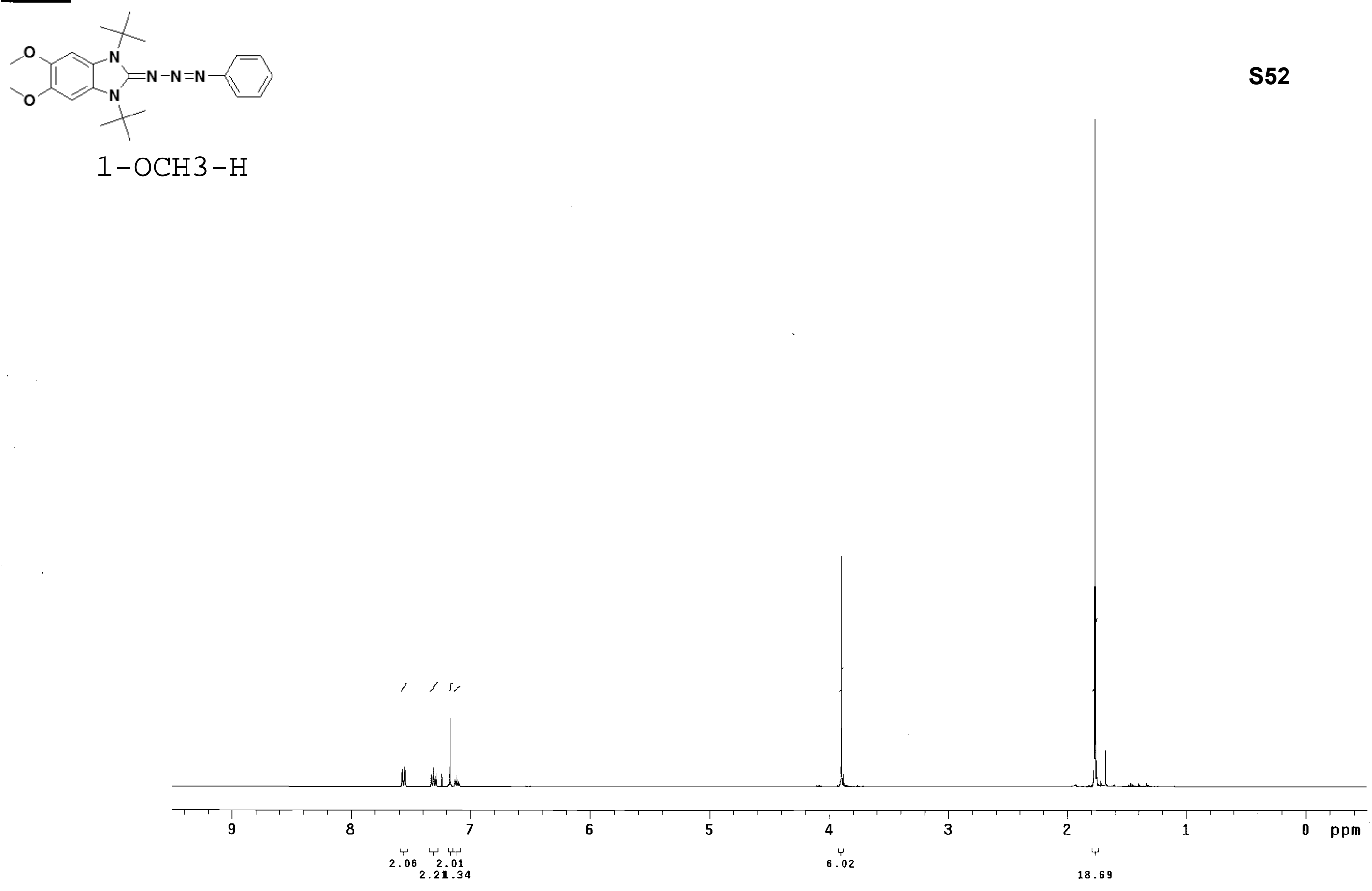

PULSE SEQUENCE
Relax. de lay 1.000 sec

Relax. de lay 1.000 sec
Pulse 24.5 degrees

Acq time 2.856 se
width $5602.2 \mathrm{~Hz}$

7 repetitions
OBSERVE H1, 400.2669859 DATA PROCESSING

Line broadening $0.1 \mathrm{~Hz}$

FT size 32768
Total time 1 minute dmk090906-7 $106-1$
$2 \mathrm{MeO}-\mathrm{H}$

Pulse Sequence: s2pu Solvent: $\operatorname{CDC13}$ Ambient temperature 


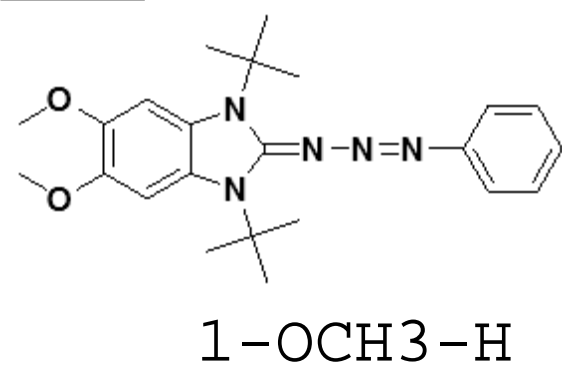

\section{S54}

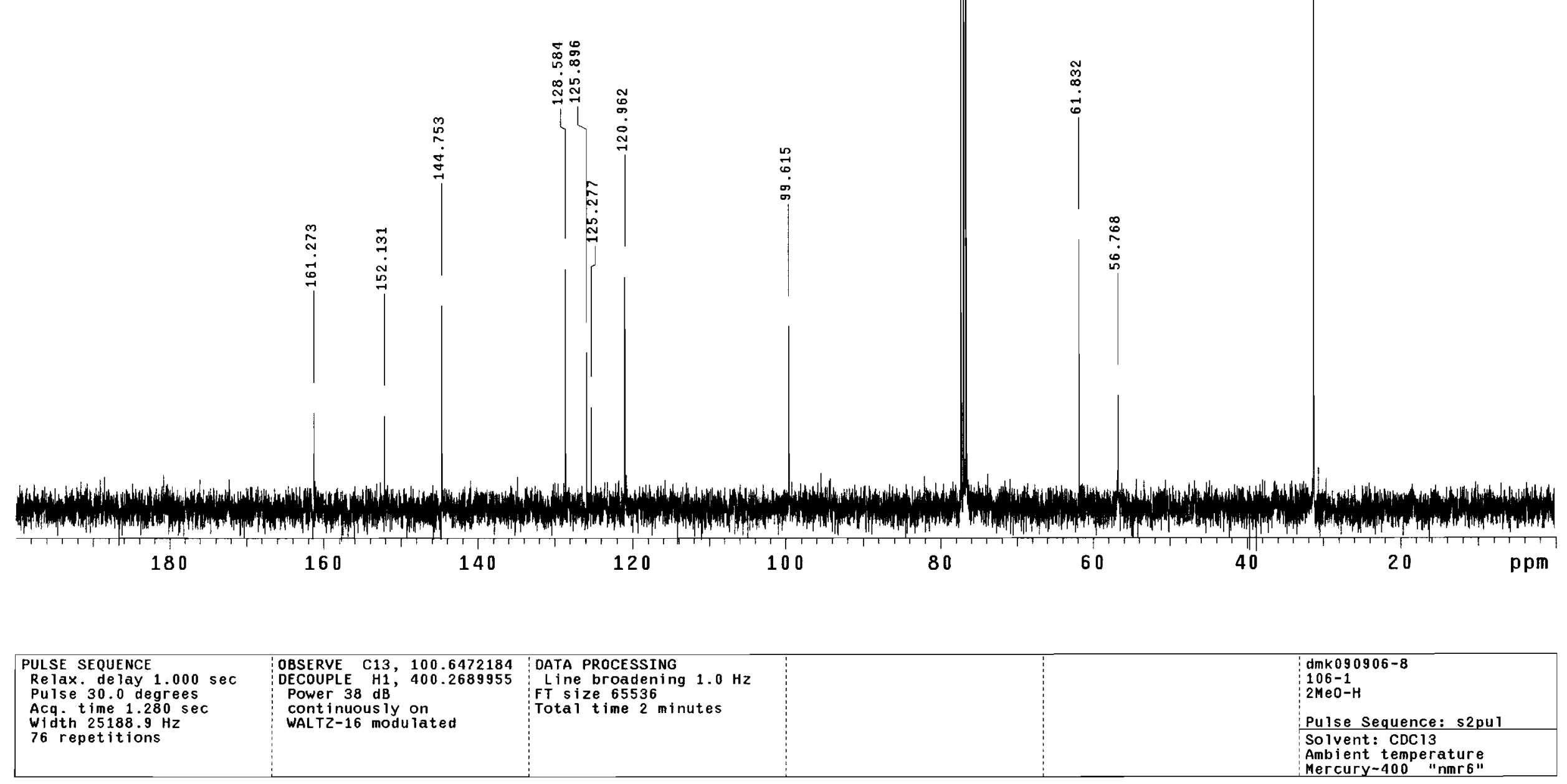




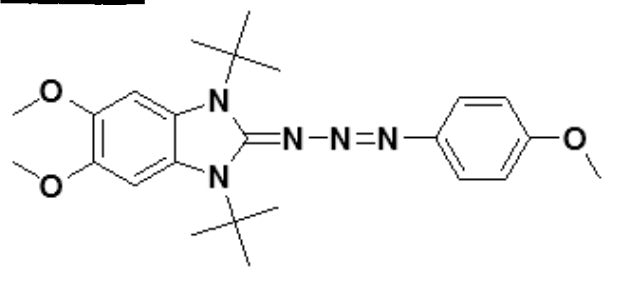

$1-\mathrm{OCH} 3-\mathrm{OCH} 3$

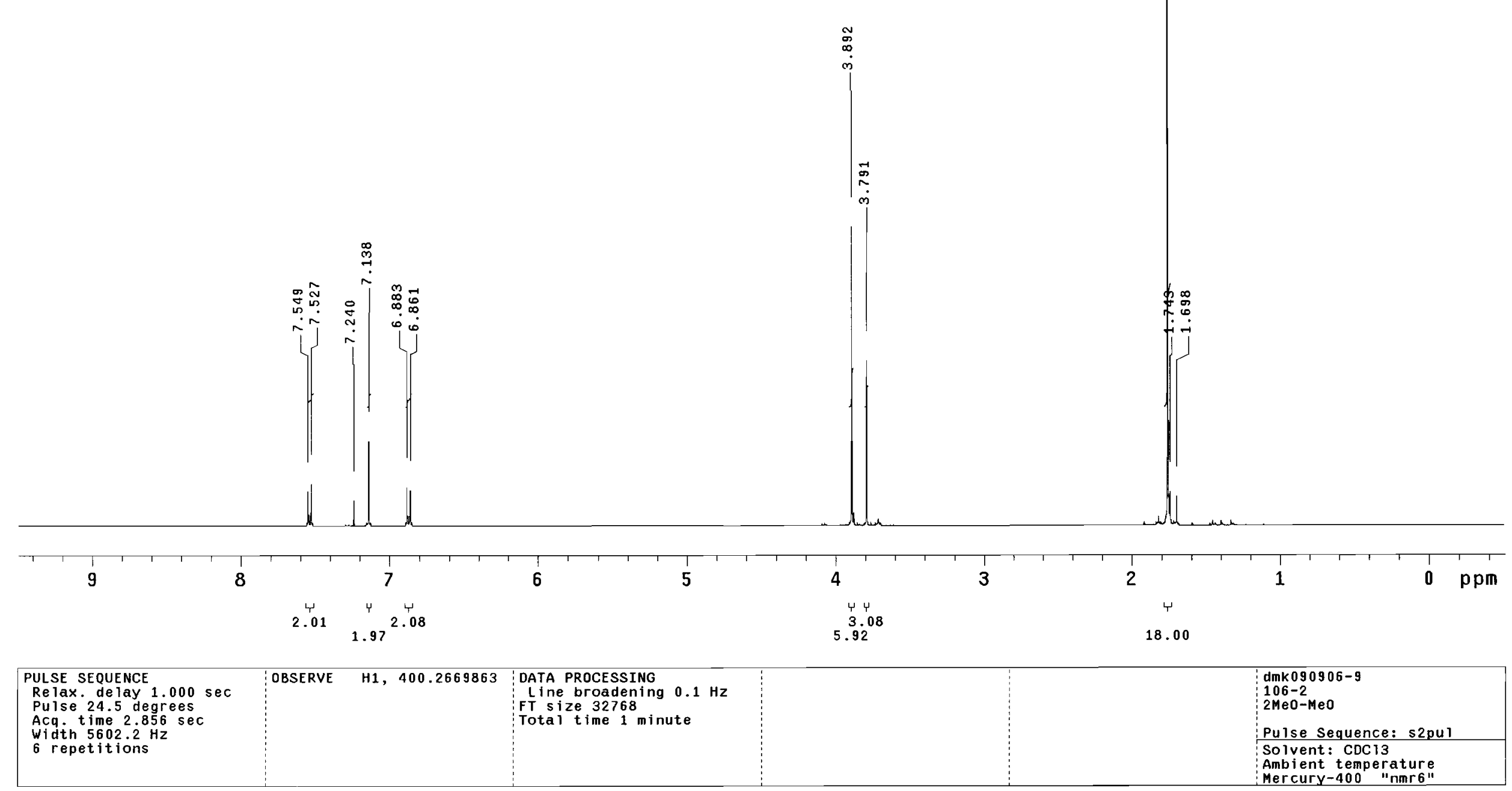



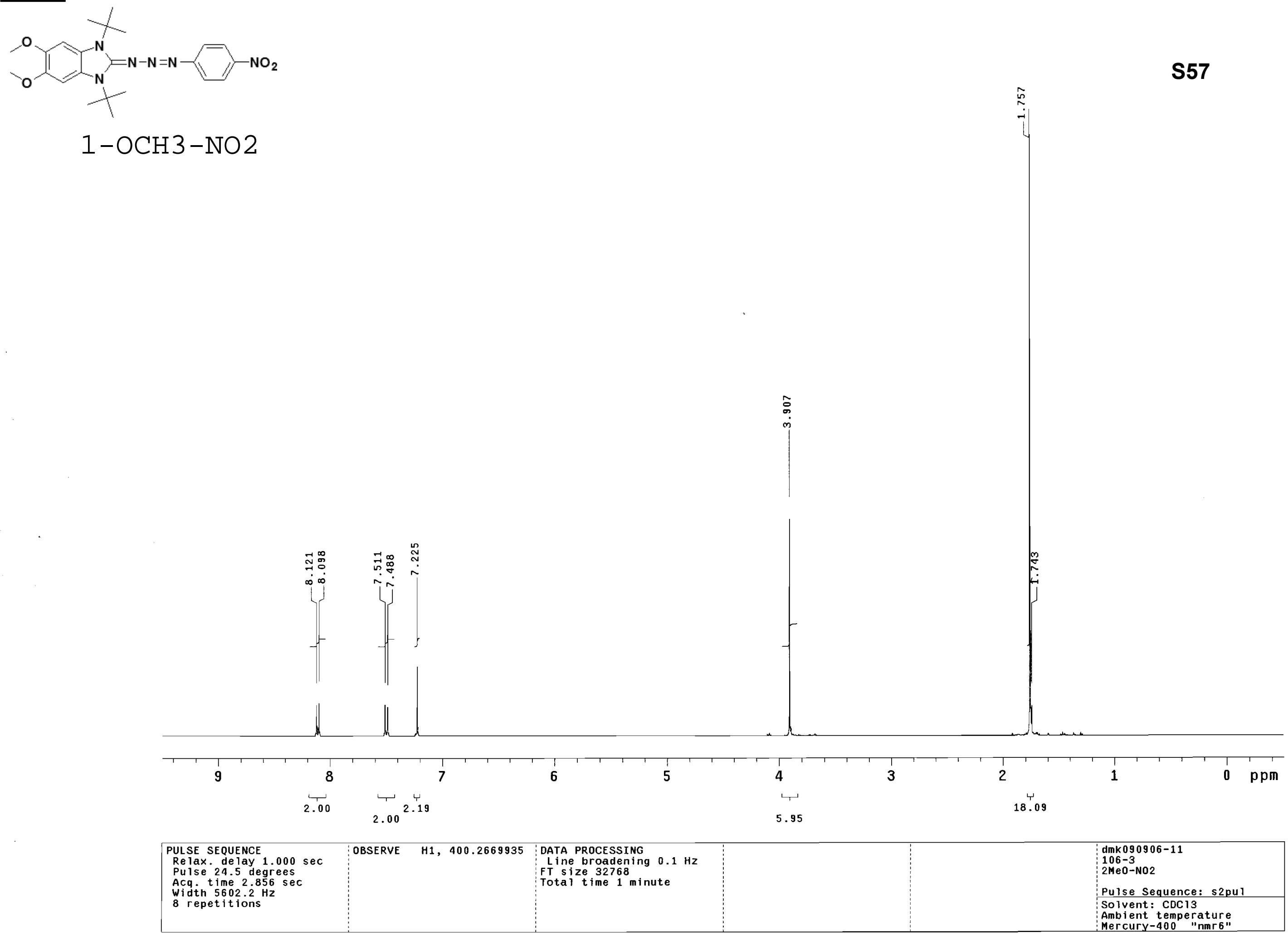

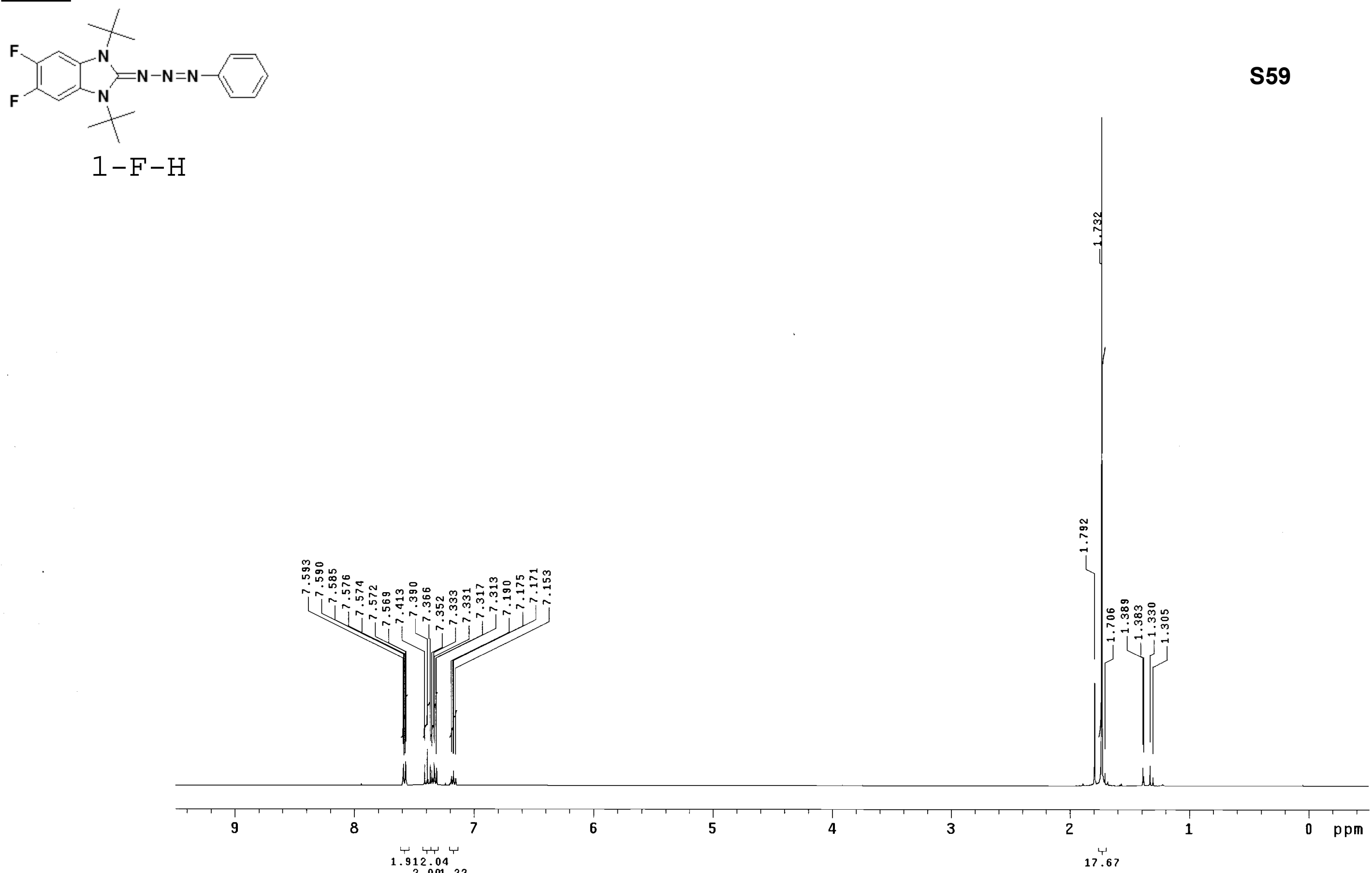

\begin{tabular}{|c|c|c|c|}
\hline $\begin{array}{l}\text { PULSE SEQUENCE } \\
\text { Relax. delay } 1.000 \mathrm{sec} \\
\text { Pulse } 30.0 \text { degrees } \\
\text { Acq. time } 4.049 \text { sec } \\
\text { width } 6410.3 \mathrm{~Hz} \\
\text { 64 repetitions }\end{array}$ & OBSERVE H1, 399.8047198 & $\begin{array}{l}\text { DATA PROCESSING } \\
\text { Line broadening } 0.1 \mathrm{~Hz} \\
\text { FT size } 65536 \\
\text { Total time } 5 \text { minutes }\end{array}$ & $\begin{array}{l}\text { dmk092706-5 } \\
2 F-H \\
\text { Archive directory: } \\
\text { Sample directory: } \\
\text { Pulse Sequence: s2pul } \\
\text { Senkendz gdol }\end{array}$ \\
\hline
\end{tabular}




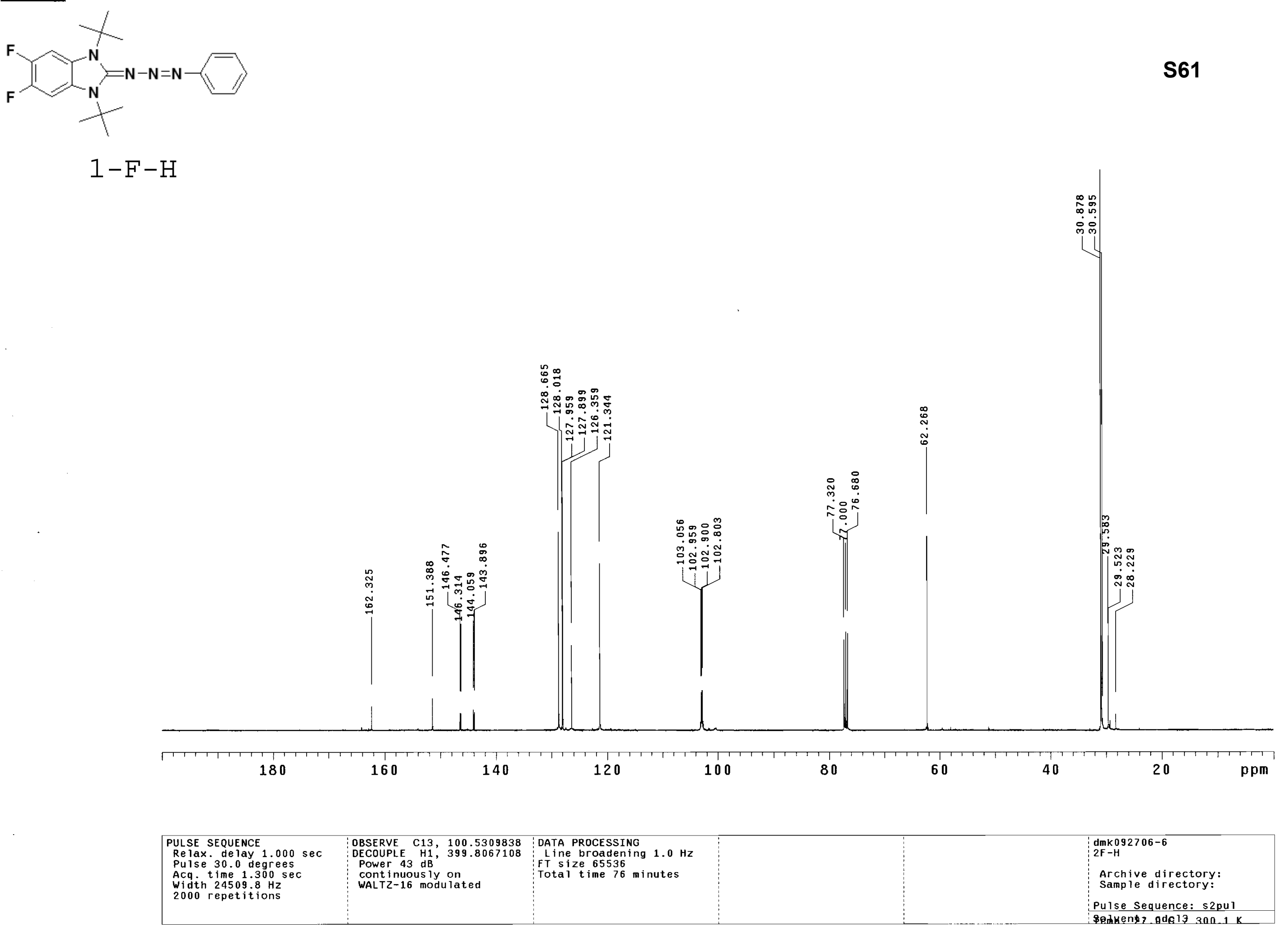




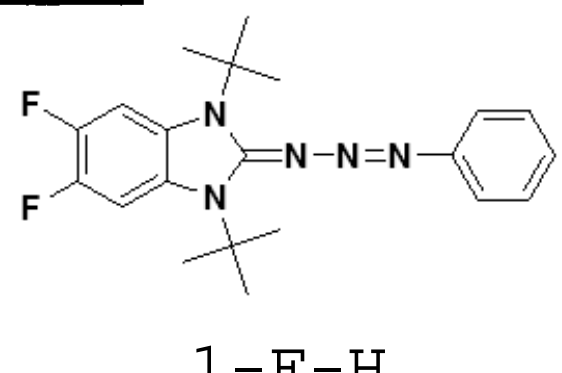

$1-\mathrm{F}-\mathrm{H}$

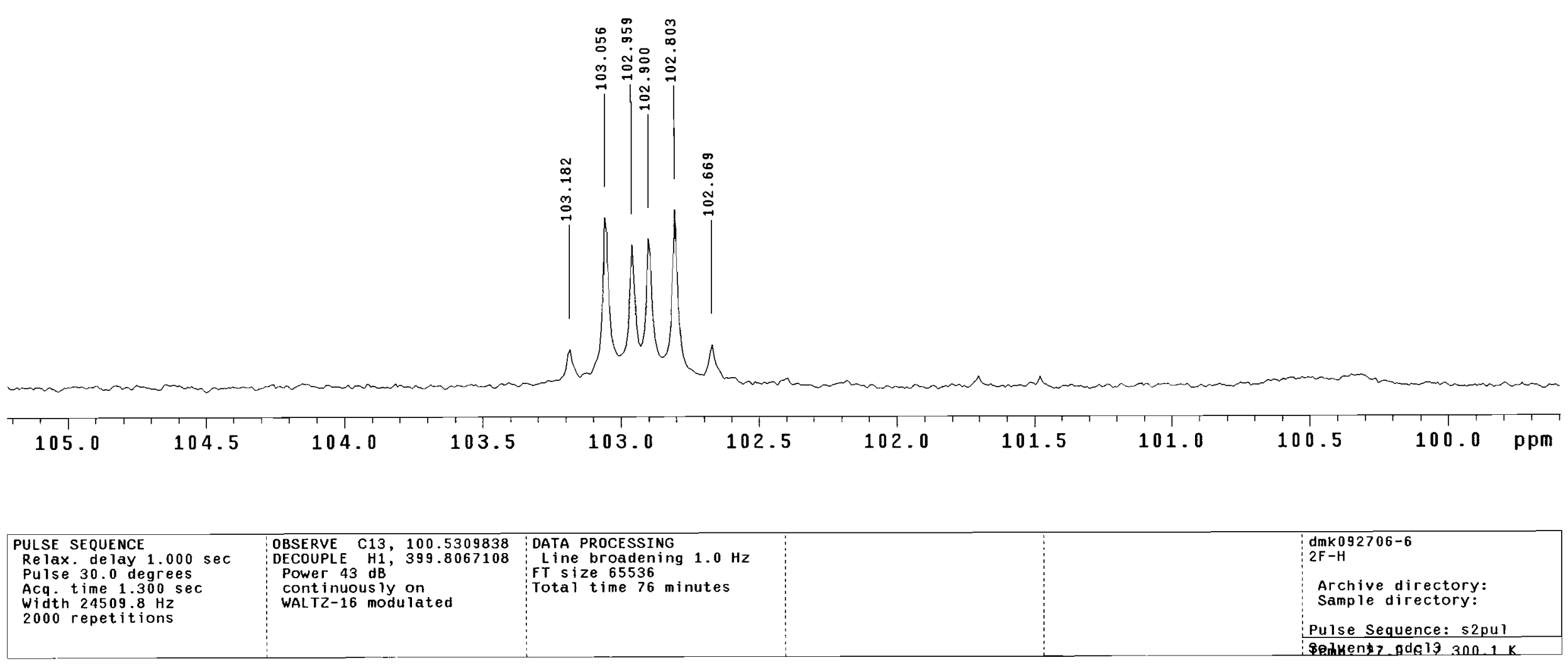



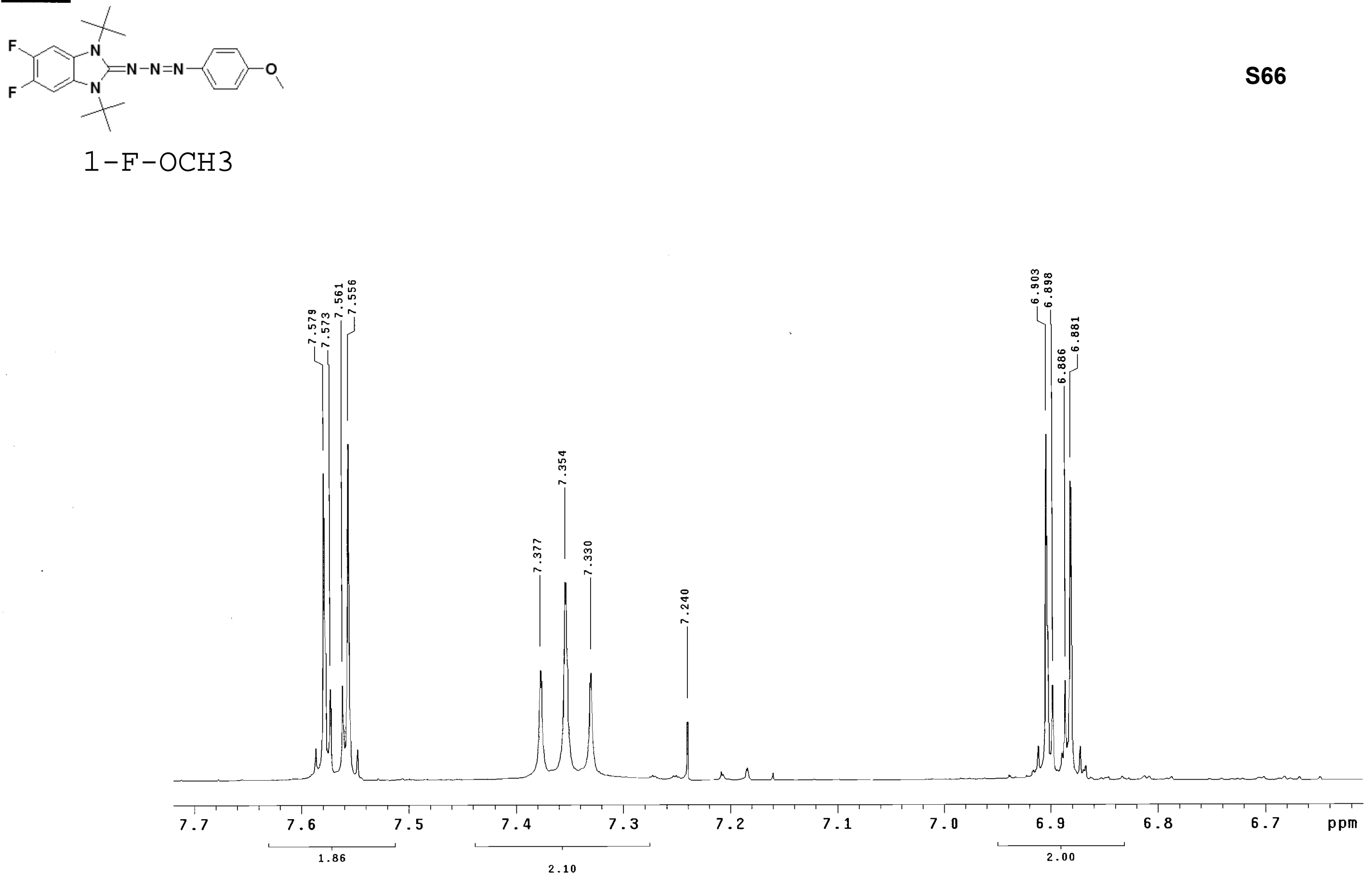

\begin{tabular}{|c|c|c|c|}
\hline $\begin{array}{l}\text { PULSE SEQUENCE } \\
\text { Relax. delay } 1.000 \text { sec } \\
\text { Pulse } 30.0 \text { degrees } \\
\text { Acq. time } 4.049 \text { sec } \\
\text { Width } 6410.3 \mathrm{~Hz} \\
64 \text { repetitions }\end{array}$ & OBSERVE $\quad \mathrm{H1}, 399.8047194$ & $\begin{array}{l}\text { DATA PROCESSING } \\
\text { Line broadening } 0.1 \mathrm{~Hz} \\
\text { FT size } 65536 \\
\text { Total time } 5 \text { minutes }\end{array}$ & $\begin{array}{l}\text { dmk092606-3 } \\
2 \mathrm{~F}-\mathrm{MeO} \\
\text { Archive directory: } \\
\text { Sample directory: } \\
\text { Pulse Sequence: s2pul }\end{array}$ \\
\hline
\end{tabular}




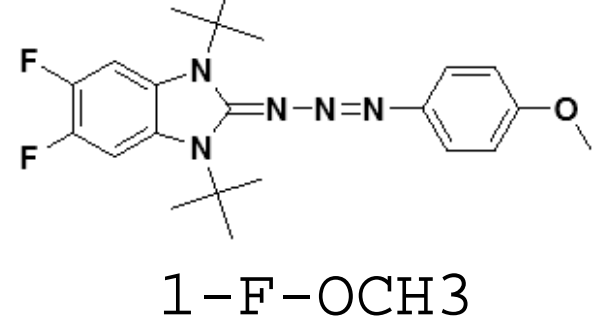

$1-\mathrm{F}-\mathrm{OCH} 3$

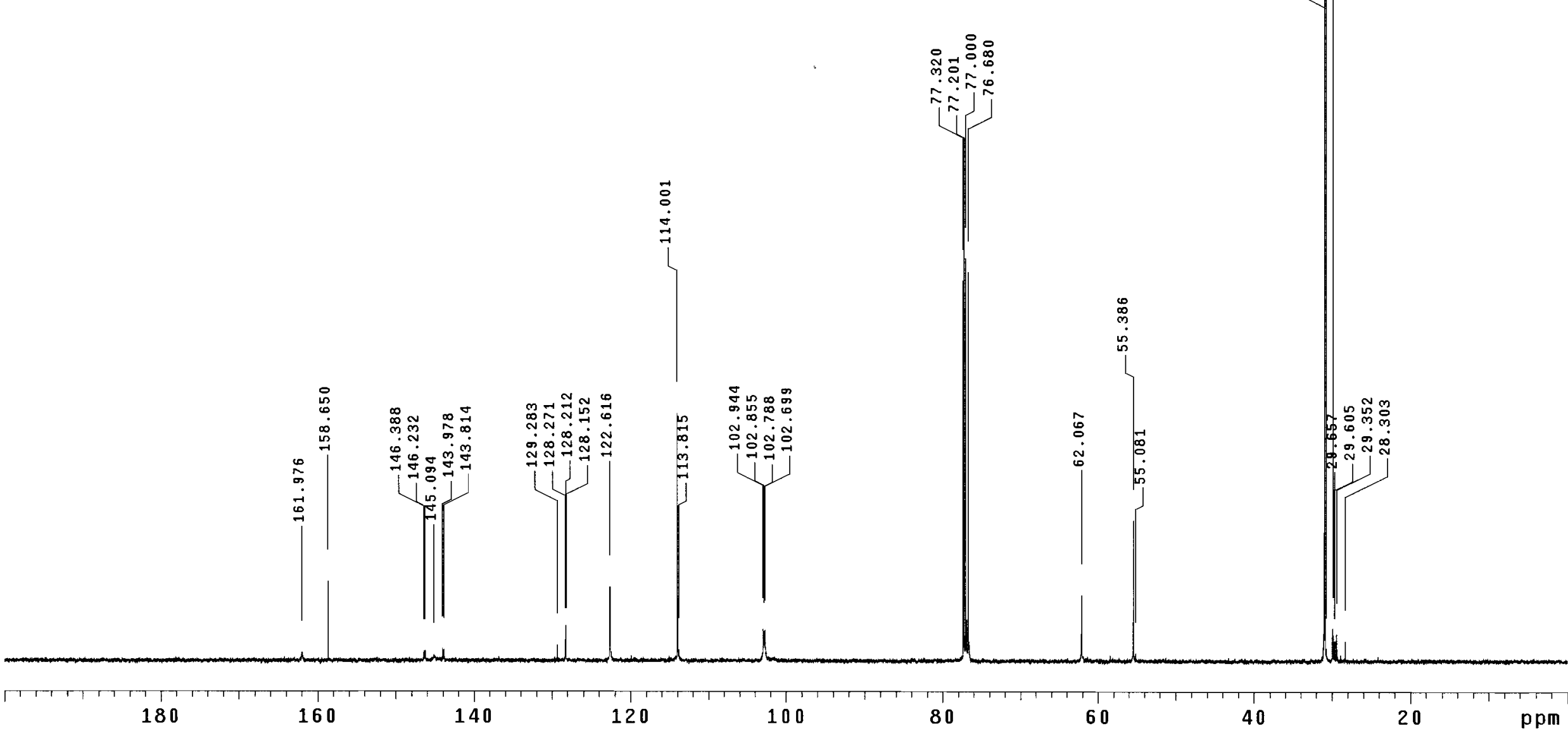

\begin{tabular}{|c|c|c|c|}
\hline $\begin{array}{l}\text { PULSE SEQUENCE } \\
\text { Re lax. de lay } 1.000 \mathrm{sec} \\
\text { Pulse } 30.0 \text { degrees } \\
\text { Acq time } 1.300 \mathrm{sec} \\
\text { width } 24509.8 \mathrm{~Hz} \\
5000 \text { repetitions }\end{array}$ & $\begin{array}{l}\text { OBSERVE C13, } 100.5309770 \\
\text { DECOUPLE H1, } 399.8067108 \\
\text { POWer 43 dB } \\
\text { cont inuously on } \\
\text { WALTZ-16 modulated } \\
\text { WA }\end{array}$ & $\begin{array}{l}\text { DATA PROCESSING } \\
\text { Line broadening } 1.0 \mathrm{~Hz} \\
\text { sT sze } 65536 \\
\text { Total time } 3.2 \text { hours }\end{array}$ & $\begin{array}{l}\text { dmk092706-4 } \\
2 F-0 M e \\
\text { Archive directory: } \\
\text { Sample directory: } \\
\text { Pulse Sequence: s2pul } \\
\text { Pedmendz gdgla } 300.1 \mathrm{k}\end{array}$ \\
\hline
\end{tabular}




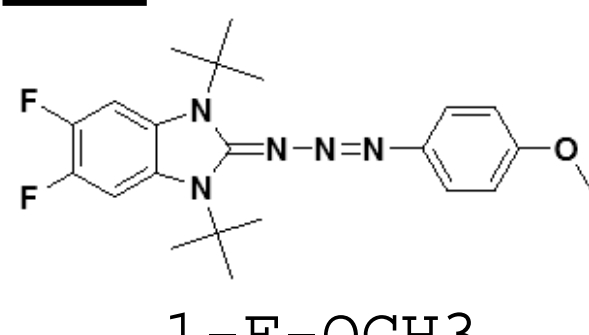

1-F-OCH3

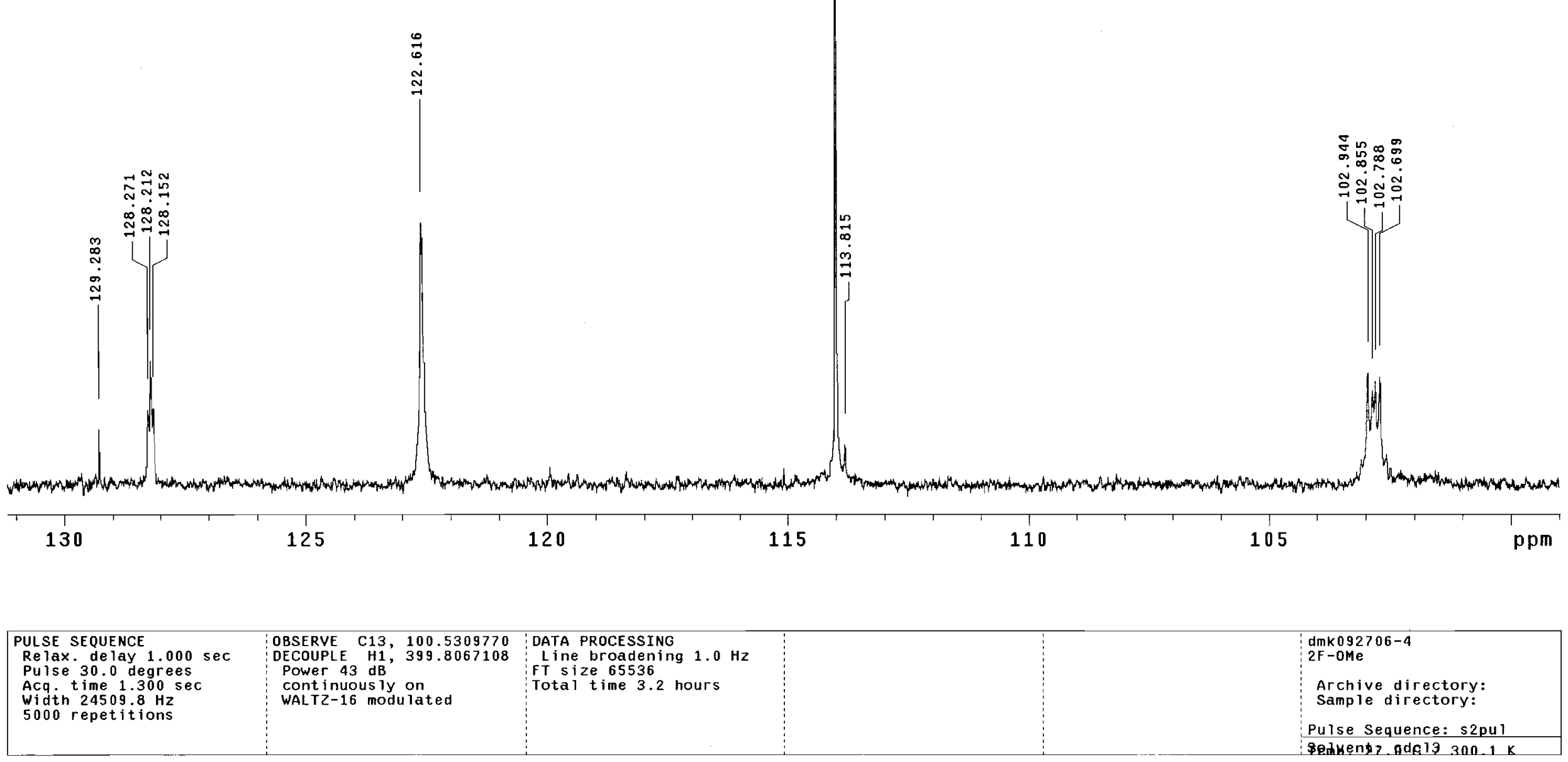


$1-\mathrm{F}-\mathrm{NO} 2$

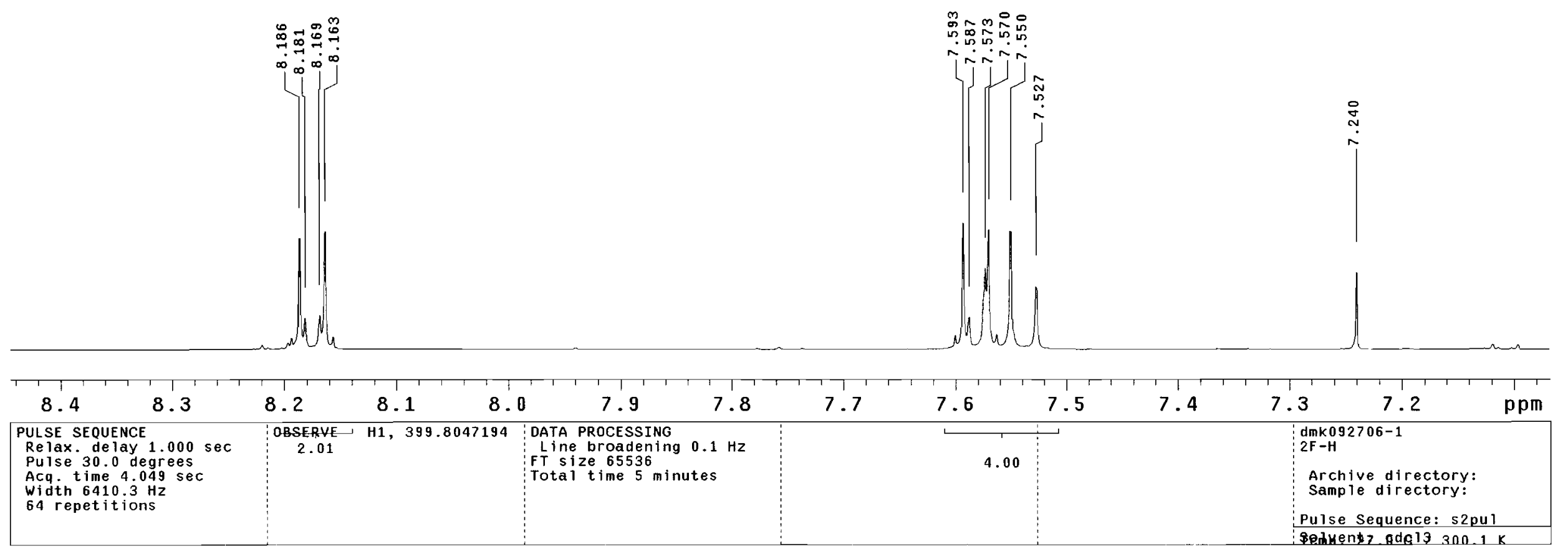




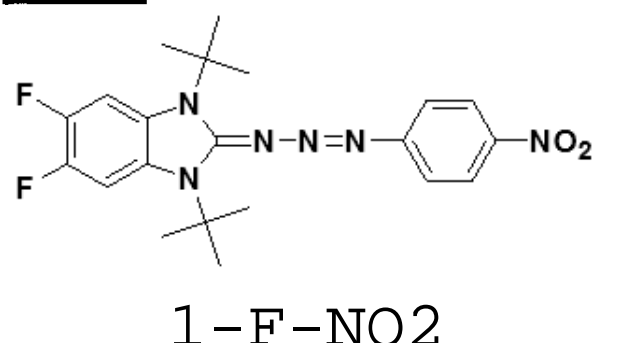

$1-\mathrm{F}-\mathrm{NO} 2$

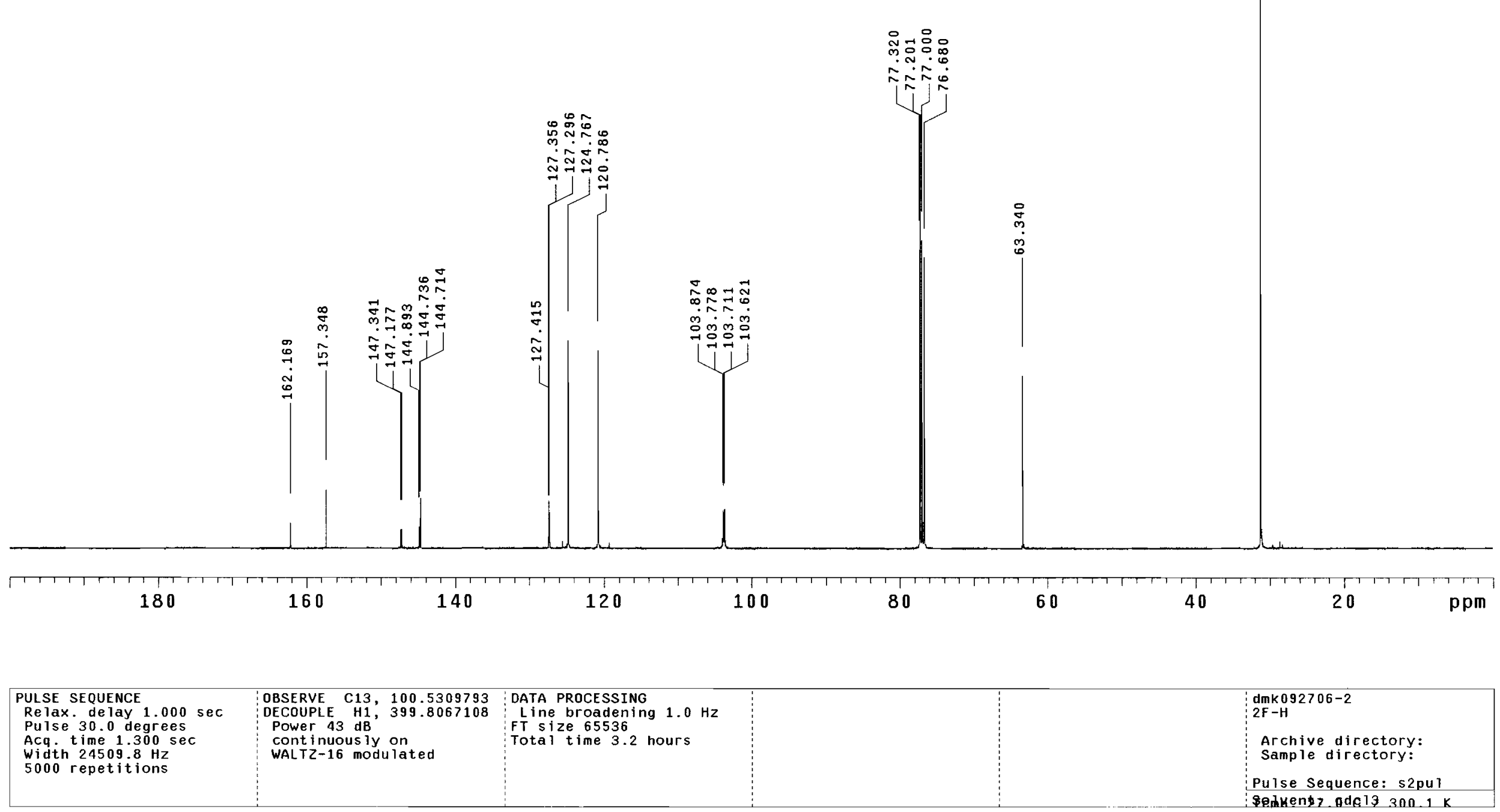




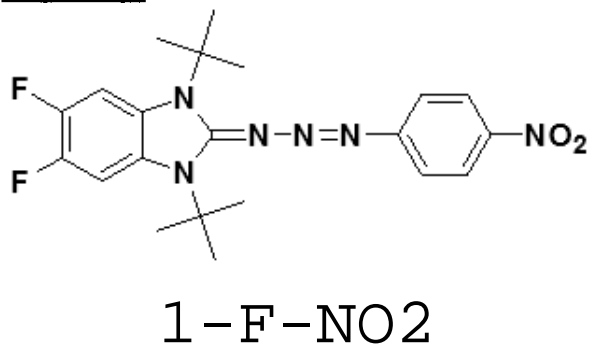

$1-\mathrm{F}-\mathrm{NO} 2$

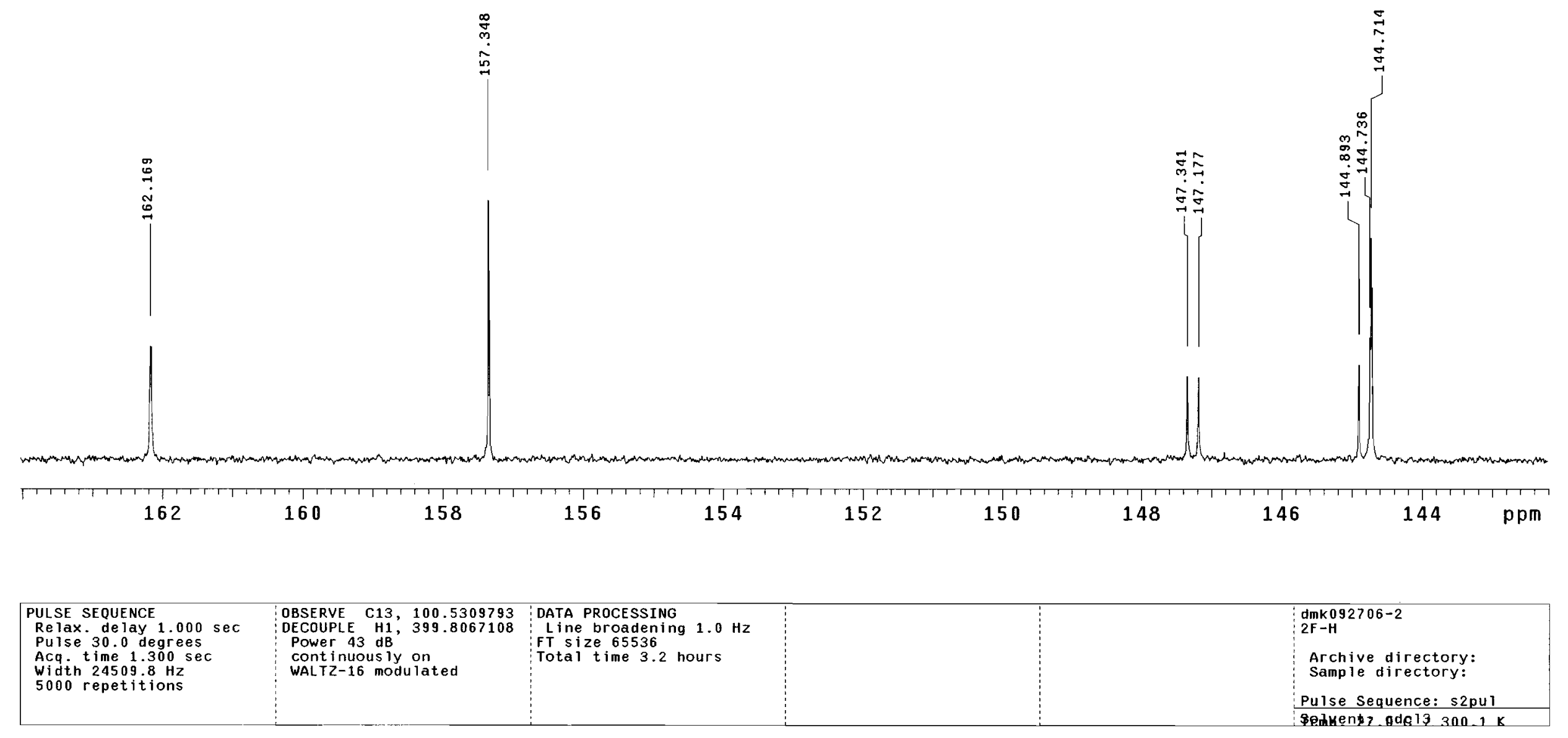




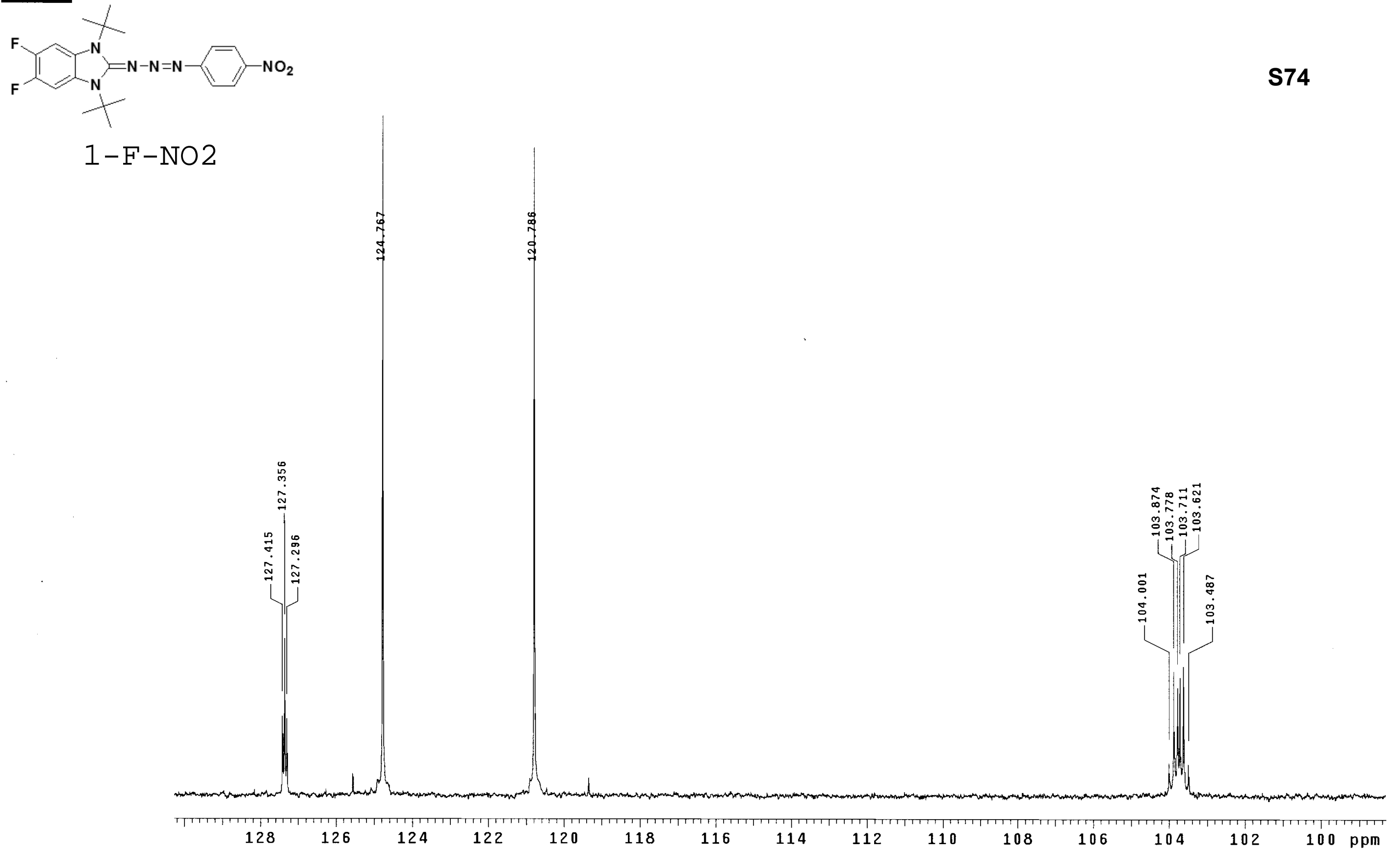

\begin{tabular}{|c|c|c|c|}
\hline $\begin{array}{l}\text { PULSE SEQUENCE } \\
\text { Relax. de lay } 1.000 \mathrm{sec} \\
\text { Pulse } 30.0 \text { degrees } \\
\text { Acq. time } 1.300 \mathrm{sec} \\
\text { Width } 24509.8 \mathrm{~Hz} \\
5000 \text { repetitions }\end{array}$ & $\begin{array}{l}\text { OBSERVE C13, } 100.5309793 \\
\text { DECOUPLE H1, } 399.8067108 \\
\text { POWer 43 dB } \\
\text { cont inuous iy on } \\
\text { WALTZ-16 modulated }\end{array}$ & $\begin{array}{l}\text { DATA PROCESSING } \\
\text { Line broadening } 1.0 \mathrm{~Hz} \\
\text { FT size } 65536 \\
\text { Total time } 3.2 \text { hours }\end{array}$ & $\begin{array}{l}\text { dmk092706-2 } \\
2 \mathrm{~F}-\mathrm{H} \\
\text { Archive directory: } \\
\text { Sample directory: } \\
\text { Pulse Sequence: s2pul } \\
\text { Pedmenter Bdgl } 300.1 \mathrm{~K}\end{array}$ \\
\hline
\end{tabular}




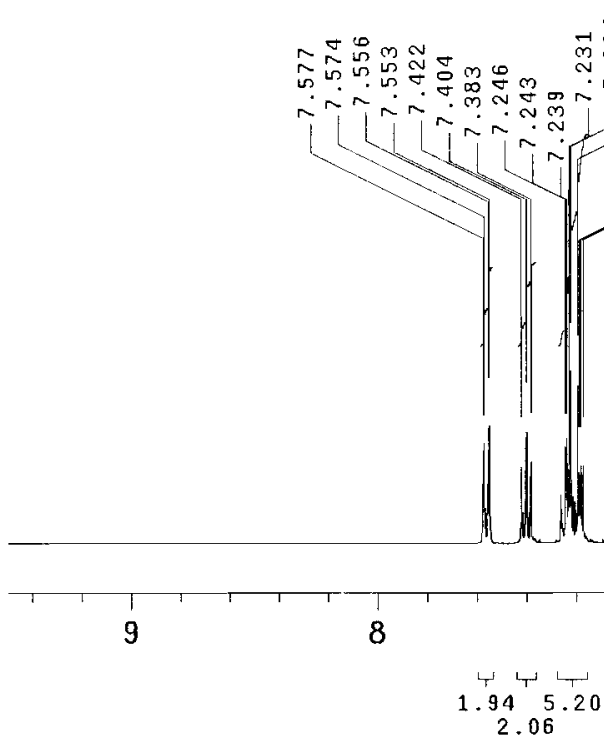

PULSE SEQUENCE Relax. delay $1.000 \mathrm{sec}$ Acq. time $2.856 \mathrm{sec}$ width $5602.2 \mathrm{HZ}$ repetitions
OBSERVE H1, 400.2677522 DATA PROCESSING Line broadening $0.1 \mathrm{~Hz}$ Total time 1 minute Total time 1 minute

dmk101906-5

Pulse Sequence: s2pul

Solvent: $\mathrm{cd} 2 \mathrm{c} 12$

Ambient temperature 


$$
3-\mathrm{H}-\mathrm{H}
$$

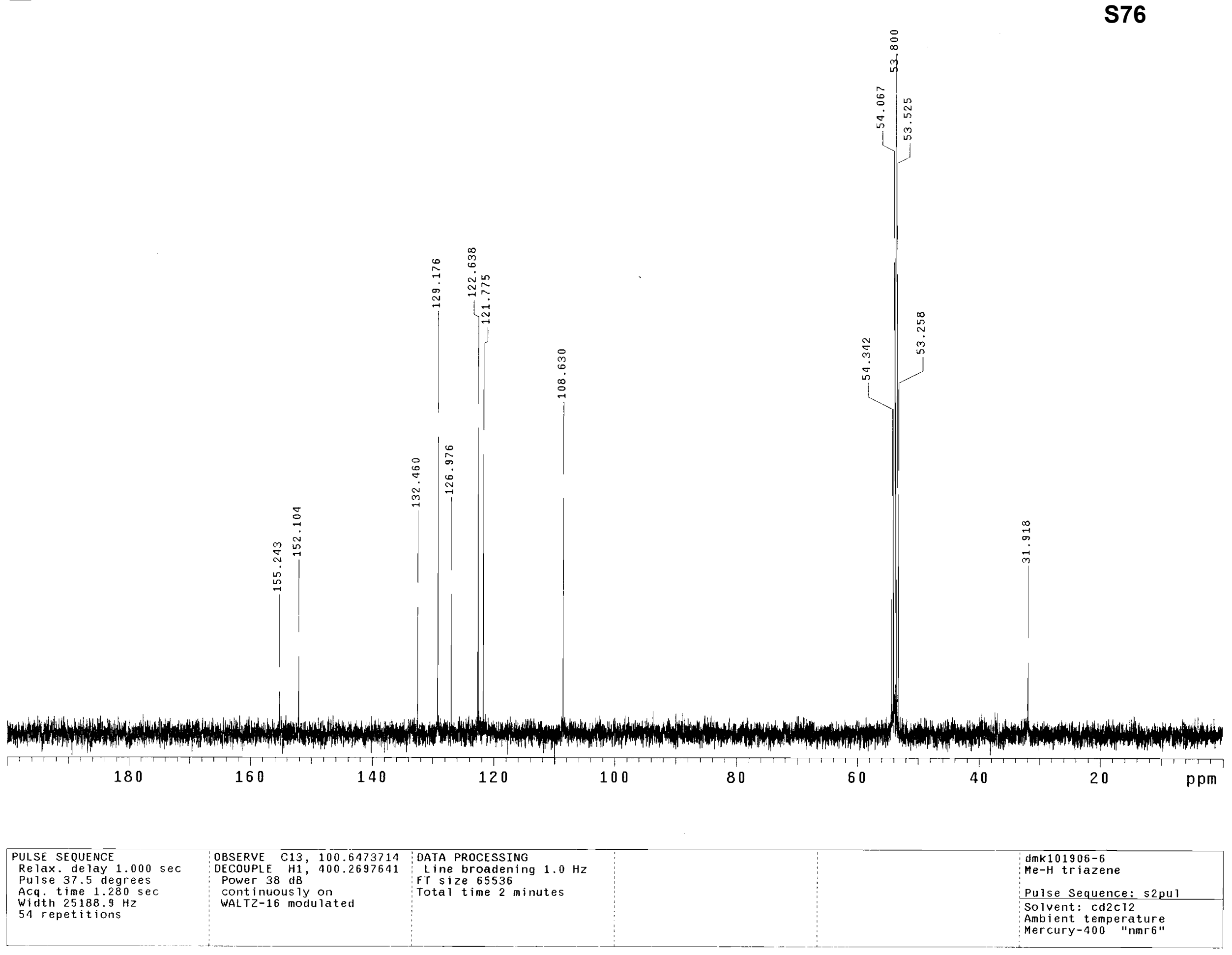




\section{$3-\mathrm{H}-\mathrm{NO} 2$}

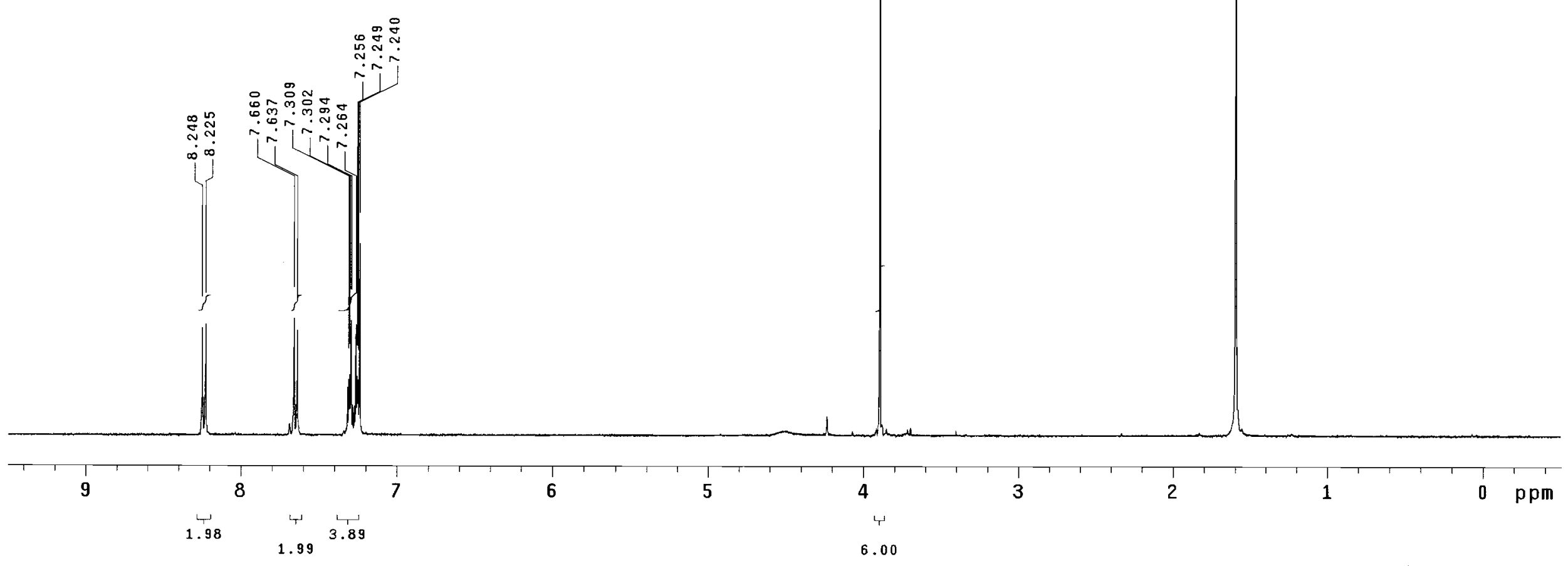

\begin{tabular}{|lll} 
OBSERVE H1, 400.2669866 & DATA PROCESSING \\
Line broadening $0.1 \mathrm{~Hz}$
\end{tabular} FT size 32768
Total time 1 minute

\section{dmk031707-1}

Pulse Sequence: s2pu Solvent: $\mathrm{CDCl} 3$ Ambient temperatur
Acq. time 32.7 degrees

Width $5602.2 \mathrm{~Hz}$
2 repetitions 


\section{$3-\mathrm{H}-\mathrm{NO} 2$}

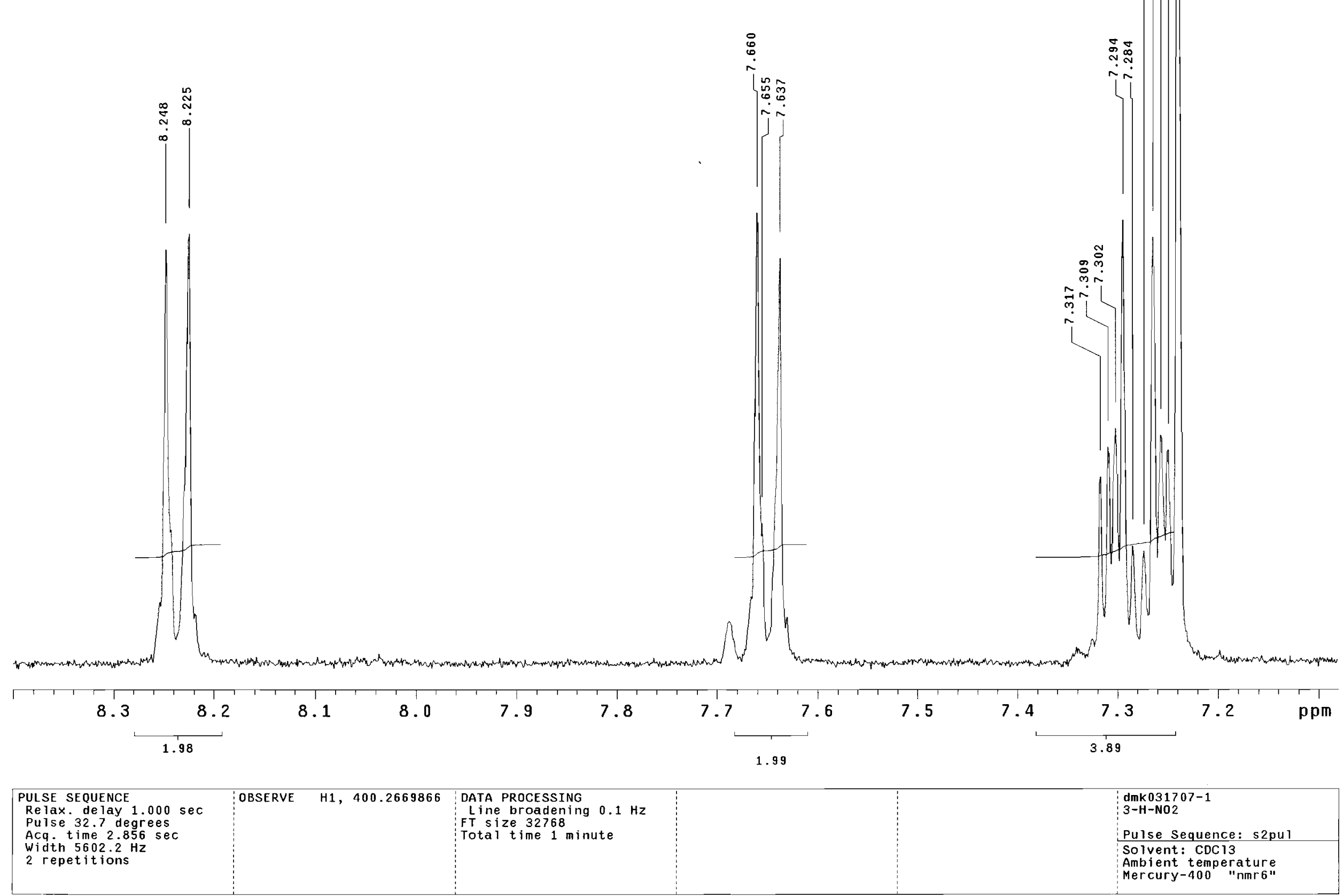


$\overbrace{\mathrm{N}}^{\mathrm{N}} \rightleftharpoons \mathrm{N}-\mathrm{N}=\mathrm{N}-\overrightarrow{\mathrm{NO}_{2}}$

\section{$3-\mathrm{H}-\mathrm{NO} 2$}

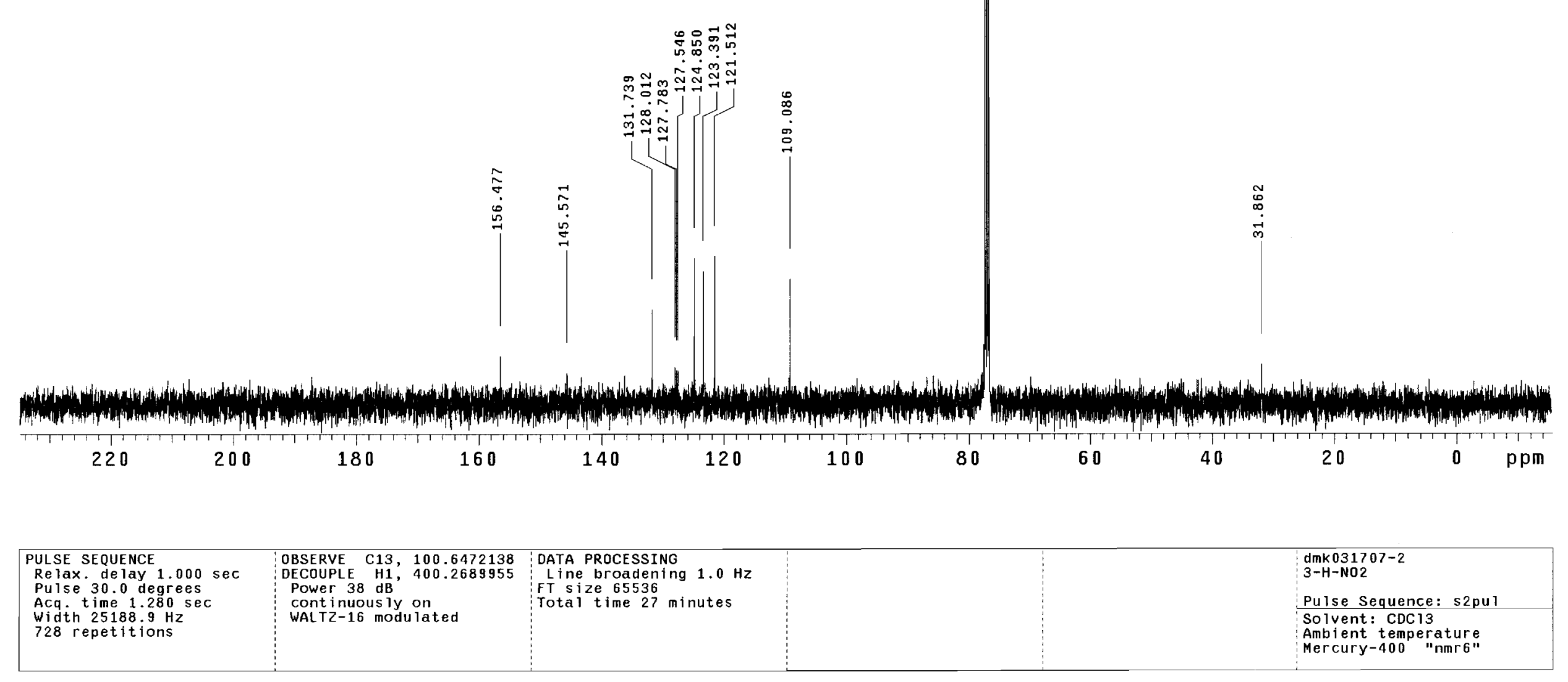


$3-\mathrm{OCH} 3-\mathrm{H}$

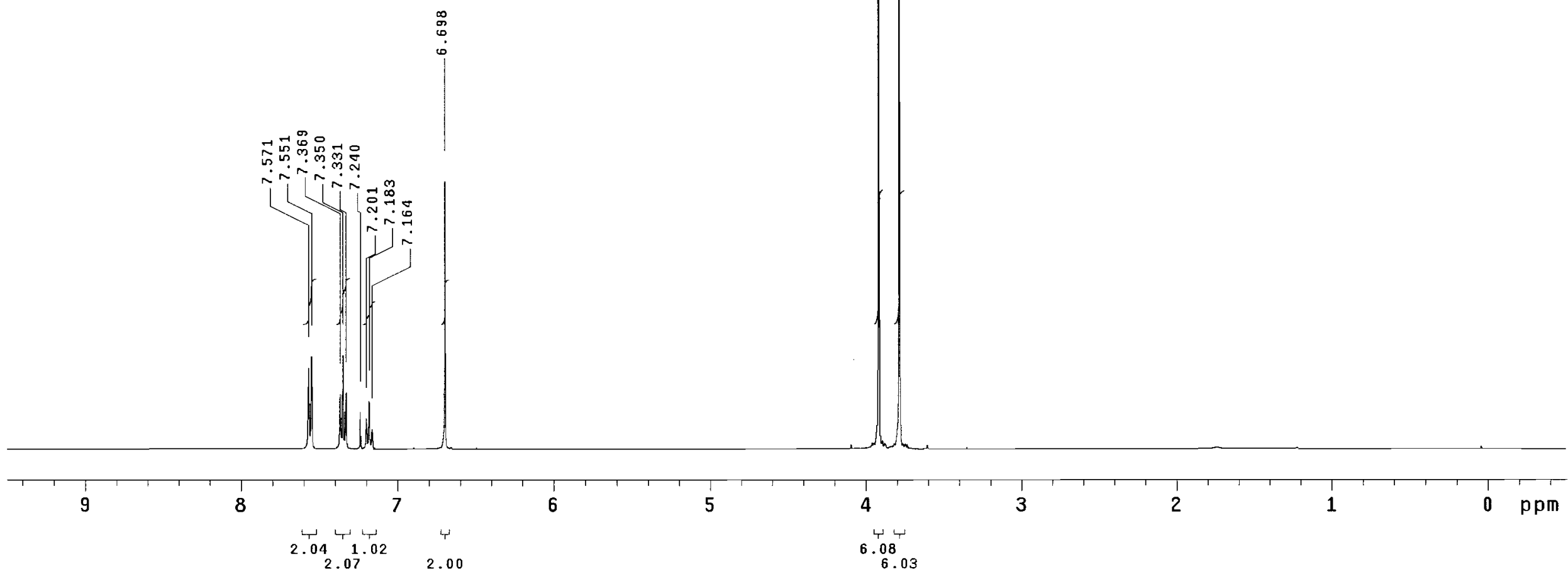




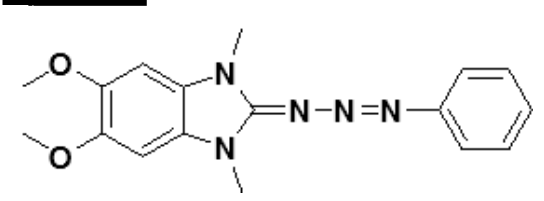

$3-\mathrm{OCH} 3-\mathrm{H}$
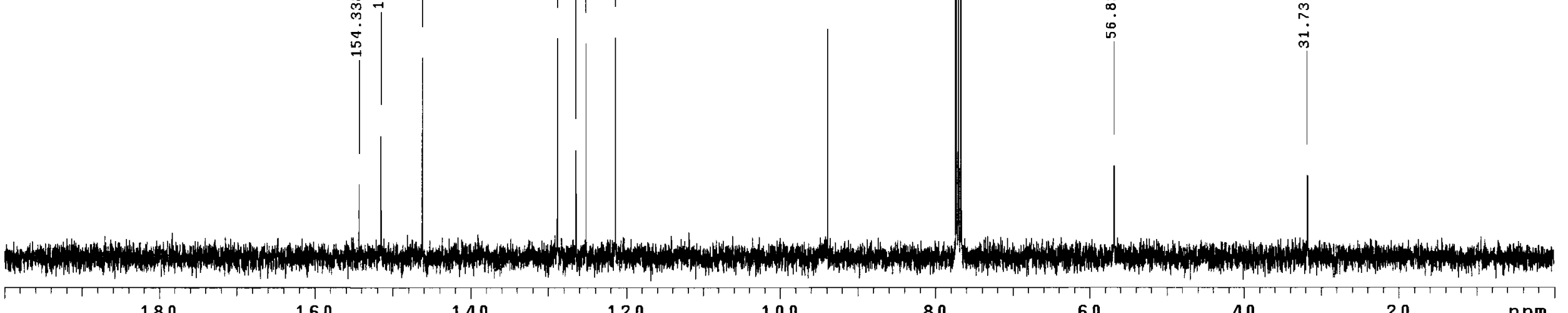

180

160

140

120

100

80

60

40

20

ppm

\begin{tabular}{|l|l|}
\hline PULSE SEQUENCE & OBSERVE C13, 100.6472184 \\
Relax. delay 1.000 sec & OECOUPLE H1, 400.2689955 \\
Pulse 30.0 degrees & POwer 38 dB \\
ACq. time 1.280 sec & COntinuous ly on \\
Width 25188.9 Hz & WALT2-16 modulated \\
124 repetitions &
\end{tabular}
DATA PROCESSING
Line broadenin
Line broadening $1.0 \mathrm{~Hz}$
Total time 4 minutes

Pulse Sequence: s2pul

Solvent: CDC13 Ambient temperature
Mercury-400 "nmr6" 


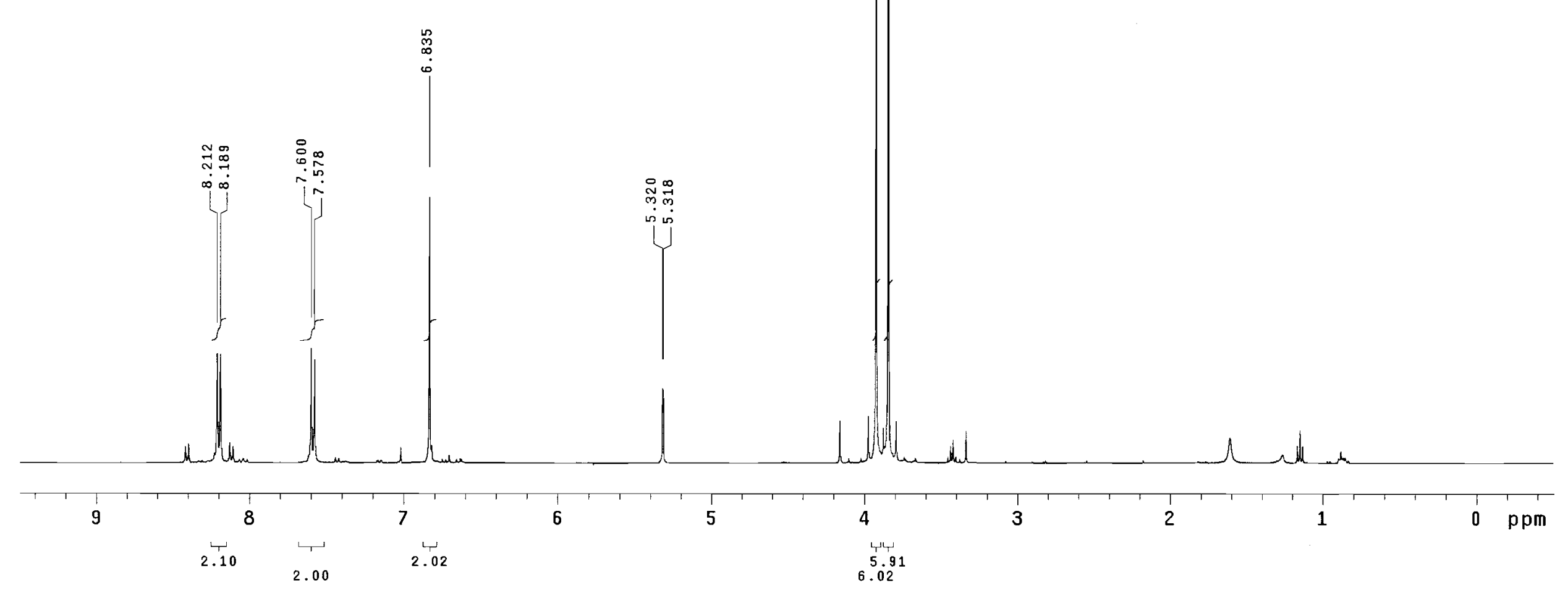

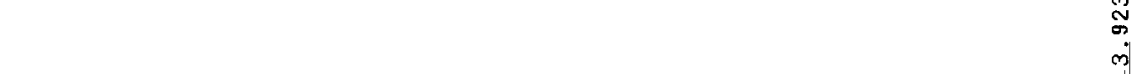




$$
\text { 3-OCH3-NO2 }
$$

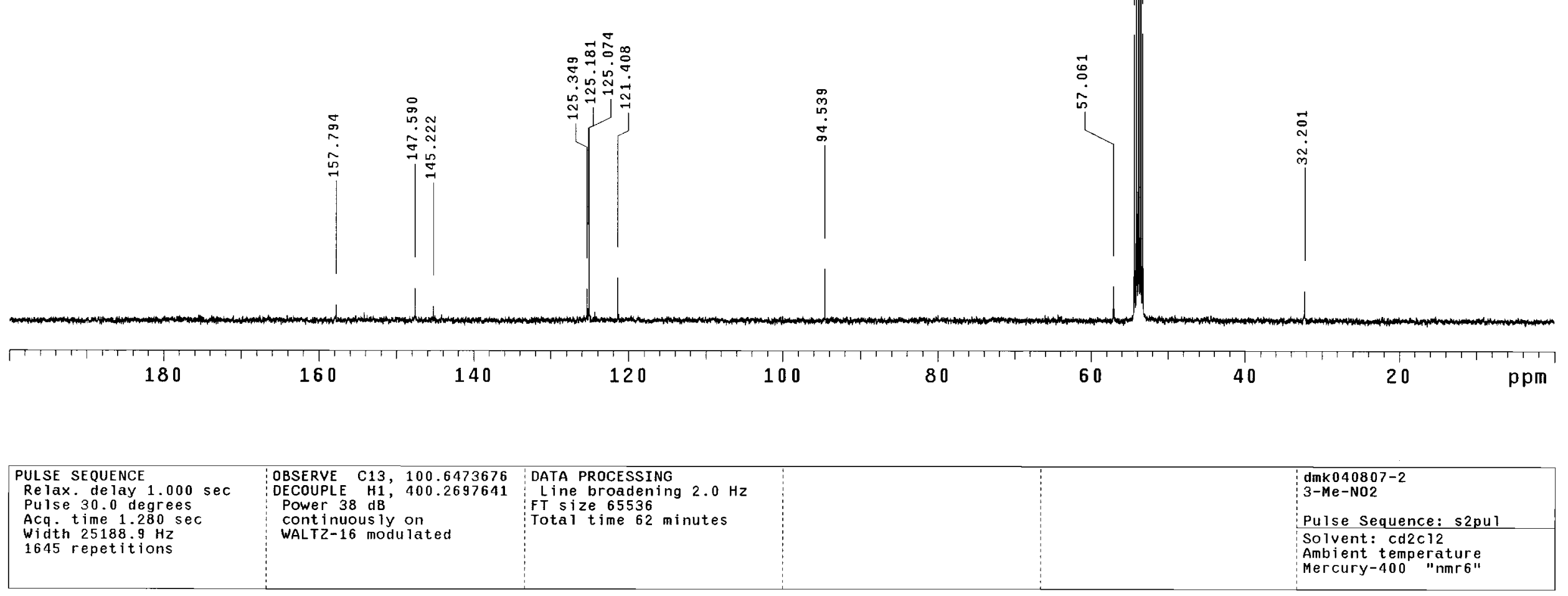


${ }_{\mathrm{O}}^{\mathrm{O}}=\mathrm{N}-\mathrm{N}=\mathrm{N}-\mathrm{NO}_{2}$

$3-\mathrm{OCH} 3-\mathrm{NO} 2$

S84

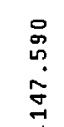

|

|

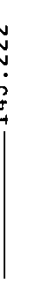

If 148 140 il 138

PULSE SEQUENCE
Relax. delay $1.000 \mathrm{sec}$ Relax. delay 1.000
Pulse 30.0 degrees

Acq time $1.280 \mathrm{sec}$ 1648 repetitions
OBSERVE C13, 100.6473676

POWPr 38 dB

cont inuous iy on

WAL TZ-16 modulated
DATA PROCESSING

Line broadening $2.0 \mathrm{~Hz}$

FT size 65536
Total time 62 minutes dmk040807-2

Pulse Sequence: s2pul

Solvent: cd2c12

Ambient temperature 


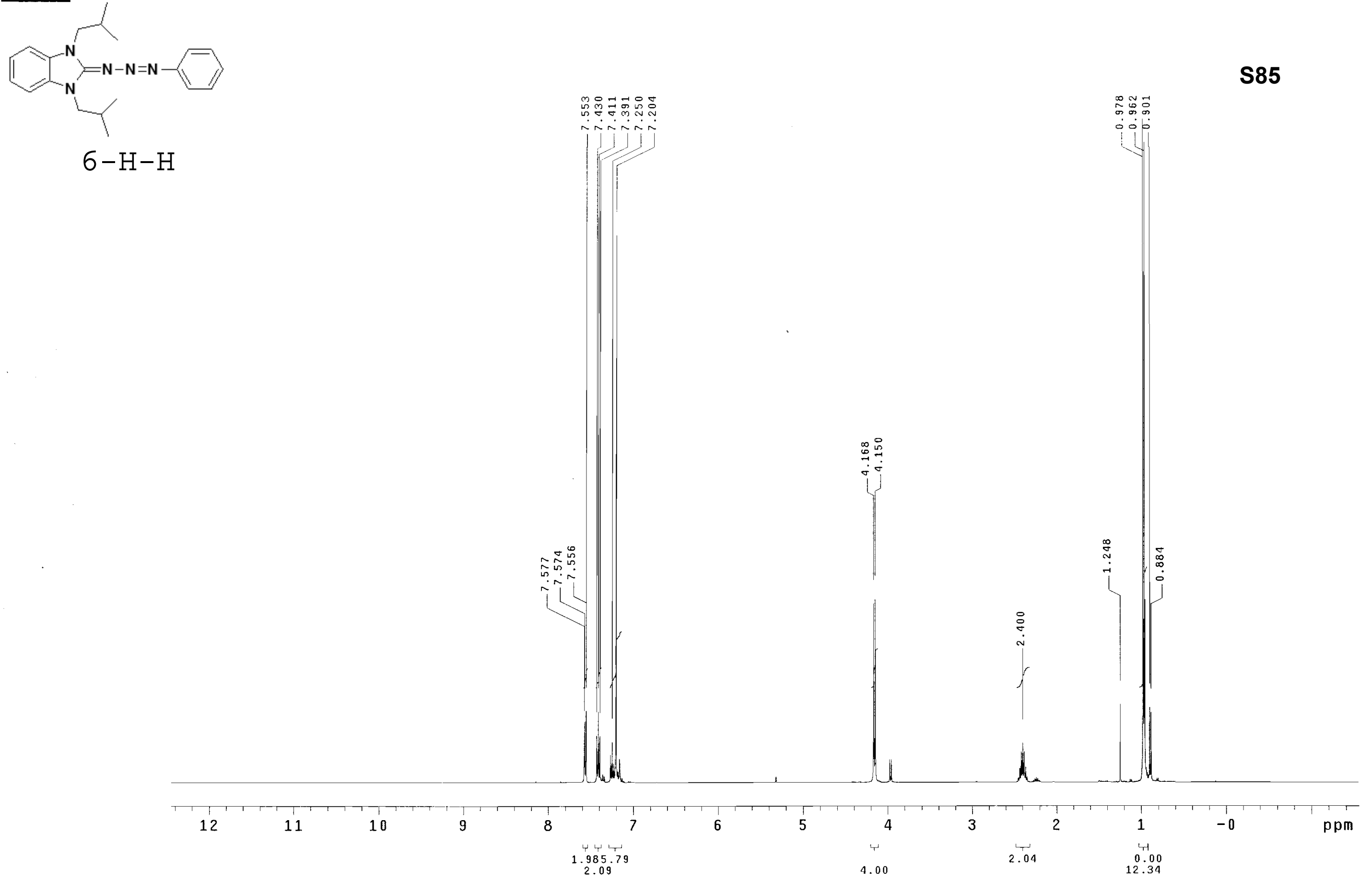

\begin{abstract}
PULSE SEQUENCE
Re lax. de lay $1.000 \mathrm{sec}$ Relax. delay 1.000 s
Pulse 32.7 degrees
Acq Acq time 2.856 sec 3 repetitions
\end{abstract}

OBSERVE

H1, 400.2677522 DATA PROCESSING Line broadening $0.1 \mathrm{~Hz}$ FT size 32768
Total time 1 minute dmk102306-1 iBu-H
triazene

Pulse Sequence: s2pur Solvent: $\mathrm{Cd}_{2} \mathrm{cl} 2$ Ambient temperature
Mercury-400 "nmr6" 


$$
6-\mathrm{H}-\mathrm{H}
$$

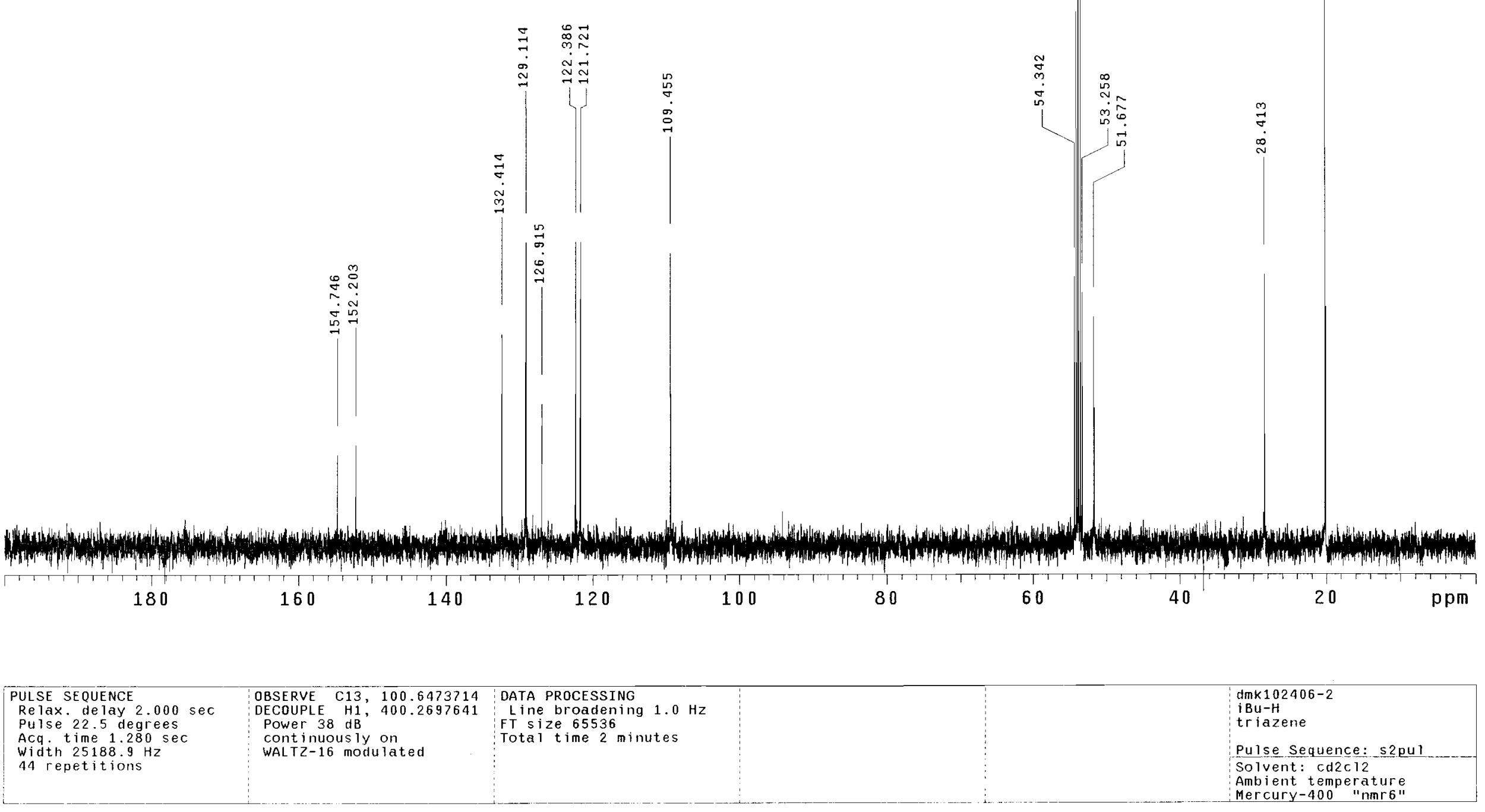



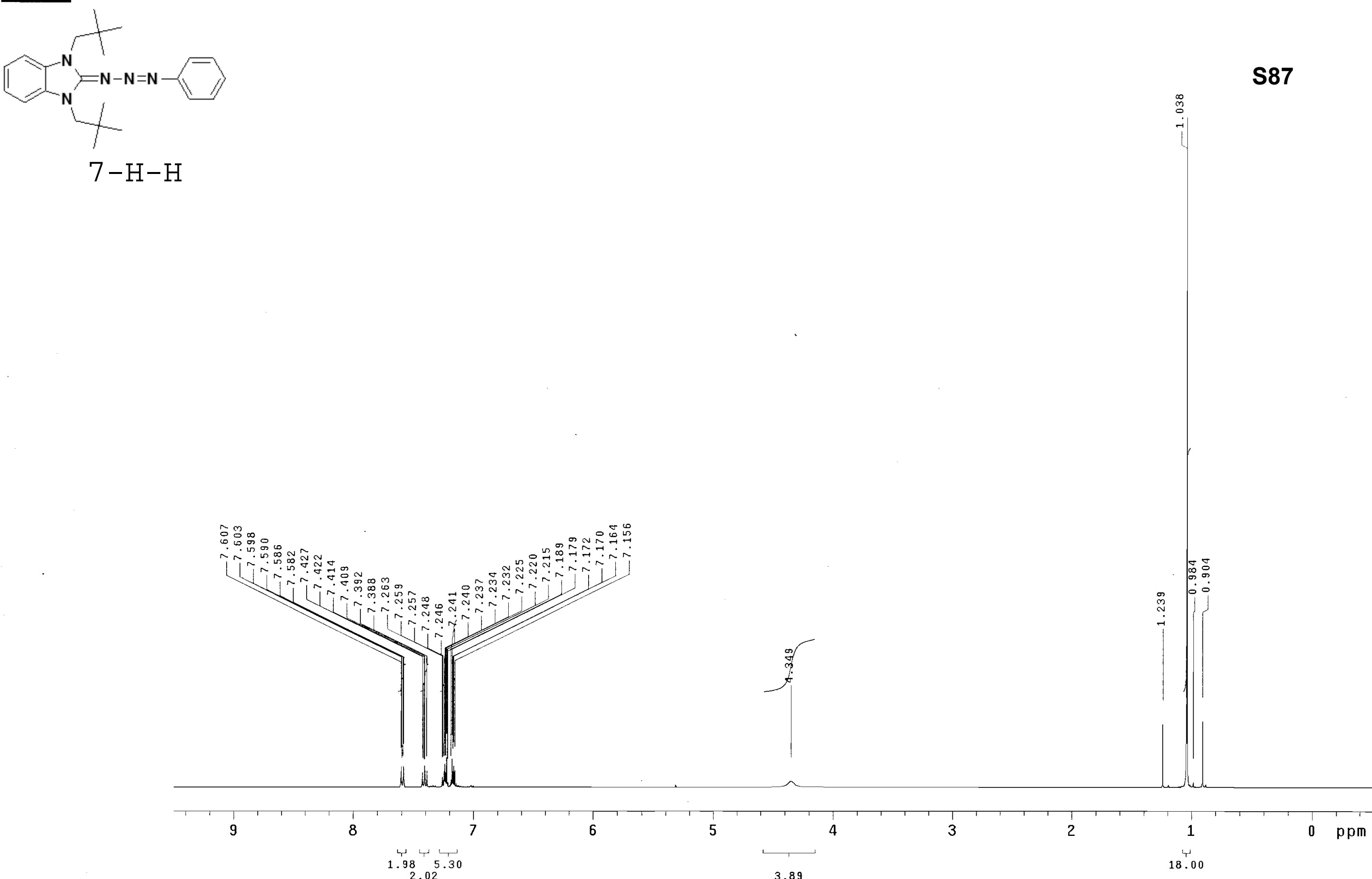

PULSE SEQUENCE

OBSERVE H1, 400.2677533 DATA PROCESSING

Relax. de lay 1.000 sec

Acq. time $2.856 \mathrm{sec}$

Width $5602.2 \mathrm{~Hz}$
4 repetitions

Line broadening $0.1 \mathrm{~Hz}$

Total time 1 minute

dmk102306-1

neopenty 1

Pulse Sequence: s2pul

Solvent: cd2cl2

Ambient temperature 

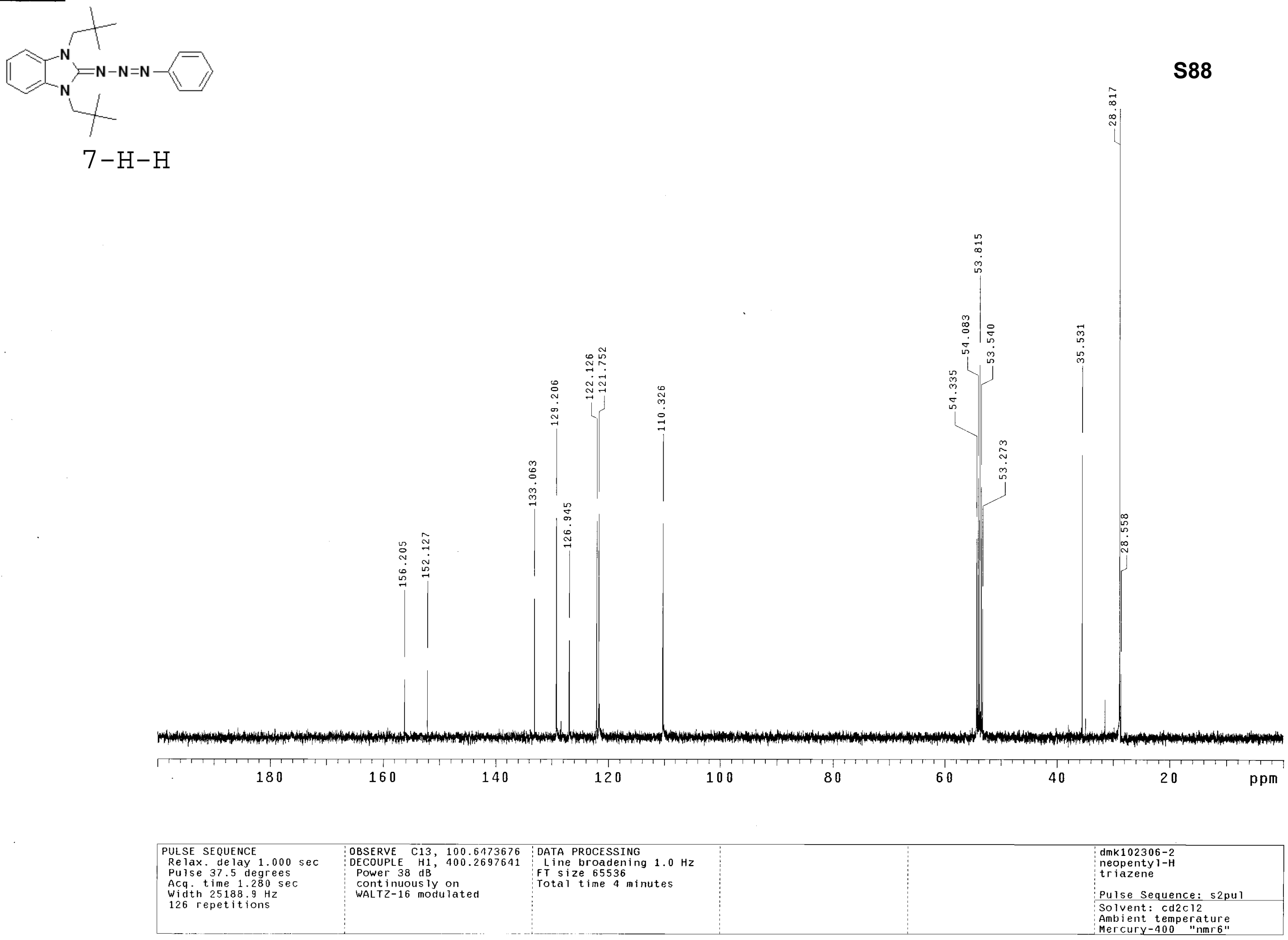


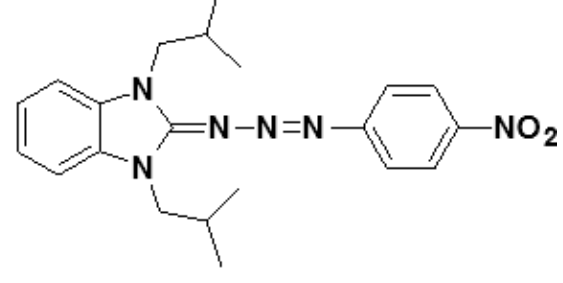

$$
6-\mathrm{H}-\mathrm{NO} 2
$$

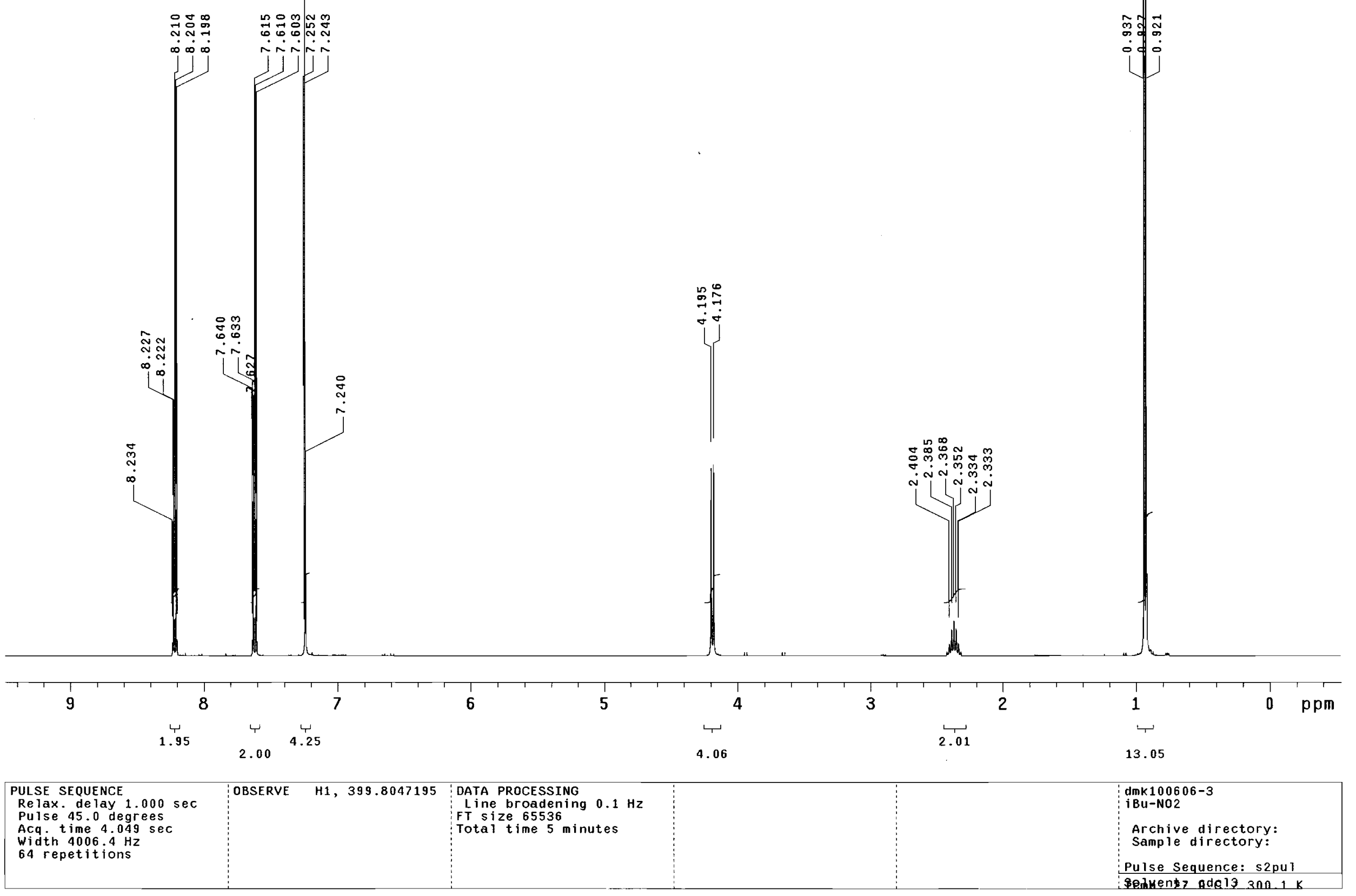




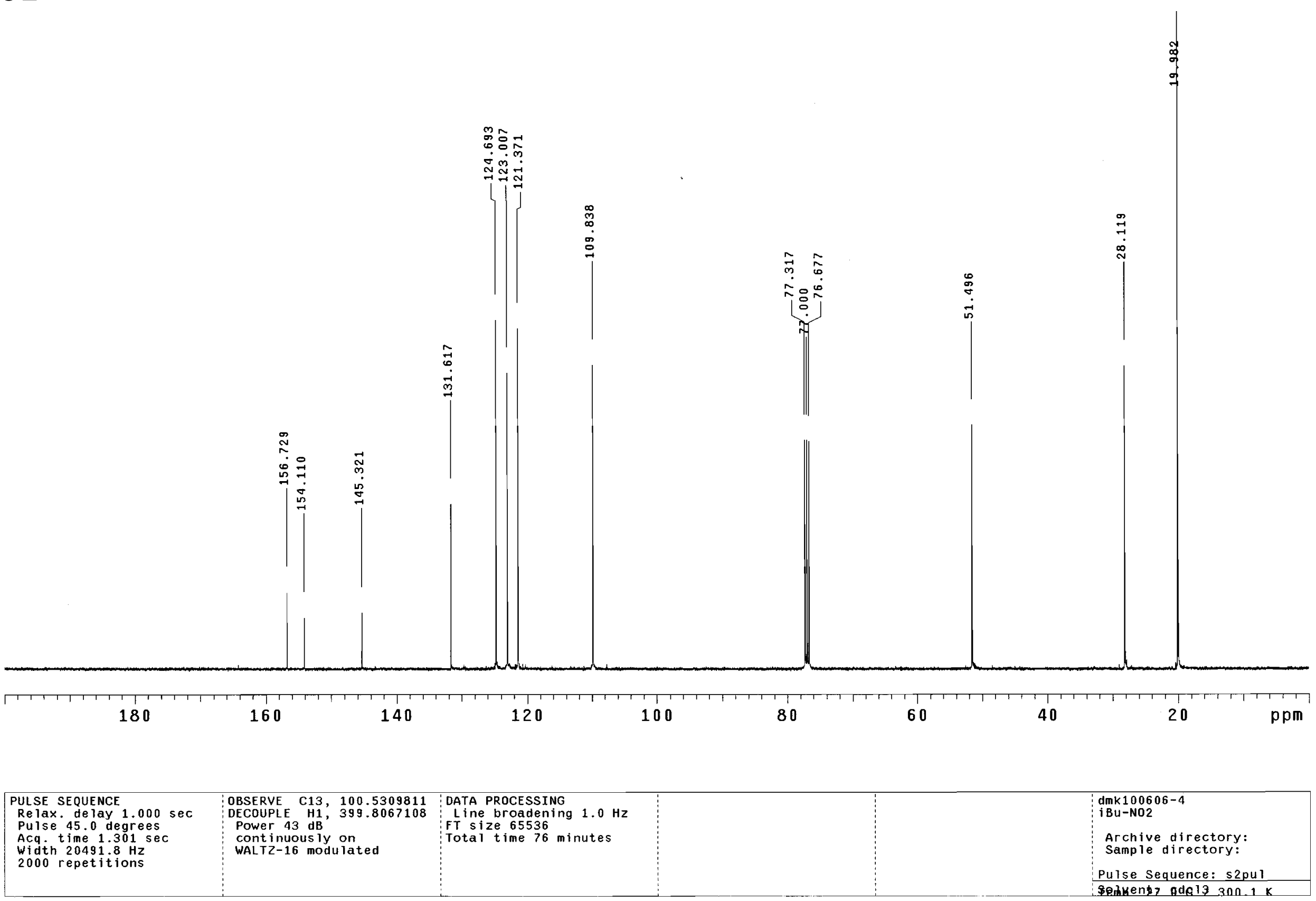




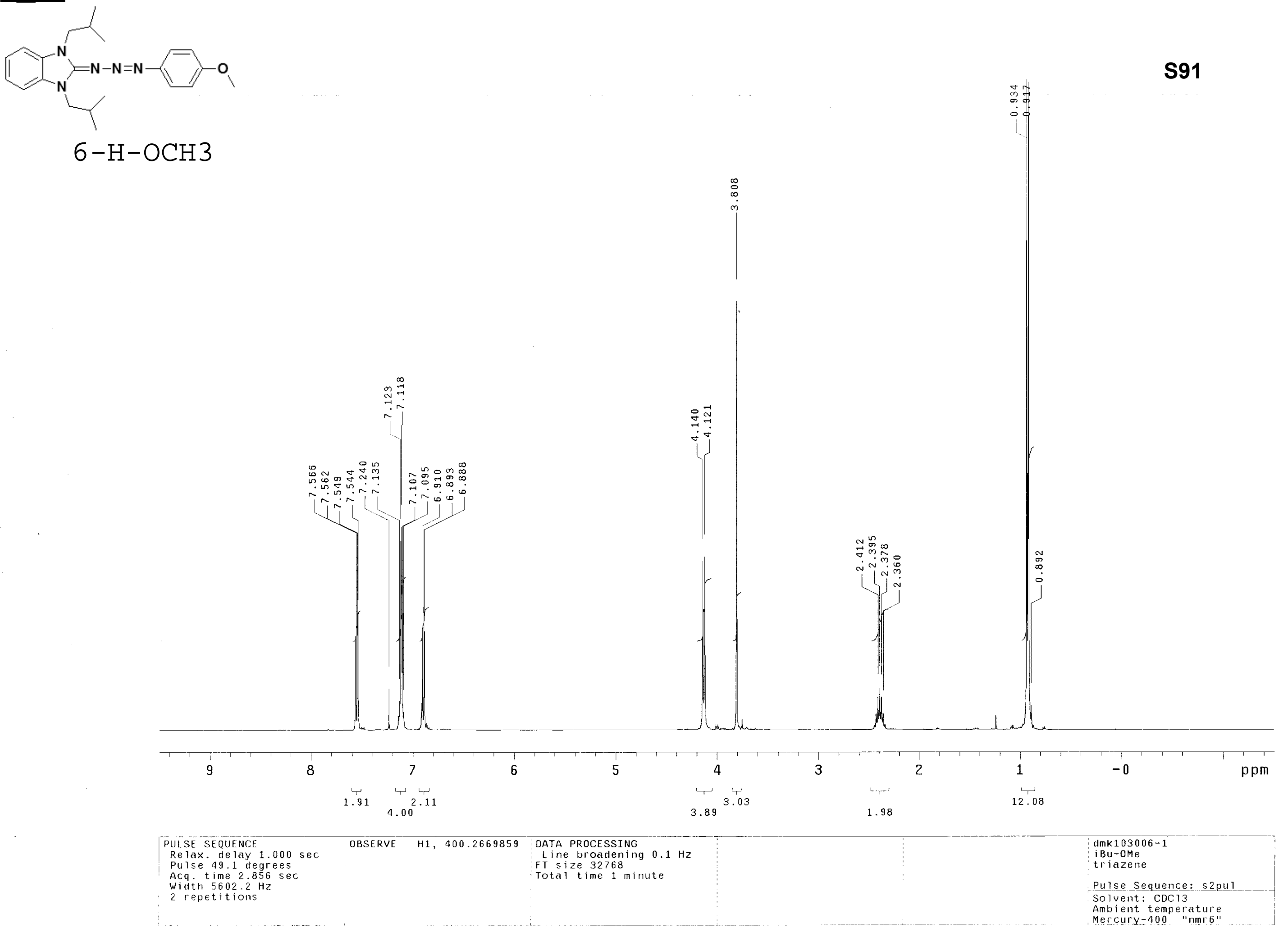




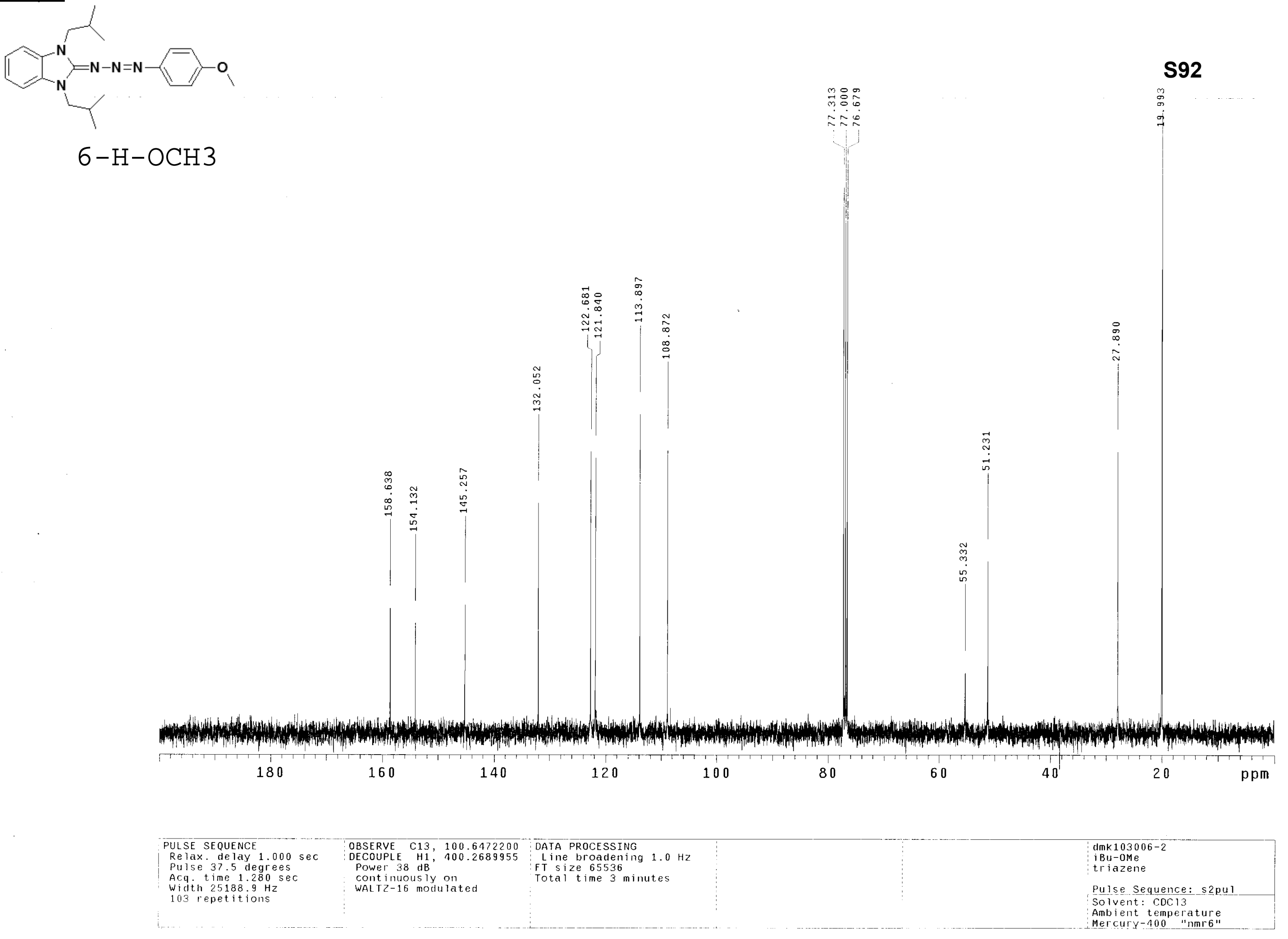




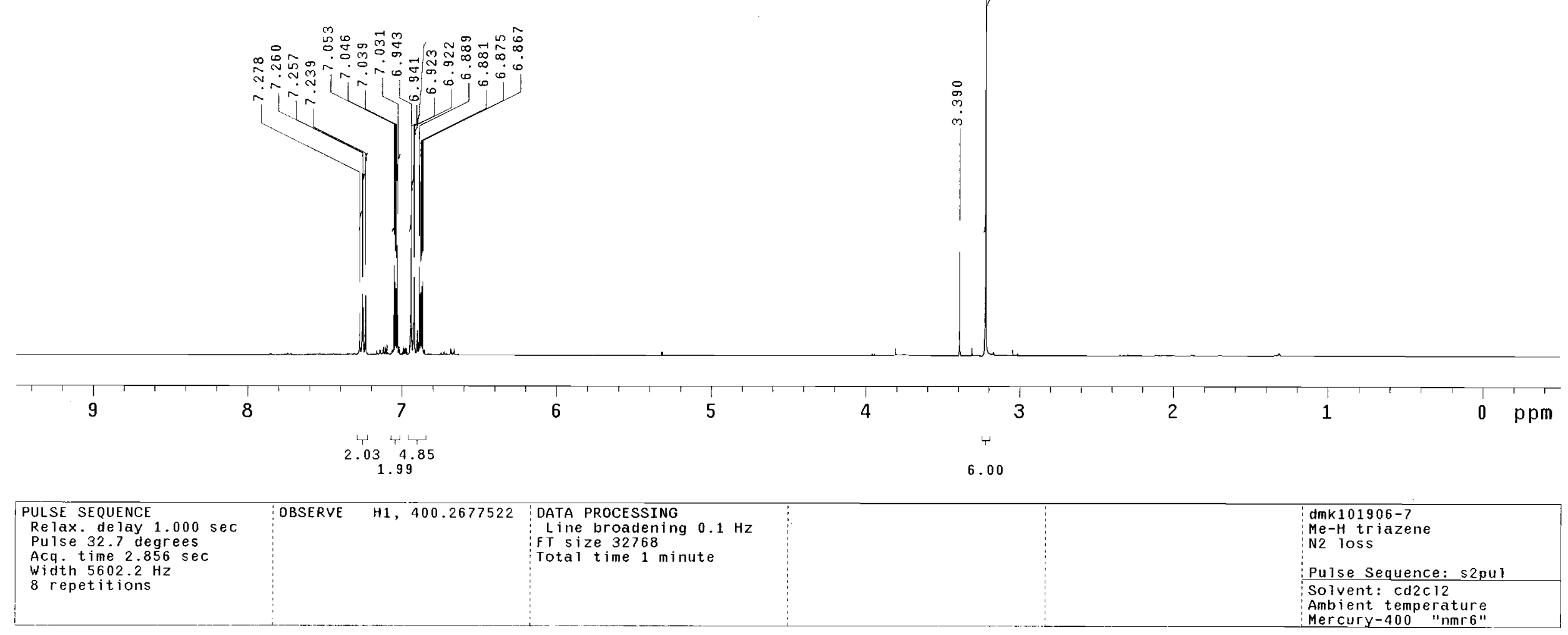




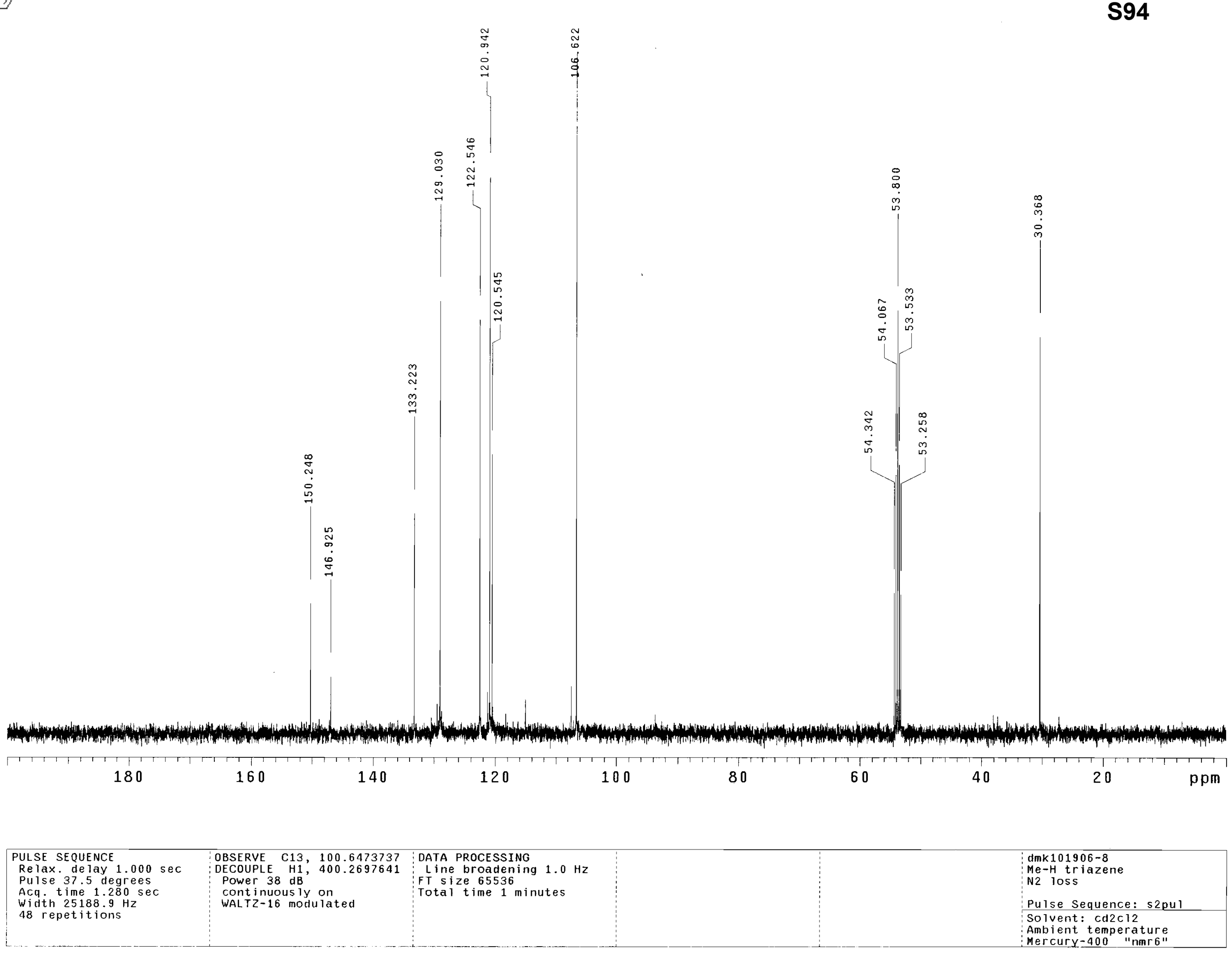



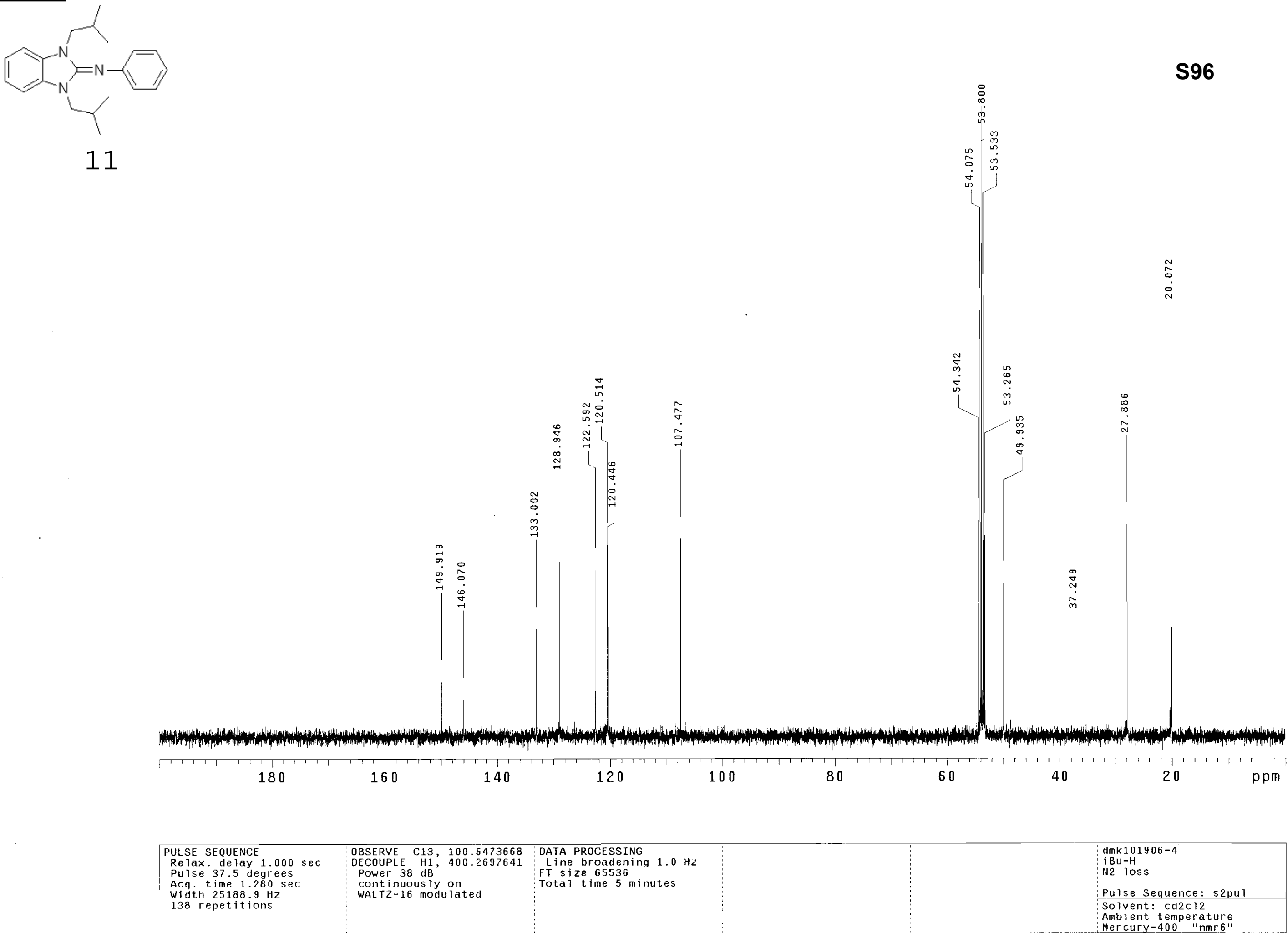

00

80

60

40

20

ppm

\begin{tabular}{|c|c|c|}
\hline $\begin{array}{l}\text { PULSE SEQUENCE } \\
\text { Relax. delay } 1.000 \mathrm{sec} \\
\text { Pulse } 37.5 \mathrm{degrees} \\
\text { ACq. time } 1.280 \mathrm{sec} \\
\text { Width } 25188.9 \mathrm{~Hz} \\
\text { 138 renetitions }\end{array}$ & $\begin{array}{l}\text { OBSERVE C13, } 100.6473668 \\
\text { DECOUPLE H1, } 400.2697641 \\
\text { POwer } 38 \text { dB } \\
\text { COnt inuous Iy on } \\
\text { WALTZ-16 modulated }\end{array}$ & $\begin{array}{l}\text { DATA PROCESSING } \\
\text { Line broadening } 1.0 \mathrm{~Hz} \\
\text { FT size } 65536 \\
\text { Total time } 5 \text { minutes }\end{array}$ \\
\hline
\end{tabular}

dmk101906-4 i Bu-H
N2 loss

Pulse Sequence: s2pul Solvent: $\mathrm{cd} 2 \mathrm{Cl} 2 \mathrm{2}$ Mercury-400 "nmrs" 

